\title{
A GLUING FORMULA FOR THE ANALYTIC TORSION ON HYPERBOLIC MANIFOLDS WITH CUSPS
}

\author{
JONATHAN PFAFF
}

\begin{abstract}
For an odd-dimensional oriented hyperbolic manifold with cusps and strongly acyclic coefficient systems we define the Reidemeister torsion of the Borel-Serre compactification of the manifold using bases of cohomology classes defined via Eisenstein series by the method of Harder. In the main result of this paper we relate this combinatorial torsion to the regularized analytic torsion. Together with results on the asymptotic behaviour of the regularized analytic torsion, established previously, this should have applications to study the growth of torsion in the cohomology of arithmetic groups. Our main result is established via a gluing formula and here our approach is heavily inspired by a recent paper of Lesch.
\end{abstract}

\section{Contents}

1. Introduction

2. Preliminaries

3. Homogeneous and flat vector bundles

4. The De Rham complex on the cusp 15

5. Lifts of differential forms from the boundary to the cusp 16

6. Closed forms on the cusp 18

7. Eisenstein series 21

8. Bases of cohomology classes on certain flat bundles using Eisenstein series 23

9. The Reidemeister torsion with respect to Eisenstein cohomology classes 28

10. Regularized and relative traces and torsions 31

11. Some properties of the heat kernels on locally homogeneous vector bundles 40

12. The variation of the De Rham complex after Vishik and Lesch 44

13. The heat kernel of the perturbed Laplacian 48

14. The first version of the gluing formula

15. The second version of the gluing formula 60

16. The contribution of the cohomology to the gluing formula $\quad 67$

\begin{tabular}{ll} 
References & 69 \\
\hline
\end{tabular}

\section{INTRODUCTION}

The aim of the present paper is to study the relation between analytic and combinatorial torsion on odd-dimensional hyperbolic manifolds with cusps with coefficients in strongly 
acyclic coefficient systems by establishing a gluing formula. Our approach to the gluing formula is heavily inspired by a recent paper of Lesch [Le], whose work was built on earlier work of Vishik [Vi].

Let us describe the situation we consider in more detail. Let $X$ be an odd-dimensional hyperbolic manifold of the form $X=\Gamma \backslash \mathbb{H}^{d}, \Gamma$ a discrete, torsion-free subgroup of $\operatorname{SO}^{0}(d, 1)$ or $\operatorname{Spin}(d, 1)$ such that $X$ is of finite volume but not compact. Then $X$ is a manifold with cusps. More precisely, there exist finitely many closed manifolds $T_{i}$ with Riemannian metrics $g_{i}$ such that for $Y \geq Y_{0}, Y_{0}$ sufficiently large, $X$ can be decomposed into a compact smooth manifold with boundary $X(Y)$, whose boundary $\partial X(Y)$ is the disjoint union of the $T_{i}$, and the disjoint union of finitely many cusp pieces $F_{i, X}(Y):=[Y, \infty) \times T_{i}$, glued together with $X(Y)$ along the $T_{i}$. Moreover, the hyperbolic metric $g$ of $X$ restricted to each cusp $F_{i, X}(Y)$ equals the warped product metric $y^{-2}\left(d y^{2}+g_{i}\right)$. Over $X$ we consider a flat vector bundle $E_{\rho}$ induced by the restriction to $\Gamma$ of a finite-dimensional irreducible representation $\rho$ of the group $\operatorname{SO}^{0}(d, 1)$ or $\operatorname{Spin}(d, 1)$. We assume that $\rho$ is not invariant under the standard Cartan involution $\theta$ of $G$. Such representations exist in abundance and following Bergeron and Venkatesh [BV], we call the flat bundle $E_{\rho}$ strongly acyclic. The bundle $E_{\rho}$ is unimodular and moreover possesses a canonical metric.

Since $X$ is complete, the underlying flat $E_{\rho}$-valued Hodge-Laplacians on $X$ are essentially selfadjoint. However, these operators have a large continuous spectrum and therefore, the corresponding heat operators are not trace class. To overcome this problem, we work with the so called regularized trace [Par], [MP2], [MP5] whose definition is inspired by ideas of Melrose $[\mathrm{Me}$. More precisely, the integral of the heat kernels over the sets $X(Y)$ has a well defined asymptotic expansion in $Y$ and one defines the regularized trace as the constant term in this expansion. In this way, one can define the regularized analytic torsion $T_{\text {reg }}\left(X ; E_{\rho}\right)$. For more details we refer to section 10 .

For compact smooth manifolds the analytic torsion, introduced by Ray and Singer [RS], equals the corresponding Reidemeister torsion. For the case of closed manifolds and unitary representations of the fundamental group, this was proved independently by Cheeger [C1] and Müller [Mü1]. The extension to unimodular representations is due to Müller [Mü4 and has also been obtained independently and in a more general setting by Bismut and Zhang [BZ]. Several different proofs and also several important generalizations of this theorem to the case of manifolds with boundary and the equivariant case had been obtained in the sequel. Without aiming at completeness, let us just mention the work of Hassell [Has], Lück [Lu], Vishik [Vi], Brüning and Ma [BM1] [BM2] and Lott and Rothenberg [LR] here.

In the present case, the manifold $X$ does have a natural compactification $\bar{X}$. More precisely, $\bar{X}$ is by definition the diffeomorphism class of the manifold $X(Y)$ as above. Thus, $\bar{X}$ is a compact smooth manifold with boundary which is homotopy equivalent to $X$. However, the manifold $\bar{X}$ does not have any canonical Riemannian structure. More precisely, the manifolds $X\left(Y_{1}\right)$ and $X\left(Y_{2}\right)$ respectively the corresponding flat bundles are not isometric for different values $Y_{1}$ and $Y_{2}$. Consequently, in particular since the bundle $E_{\rho}$ restricted to these manifolds has non-vanishing De Rham cohomology in our situations, the 
analytic torsions $T\left(X\left(Y_{1}\right) ; E_{\rho}\right)$ and $T\left(X\left(Y_{2}\right) ; E_{\rho}\right)$, taken with respect to absolute boundary conditions, differ. For this reason we study the regularized analytic torsion, which is a spectral invariant of the complete hyperbolic manifold $X$. Nevertheless, the CheegerMüller Theorem for manifolds with boundary, due to Lück [Lu], Vishik [Vi] and Brüning and Ma [BM1, BM2], will play an important role in our article.

On the combinatorial side, the situation is similar. If one wants to define the Reidemeister torsion of $\bar{X}$ as a positive real number and not just as a non-zero element in the determinant line of the cohomology $H^{*}\left(\bar{X} ; E_{\rho}\right)$, one needs to fix a metric on this determinant line. However, if one takes the metrics on the cohomology groups $H^{*}\left(X\left(Y_{1}\right) ; E_{\rho}\right)$ and $H^{*}\left(X\left(Y_{2}\right) ; E_{\rho}\right), Y_{2}>Y_{1}$, defined by the Hodge De Rham isomorphism with respect to absolute boundary conditions, then the canonical inclusion $X\left(Y_{1}\right) \hookrightarrow X\left(Y_{2}\right)$ induces an isomorphism, but not an isometry on cohomology. Therefore, one does not obtain a well defined Reidemeister torsion which is independent of the truncation parameter.

To overcome this problem, we will work with bases in the cohomology of $\bar{X}$ with coefficients in $E_{\rho}$ using the constructions of Harder [Ha]. The condition $\rho \neq \rho_{\theta}$ implies that the $L^{2}$-cohomology of $X$ with coefficients in $E_{\rho}$ vanishes. Therefore, in the present situation the cohomology $H^{*}\left(\bar{X} ; E_{\rho}\right)$ is determined completely by its image in the cohomology $H^{*}\left(\partial \bar{X} ; E_{\rho}\right)$ of the boundary. The cohomology of the boundary is in turn understood: By a result of van Est [ $\mathrm{vE}$, for each boundary component it equals the corresponding twisted cohomology of a nilpotent Lie algebra $\mathfrak{n}$ associated to that component; the latter cohomology has been evaluated by Kostant [Ko. The idea of Harder [Ha now was to lift cohomology classes from the boundary to cohomology classes of $X$ using the method of Eisenstein series and therefore to obtain a certain inverse of the restriction map in cohomology. His approach was developed further by Schwermer Sch. In the present context, this construction gives bases of all cohomology groups $H^{*}\left(X ; E_{\rho}\right) \cong H^{*}\left(\bar{X} ; E_{\rho}\right)$. Moreover, the resulting Reidemeister torsion does not depend on the particular choices made in the construction of such bases. Therefore, one obtains a well defined Reidemeister torsion $\tau_{E i s}\left(\bar{X} ; E_{\rho}\right) \in(0, \infty)$. We will pursue these constructions in detail in sections 4 to 8. At this point we remark that in their recent book [CV], Calegari and Venkatesh also used Eisenstein series to give a canonical definition of Reidemeister torsion in a related 3-dimensional context.

To state our main theorem, we need to introduce some more notation. Firstly, if $\partial \bar{X}_{i}$ is a boundary component of $\bar{X}$ and if $A$ is the split component of the $\Gamma$-cuspidal parabolic subgroup $P$ of $G$, implicit in the choice of $\partial \bar{X}_{i}$, then each cohomology group $H^{k}\left(\partial \bar{X}_{i} ; E_{\rho}\right)$ carries a natural action of $A$. In our real rank one case, one has $A \cong \mathbb{R}$. By Kostant's theorem, the corresponding weights $\lambda_{\rho, k} \in \mathfrak{a}^{*} \cong \mathbb{R}$ of this action can be computed explicitly in terms of the highest weight of $\rho$, see (6.6), (6.7). Next, in the notation of the beginning of this section, we let $F_{X}$ be the union of the cusps $[1, \infty) \times T_{i}$ at height one, equipped with the warped product metric $y^{-2}\left(d y^{2}+g_{i}\right)$. We point out that we do not require that $F_{X}$ is actually a subset of $X$, i.e. the cusps may be separated only at a height which is greater than 1. Nevertheless, $F_{X}$ is unambiguously defined. We let $T_{\mathrm{reg}}\left(F_{X}, \partial F_{X} ; E_{\rho}\right)$ 
be the regularized analytic torsion of the cusp with relative boundary conditions which is defined in the same way as the regularized analytic torsion of $X$. The last ingredient of our theorem is the Brüning-Ma anomaly formula for the manifold $F_{X}$. Although $F_{X}$ is not compact, its boundary is compact and since in our present unimodular case the anomaly is completely local in a neighbourhood of the boundary, it can be defined by integrating the same secondary characteristic form as in [BM1], [BM2] over $\partial F_{X}$, see section [15. By the explicit computations of Brüning and Ma [BM1], for the present case the resulting integral equals $c(n) \operatorname{rk}\left(E_{\rho}\right) \operatorname{vol}\left(\partial F_{X}\right)$, where $c(n) \neq 0$ is a constant, defined in (15.10), which depends only on the dimension $d=2 n+1$ of $X$.

We further make an assumption (2.11) on $\Gamma$ which holds for example if $\Gamma$ is "neat" in the sense of Borel [Bo1]. This will be discsussed further in section 2.7. This assumption is not essential for our approach and can certainly be dropped. If it is not satisfied, the cohomology of the boundary is a bit more complicated and one has to twist with the additional actions of certain finite groups. However, since for example in the arithmetic case, intersecting with a fixed principal congruence subgroup of sufficiently high level assumption (2.11) is satisfied, i.e. (2.11) holds by passing to finite coverings, we shall assume it for convenience from now on.

The main result of this article is now the following theorem.

Theorem 1.1. For every $\rho \in \operatorname{Rep}(G), \rho \neq \rho_{\theta}$ one has

$$
\begin{aligned}
\log \tau_{E i s}\left(\bar{X} ; E_{\rho}\right)= & \log T_{\text {reg }}\left(X ; E_{\rho}\right)-\log T_{\text {reg }}\left(F_{X}, \partial F_{X} ; E_{\rho}\right)+c(n) \operatorname{rk} E_{\rho} \operatorname{vol}\left(\partial F_{X}\right) \\
& -\frac{1}{4} \sum_{k=0}^{d-1}(-1)^{k} \log \left(\left|\lambda_{\rho, k}\right|\right) \cdot \operatorname{dim} H^{k}\left(\partial \bar{X} ; E_{\rho}\right) .
\end{aligned}
$$

We remark that, as one might expect from the Cheeger-Müller Theorems for manifolds with boundary mentioned above and from Lesch's main result, the Euler characteristic of $\bar{X}$ resp. $\partial \bar{X}$ occurs during our proof several times. However, it is always zero in our case since $\partial \bar{X}$ is a finite disjoint union of tori and therefore it doesn't appear in our main result. Next we remark that if $d=2 n+1$ and if $\kappa(X)$ is the number of cusps of $X$, then one has $\operatorname{dim} H^{k}\left(\partial \bar{X} ; E_{\rho}\right)=\kappa(X) \operatorname{dim} \sigma_{\rho, k}$ for $k \neq n$ and $\operatorname{dim} H^{n}\left(\partial \bar{X} ; E_{\rho}\right)=2 \kappa(X) \operatorname{dim} \sigma_{\rho, n}$, where $\sigma_{\rho, l}$ are representations of $M \cong \operatorname{SO}(d-1)$ or $M \cong \operatorname{Spin}(d-1)$ whose highest weight can be computed explicitly from the highest weight of $\rho$ by Kostant's theorem, see (6.5), (6.7). Therefore, the second line in our main theorem is explicit by Weyl's dimension formula.

We shall now describe our approach to prove our main result. We remark in the beginning that we do not use any computational properties of the involved regularized traces. In particular, we do not use the Selberg trace formula. Instead, we study the problem directly via the gluing formula.

We have the following gluing situation: For a fixed $Y_{1}>Y_{0}$ we consider the decomposition $X=X^{+} \cup X^{-}$, where $X^{+}:=X\left(Y_{1}\right)$ and $X^{-}:=F_{X}\left(Y_{1}\right)$. We firstly replace the metrics on $X$ and $E_{\rho}$ by metrics which are of product structure in a neighbourhood of $\partial X^{+}$. Then we will study a variation of the $E_{\rho}$-valued De Rham complex, which is due to Lesch [Le], based 
on earlier work of Vishik [Vi]. This variation interpolates smoothly between the $E_{\rho}$-valued De Rham complex of $X$ and the direct sum of the De Rham complex of $X^{+}$with absolute boundary conditions and the De Rham complex of $X^{-}$with relative boundary conditions. The goal is now to study the behaviour of the torsion under this variation. Here, in order to avoid interchanges of limits, instead of using the regularized torsion, we will work with the relative torsion that will be defined in section 10. In the end, one can pass back to the regularized torsion since the difference between both torsions is explicit.

In our understanding, Lesch's approach [Le] to study the behaviour of the torsion under the variation can be divided into two parts. Firstly, one establishes a variation formula for the weighted sum of the perturbed heat traces for each fixed time [Le, Theorem 5.3]. This variation is local. Therefore, in our case we will establish it via an approximation of our variation through variations on closed manifolds, to which we can apply Lesch's formula. The result will be Proposition 14.3. Here we recall that in our situation the Laplace operators have a large continuous spectrum, which is the main difference to Lesch's paper, who assumes that the spectrum is discrete. Therefore, in order to generalize Lesch's variation formula in Proposition 14.3, we need to pursue a detailed analysis of the underyling heat kernels, which will be carried out mostly in sections 11, 13, and 14.

However, by the presence of large time values of the heat operators, the analytic torsion is not a local invariant. Due to this fact, a global term in the variation formula of Lesch appears, namely the torsion of the long exact cohomology sequence [Le, section 5.2, section 5.3]. We recall that in our situation the Laplace operators of the underlying Hilbert complexes have a large continuous spectrum. Nevertheless, due to our assumption on the bundle $E_{\rho}$, these complexes are still Fredholm complexes in the sense of Brüning and Lesch [BL]. Therefore, as proved by these authors [BL], they still satisfy the so called strong Hodge decomposition theorem. This property is sufficient to generalize Lesch's argument of [Le, section 5.2, section 5.3] and to establish the full gluing formula, Theorem 14.7, for the analytic torsion with respect to the perturbed metrics.

In the next step, we have to pass back from the perturbed metrics to the original metrics. Here, we will apply the anomaly formula of Brüning and Ma [BM1], [BM2]. This is possible since in our unimodular case the formula of Brüning and $\mathrm{Ma}$ is local at the boundary of the underlying manifolds. Then, via a gluing formula for the cusps $F_{X}\left(Y_{1}\right)$, we get rid of the truncation parameter by passing from $F_{X}\left(Y_{1}\right)$ to $F_{X}$. Moreover, by the Cheeger-Müller theorem for manifolds with boundary and the anomaly formula, we can relate the analytic torsion of the compact part $X^{+}$to the Reidemeister torsion of $X^{+}$. However, we need then to change the bases in the cohomology and to pass to the Eisenstein cohomology classes in order to make this torsion on $X^{+}$equal to the torsion $\tau_{E i s}\left(\bar{X} ; E_{\rho}\right)$. Also, we need to compute the corresponding torsion of the long exact cohomology sequence explicitly. For this purpose, in section 6 and section [16 we will construct explicitly closed harmonic forms in the cusp which satisfy relative boundary conditions by adapting Harder's arguments. Then we will obtain the last line in Theorem 1.1 as the contribution of the cohomology.

In our understanding, a very important example of a situation considered by Lesch would be a compact manifold $X$ with a conical singularity such that in the gluing formula $X^{+}$ 
is a smooth manifold with boundary and such that $X^{-}=N$ contains the cone point, see the introduction of [Le]. By the work of Cheeger [C2], on such manifolds the flat Hodge Laplacians, resp. their suitably defined selfadjoint extensions, have discrete spectrum and moreover the analytic torsion can be defined in exactly the same way as on closed manifolds. One now hopes to compare this analytic torsion to the combinatorial torsion of a suitable finite-dimensional complex on $X$. Since the combinatorial torsion of a chain complex satisfies a gluing formula which is exactly of the same type as the gluing formula established by Lesch [Le, Theorem 6.1], see [Le, Appendix A], by Lesch's main result and by the Cheeger-Müller theorems for smooth manifolds with boundary [Lu], [Vi] [BM2], the conjectured equality of analytic and combinatorial torsion on the singular manifold $X$ would be proved if it was proved on $N$.

Our gluing situation is however different. The point is that the combinatorial torsion we are interested in, in particular due to possible arithmetic applications sketched below, is the combinatorial torsion $\tau_{\text {Eis }}\left(\bar{X} ; E_{\rho}\right)$ of the manifold $\bar{X}$. This torsion can be computed using the manifold $X^{+}$which appears in our gluing situation. Therefore, one does not have to find a combinatorial analog for the regularized analytic torsion of the cusps $X^{-}$ with relative boundary conditions. Instead, we think that using a separation of variables technique [M̈̈2], one could obtain an explicit formula for the regularized analytic torsion of the cusps in terms of special values of spectral zeta functions on its boundary components, which are just flat tori. A similar result for the case of a conical singularity was obtained by Vertman [Ve]. What we have to study in our paper, in contrast to Lesch's situation, where this term has a combinatorial analog, is however the contribution of the torsion of the long exact cohomology sequence to the gluing formula. The result will be the second line in our Theorem 1.1.

We remark that in our earlier paper [Pf2], a specific comparison between certain regularized analytic torsions and certain Reidemeister torsions was obtained for the non-compact, finite-volume 3-dimensional hyperbolic case. The proof used a completely different method and was based on an explicit evaluation of special values of Ruelle zeta functions and on a result of Menal-Ferrer and Porti [MePo]. The method of [Pf2] does not generalize to higher dimensions and not even to general strongly acyclic coefficient systems in the 3-dimensional case. Also, the result of [Pf2] only holds for the quotients of two torsions associated to different representations of the fundamental group and it does not use Eisenstein cohomology classes as a basis.

Next we would like to point out that the assumption $\rho \neq \rho_{\theta}$ is essential for our method. If this assumption is not satisfied, then the $L^{2}$-cohomology with coefficients in $E_{\rho}$ is infinitedimensional in the middle dimension by a result of Borel and Casselmann [BC, 4.6 (a)]. Equivalently [BL, zero belongs to the essential spectrum of the corresponding flat Hodge Laplace operator. Therefore, to treat such a case is far beyond the scope of the present paper. We remark that even in the compact locally symmetric case the analytic torsion becomes extremely more complicated if $\rho$ is no longer strongly acyclic, essentially due to the same problem of small eigenvalues. In particular, an analog of the main result of 
Bergeron and Venkatesh [BV] on the asymptotic behaviour of analytic torsion is, as far as we know, not established for such a situation even in the compact case.

Mainly due to this particular choice of our flat bundle we also restrict to the true locally symmetric case and do not work with more general manifolds with cusps in the sense of Müller [M̈̈3], or, more precisely, with Riemannian manifolds that have the structure of an odd-dimensional hyperbolic manifold with cusps only outside some compact set. Our method would work here if we were given a flat bundle whose $L^{2}$-cohomology would vanish or at least be finite-dimensional; the theory of Eisenstein series, which we use to define bases in the cohomology, can be extended to such manifolds [Mü3, chapter 8]. However, while in the locally symmetric case bundles of the required form exist in abundance, we do not know whether there is a construction of such bundles on more general manifolds with cusps.

We also remark that at least in the compact locally symmetric case the analytic torsion with coefficients in a flat bundle induced by a finite-dimensional representation of $G$ is always equal to 1 if the dimension of the manifold is even, [MS], [BMZ], [MP3]. Moreover, the present odd-dimensional hyperbolic case is exactly the case corresponding to an irreducible symmetric space of real rank one which is odd dimesional. A treatment of a suitable analog of the analytic torsion in the non-compact higher rank case is again far beyond the scope of the present article.

We finally outline how our main result could be applied to study the growth of torsion in the cohomology of certain arithmetic groups with coefficients in certain integral local systems. Here, starting with the paper [BV] of Bergeron and Venkatesh, the analytic torsion arose in the following context. If the locally symmetric space is associated to an arithmetic group $\Gamma$ and if the flat bundle is associated to a representation of this group on a free finite rank $\mathbb{Z}$-module $L$, then, as observed by Bergeron and Venkatesh [BV], see also [C1], the Reidemeister torsion encodes information about the sizes of the torsion part in the cohomology of $\Gamma$ with coefficients in $L$. This relation continues to hold for non-cocompact $\Gamma$ of the present form if one works with the Borel-Serre compactification $\bar{X}$ : Since $\bar{X}$ is homotopy equivalent to $X$, one can still identify $\Gamma$ with the fundamental group of $\bar{X}$ and moreover the universal covering of $\bar{X}$ is contractible. We remark in bypassing that this is one of the main reasons why we study the Reidemeister torsion of $\bar{X}$ and not for example of a compactification of $X$ obtained by collapsing the boundary of $\bar{X}$ to a point. Now, although the (regularized) analytic torsion can in general not be computed explicitly for a fixed locally symmetric space and strongly acyclic coefficient systems, its asymptotic behaviour can be determined explicitly if either the manifold or the local system varies. In the compact case, results about the asymptotic behaviour of the analytic torsion were established by Bergeron and Venkatesh [BV], by Bismut, Ma and Zhang [BMZ] and by Müller and the author [MP1], MP3]. The extension of these results to the non-compact, finite volume real rank one case is due to Raimbault [Ra] and to Müller and the author [MP2], [MP5]. The result is always an asymptotic equality between analytic and $L^{2}$-torsion, where the latter is computed explicitly and is in particular not zero. Therefore, in the compact case, using the theorem of Cheeger [C1], Müller [Mü1], [Mü4] and Bismut and Zhang [BZ] on the equality 
of analytic and Reidemeister torsion, one can study the size of the torsion part in the cohomology of arithmetic groups by analytic methods, see [BV], [MM], [MP4]. Theorem 1.1 now implies that one can do this also in the non compact, finite-volume hyperbolic case.

Here we emphasize that in the non-compact case one also has to treat additionally the regulator, defined as in $[\mathrm{BV}]$, with respect to the Eisenstein cohomology classes. More precisely, the computations of section 8 show that one has to estimate the denominator of the C-matrix in the middle dimension which appears in Proposition 8.7. Using PoincaréLefschetz duality and the universal coefficient theorem, the problem of studying the image of the integral cohomology of the manifold in the integral cohomology of the boundary, which is related to denominators of Eisenstein series and seems to be much more difficult in general, can be avoided at least if one only wants to obtain exponential growth of torsion. Moreover, the regularized torsion of the cusps and the volume factors in Theorem 1.1 still need to be analyzed asymptotically.

We would now like to outline how the latter problem could be treated for example in the case where one is given a sequence $\Gamma_{i}, i \in \mathbb{N}$, of arithmetic groups which is exhaustive in a suitable sense; an example would be a tower, i.e. $\Gamma_{i+1} \subsetneq \Gamma_{i}$ for each $i$ and $\cap_{i} \Gamma_{i}=\{1\}$. We use the setup of the paper [MP5]. Let $X_{i}:=\Gamma_{i} \backslash \mathbb{H}^{d}$ be the underlying sequence of hyperbolic manifolds with cusps. Then one has to take into account that the normalization of the height functions in [MP5] is adapted to the covering, see [MP5, section 6]. We think that exactly this normalization and the arguments of [MP5, section 6] should allow to control the volumes $\operatorname{vol}\left(\partial F_{X_{i}}\right)$ in Theorem [1.1. For a treatment of the asymptotic behaviour of the regularized analytic torsion of the cusp we remark that the condition on cusp uniformity, which is due to Raimbault [Ra] and which is also posed in [MP5], ensures that the geometry of the boundary $\partial F_{X_{i}}$ does not degenerate as $i$ tends to infinity. Therefore, if one can express the torsion $\log T\left(F_{X_{i}}, \partial F_{X_{i}} ; E_{\rho}\right)$ in terms of special values of spectral zeta functions on the boundary $\partial F_{X_{i}}$ as indicated above, then we think that the same considerations as in [MP5, section 8], where the special values of Epstein zeta functions appearing on the geometric side of the trace formula were treated, guarantee that these values remain bounded in the covering. Since the number of cusps of $X_{i}$ grows of lower order than the volume of $X_{i}$ in the situations that we would like to consider [MP5], this would show that in the limit the logarithm of the torsion of the cusps divided by the volume goes to zero. We remark that at least in the case of a sequence of congruence subgroups of a Bianchi group $\mathrm{SL}_{2}\left(\mathcal{O}_{D}\right)$, the contribution $\log T\left(F_{X_{i}}, \partial F_{X_{i}} ; E_{\rho}\right)$ can even be treated in a simpler way. The point is that here, essentially as a consequence of the finiteness of the class number, there exists a finite set of lattices such that each lattice which defines a component of $\partial F_{X_{i}}$ arises by scaling one of these lattices, see [Ra, Proposition 4.3], [MP5, Proposition 11.1]. For this case, one can therefore also apply the gluing formula for the torsion on the cusps, where we refer to Corollary 15.4, equation (15.11) and Corollary 16.2.

This paper is organized as follows. In section 2 we fix some notations concerning the manifolds and representations we work with. In section 3 we introduce the bundles we work with. Sections 4, 5 and 6] are devoted to an analysis of the De Rham complex of the 
flat vector bundles restricted to the cusp, which is needed in order to define the Eisenstein cohomology classes and to compute the torsion of the long exact cohomology sequence. In section 7 we recall some properties of Eisenstein series used in the present context. In section 8 we give the explicit description of the cohomology in terms of Eisenstein series. In section 9 we introduce the Reidemeister torsion with respect to Eisenstein cohomology classes. In section 10 we give the definition of the relative and regularized traces and establish some of their analytic properties. In section 11 we establish results about the heat kernels which are important for our proof of the gluing formula. In section 12 we introduce the variation of the De Rham complex. In section 13 we analyze the construction of the heat kernel for the perturbed Laplacians. In section 14 we prove the gluing formula under the additional assumption that our metrics are of product structure in a neighbourhood of the set where the manifold is divided. In section 15 we drop this assumption on the metrics and the anomaly term will appear. In the final section 16 we compute the torsion of the long exact cohomology sequence that appears in the gluing formula explicitly.

Acknowledgement. I would like to thank Werner Müller for his constant interest in my work and for several useful discussions.

\section{Preliminaries}

2.1. Let $d=2 n+1$ be odd and let $G:=\operatorname{Spin}(d, 1), K:=\operatorname{Spin}(d)$ or $G:=\operatorname{SO}^{0}(d, 1)$, $K:=\mathrm{SO}(d)$, and $\widetilde{X}:=G / K$. Let $\mathfrak{g}$ and $\mathfrak{k}$ be the Lie algebras of $G$ and $K$ and let $\theta$ be the standard Cartan involution of $\mathfrak{g}$ associated to $K$ and let $\mathfrak{g}=\mathfrak{k} \oplus \mathfrak{p}$ be the Cartan decomposition of $\mathfrak{g}$. Let $B$ be the Killing form of $\mathfrak{g}$ and put

$$
<X, Y>_{\theta}:=-\frac{1}{2(d-1)} B(X, \theta(Y)), \quad X, Y \in \mathfrak{g} .
$$

Then the restricion of $<\cdot, \cdot>$ to $\mathfrak{p}$ induces a $G$-invariant metric on $\tilde{X}$. Equipped with this metric, $\widetilde{X}$ is isometric to the hyperbolic $d$-space $\mathbb{H}^{d}$ which carries the metric of constant curvature -1 .

2.2. Let $G=N A K$ be the Iwasawa decomposition of $G$ as in [MP2, section 2] and let $M$ be the centralizer of $A$ in $K$. Let $\mathfrak{g}, \mathfrak{n}, \mathfrak{a}, \mathfrak{k}, \mathfrak{m}$ denote the Lie algebras of $G, N, A K$ and M. Fix a Cartan subalgebra $\mathfrak{b}$ of $\mathfrak{m}$. Then $\mathfrak{h}:=\mathfrak{a} \oplus \mathfrak{b}$ is a Cartan subalgebra of $\mathfrak{g}$. We can identify $\mathfrak{g}_{\mathbb{C}} \cong \mathfrak{s o}(d+1, \mathbb{C})$. Let $e_{1} \in \mathfrak{a}^{*}$ be the positive restricted root defining $\mathfrak{n}$. Then we fix $e_{2}, \ldots, e_{n+1} \in i \mathfrak{b}^{*}$ such that the positive roots $\Delta^{+}\left(\mathfrak{g}_{\mathbb{C}}, \mathfrak{h}_{\mathbb{C}}\right)$ of the roots $\Delta\left(\mathfrak{g}_{\mathbb{C}}, \mathfrak{h}_{\mathbb{C}}\right)$ are chosen as in [Kn2, page 684-685] for the root system $D_{n+1}$. Let $W_{G}$ be the Weyl group of $\Delta\left(\mathfrak{g}_{\mathbb{C}}, \mathfrak{h}_{\mathbb{C}}\right)$. We let $\Delta^{+}\left(\mathfrak{g}_{\mathbb{C}}, \mathfrak{a}_{\mathbb{C}}\right)$ be the set of roots of $\Delta^{+}\left(\mathfrak{g}_{\mathbb{C}}, \mathfrak{h}_{\mathbb{C}}\right)$ which do not vanish on $\mathfrak{a}_{\mathbb{C}}$. The positive roots $\Delta^{+}\left(\mathfrak{m}_{\mathbb{C}}, \mathfrak{b}_{\mathbb{C}}\right)$ are chosen such that they are restrictions of elements from $\Delta^{+}\left(\mathfrak{g}_{\mathbb{C}}, \mathfrak{h}_{\mathbb{C}}\right)$

For $j=1, \ldots, n+1$ let

$$
\rho_{j}:=n+1-j
$$


Then the half-sums of positive roots $\rho_{G}$ and $\rho_{M}$, respectively, are given by

$$
\rho_{G}:=\frac{1}{2} \sum_{\alpha \in \Delta^{+}\left(\mathfrak{g}_{\mathbb{C}}, \mathfrak{h}_{\mathbb{C}}\right)} \alpha=\sum_{j=1}^{n+1} \rho_{j} e_{j} ; \quad \rho_{M}:=\frac{1}{2} \sum_{\alpha \in \Delta^{+}\left(\mathfrak{m}_{\mathbb{C}}, \mathfrak{b}_{\mathbb{C}}\right)} \alpha=\sum_{j=2}^{n+1} \rho_{j} e_{j} .
$$

2.3. Let $\mathbb{Z}\left[\frac{1}{2}\right]^{j}$ be the set of all $\left(k_{1}, \ldots, k_{j}\right) \in \mathbb{Q}^{j}$ such that either all $k_{i}$ are integers or all $k_{i}$ are half integers. Let $\operatorname{Rep}(G)$ denote the set of finite-dimensional irreducible representations $\rho$ of $G$. These are parametrized by their highest weights

$$
\Lambda(\rho)=k_{1}(\rho) e_{1}+\cdots+k_{n+1}(\rho) e_{n+1} ; \quad k_{1}(\rho) \geq k_{2}(\rho) \geq \cdots \geq k_{n}(\rho) \geq\left|k_{n+1}(\rho)\right|
$$

where $\left(k_{1}(\rho), \ldots, k_{n+1}(\rho)\right)$ belongs to $\mathbb{Z}\left[\frac{1}{2}\right]^{n+1}$ if $G=\operatorname{Spin}(d, 1)$ and to $\mathbb{Z}^{n+1}$ if $G=$ $\mathrm{SO}^{0}(d, 1)$. Moreover, the finite-dimensional irreducible representations $\nu \in \hat{K}$ of $K$ are parametrized by their highest weights

$$
\Lambda(\nu)=k_{2}(\nu) e_{2}+\cdots+k_{n+1}(\nu) e_{n+1} ; \quad k_{2}(\nu) \geq k_{3}(\nu) \geq \cdots \geq k_{n}(\nu) \geq k_{n+1}(\nu) \geq 0,
$$

where $\left(k_{2}(\nu), \ldots, k_{n+1}(\nu)\right)$ belongs to $\mathbb{Z}\left[\frac{1}{2}\right]^{n}$ if $G=\operatorname{Spin}(d, 1)$ and to $\mathbb{Z}^{n}$ if $G=\mathrm{SO}^{0}(d, 1)$. Finally, the finite-dimensional irreducible representations $\sigma \in \hat{M}$ of $M$ are parametrized by their highest weights

$$
\Lambda(\sigma)=k_{2}(\sigma) e_{2}+\cdots+k_{n+1}(\sigma) e_{n+1} ; \quad k_{2}(\sigma) \geq k_{3}(\sigma) \geq \cdots \geq k_{n}(\sigma) \geq\left|k_{n+1}(\sigma)\right|,
$$

where $\left(k_{2}(\sigma), \ldots, k_{n+1}(\sigma)\right)$ belongs to $\mathbb{Z}\left[\frac{1}{2}\right]^{n}$, if $G=\operatorname{Spin}(d, 1)$, and to $\mathbb{Z}^{n}$, if $G=\mathrm{SO}^{0}(d, 1)$. For $\nu \in \hat{K}$ and $\sigma \in \hat{M}$ we denote by $[\nu: \sigma]$ the multiplicity of $\sigma$ in the restriction of $\nu$ to $M$. For $\rho \in \operatorname{Rep} G$ let $\rho_{\theta}:=\rho \circ \theta$. Let $\Lambda(\rho)$ denote the highest weight of $\rho$ as in (2.4). Then the highest weight $\Lambda\left(\rho_{\theta}\right)$ of $\rho_{\theta}$ is given by

$$
\Lambda\left(\rho_{\theta}\right)=k_{1}(\rho) e_{1}+\cdots+k_{n}(\rho) e_{n}-k_{n+1}(\rho) e_{n+1} .
$$

2.4. Let $M^{\prime}$ be the normalizer of $A$ in $K$ and let $W(A)=M^{\prime} / M$ be the restricted Weylgroup. It has order two and it acts on the finite-dimensional representations of $M$ as follows. Let $w_{0} \in W(A)$ be the non-trivial element and let $m_{0} \in M^{\prime}$ be a representative of $w_{0}$. Given $\sigma \in \hat{M}$, the representation $w_{0} \sigma \in \hat{M}$ is defined by

$$
w_{0} \sigma(m)=\sigma\left(m_{0} m m_{0}^{-1}\right), \quad m \in M .
$$

If $d=2 n+2$ one has $w_{0} \sigma \cong \sigma$ for every $\sigma \in \hat{M}$. Assume that $d=2 n+1$. Let $\Lambda(\sigma)=k_{2}(\sigma) e_{2}+\cdots+k_{n+1}(\sigma) e_{n+1}$ be the highest weight of $\sigma$ as in (2.6). Then the highest weight $\Lambda\left(w_{0} \sigma\right)$ of $w_{0} \sigma$ is given by

$$
\Lambda\left(w_{0} \sigma\right)=k_{2}(\sigma) e_{2}+\cdots+k_{n}(\sigma) e_{n}-k_{n+1}(\sigma) e_{n+1} .
$$


2.5. Let $P_{0}:=N A M$. We equip $\mathfrak{a}$ with the norm induced from the restriction of the normalized Killing form on $\mathfrak{g}$. Let $H_{1} \in \mathfrak{a}$ be the unique vector which is of norm one and such that the positive restricted root, implicit in the choice of $N$, is positive on $H_{1}$. Let $\exp : \mathfrak{a} \rightarrow A$ be the exponential map. Every $a \in A$ can be written as $a=\exp \log a$, where $\log a \in \mathfrak{a}$ is unique. For $t \in \mathbb{R}$, we let $a(t):=\exp \left(t H_{1}\right)$. If $g \in G$, we define $n(g) \in N$, $H(g) \in \mathbb{R}$ and $\kappa(g) \in K$ by

$$
g=n(g) a(H(g)) \kappa(g) .
$$

Let $P$ be any parabolic subgroup. Then there exists a $k_{P} \in K$ such that $P=N_{P} A_{P} M_{P}$ with $N_{P}=k_{P} N k_{P}^{-1}, A_{P}=k_{P} A k_{P}^{-1}, M_{P}=k_{P} M k_{P}^{-1}$. We choose a set of $k_{P}$ 's, which will be fixed from now on. Let $k_{P_{0}}=1$. We let $a_{P}(t):=k_{P} a(t) k_{P}^{-1}$. If $g \in G$, we define $n_{P}(g) \in N_{P}, H_{P}(g) \in \mathbb{R}$ and $\kappa_{P}(g) \in K$ by

$$
g=n_{P}(g) a_{P}\left(H_{P}(g)\right) \kappa_{P}(g)
$$

and we define an identification $\iota_{P}$ of $(0, \infty)$ with $A_{P}$ by $\iota_{P}(t):=a_{P}(\log (t))$. For $Y>0$, let $A_{P}^{0}[Y]:=\iota_{P}(Y, \infty)$ and $A_{P}[Y]:=\iota_{P}[Y, \infty)$. For $g \in G$ as in (2.9) we let $y_{P}(g):=e^{H_{P}(g)}$.

2.6. We parametrize the principal series as follows. Given $\sigma \in \hat{M}$ with $\left(\sigma, V_{\sigma}\right) \in \sigma$, let $\mathcal{H}^{\sigma}$ denote the space of measurable functions $f: K \rightarrow V_{\sigma}$ satisfying

$$
f(m k)=\sigma(m) f(k), \quad \forall k \in K, \forall m \in M, \quad \text { and } \quad \int_{K}\|f(k)\|^{2} d k=\|f\|^{2}<\infty .
$$

Then for $\lambda \in \mathbb{C}$ and $f \in H^{\sigma}$ let

$$
\pi_{\sigma, \lambda}(g) f(k):=e^{(i \lambda+(d-1) / 2) H(k g)} f(\kappa(k g)) .
$$

Then the representations $\pi_{\sigma, \lambda}$ are unitary iff $\lambda \in \mathbb{R}$. Moreover, for $\lambda \in \mathbb{R}-\{0\}$ and $\sigma \in \hat{M}$ the representations $\pi_{\sigma, \lambda}$ are irreducible and $\pi_{\sigma, \lambda}$ and $\pi_{\sigma^{\prime}, \lambda^{\prime}}, \lambda, \lambda^{\prime} \in \mathbb{C}$ are equivalent iff either $\sigma=\sigma^{\prime}, \lambda=\lambda^{\prime}$ or $\sigma^{\prime}=w_{0} \sigma, \lambda^{\prime}=-\lambda$. We use this parametrization of the principal series in order to stay consistent with the notation of [MP1], [MP2], [MP5]. For $\sigma \in \hat{M}$ with highest weight $\Lambda(\sigma)$ given by (2.6), let

$$
c(\sigma):=\sum_{j=2}^{n+1}\left(k_{j}(\sigma)+\rho_{j}\right)^{2}-\sum_{j=1}^{n+1} \rho_{j}^{2} .
$$

Then for the Casimir element $\Omega \in Z\left(\mathfrak{g}_{\mathbb{C}}\right)$ one has

$$
\pi_{\sigma, \lambda}(\Omega)=-\lambda^{2}+c(\sigma),
$$

[MP1, Corollary 2.4]. If $V$ is a $(\mathfrak{g}, K)$-module, defined as in [BW][section 0.2], we let $H^{*}(\mathfrak{g}, K: V)$ denote its $(\mathfrak{g}, K)$-cohomology groups. If one lets

$$
C^{q}(\mathfrak{g}, K ; V):=\operatorname{Hom}_{K}\left(\Lambda^{q}(\mathfrak{g} / \mathfrak{k}), V_{\rho}\right),
$$

then the $C^{q}(\mathfrak{g}, K ; V)$ naturally form a cochain complex $C^{*}(\mathfrak{g}, K ; V)$ whose cohomology groups can be identified with the groups $H^{*}(\mathfrak{g}, K ; V)$, [BW] [Chapter I, section 1]. By $\mathcal{H}_{K}^{\sigma, \lambda}$ we shall denote the $(\mathfrak{g}, K)$-module associated to $\pi_{\sigma, \lambda}$ which is formed by the $K$-infinite vectors in $\mathcal{H}^{\sigma}$. 
2.7. Now let $X$ be an oriented hyperbolic manifold. Then there exists a discrete, torsionfree subgroup $\Gamma$ of $G$ such that

$$
X=\Gamma \backslash \tilde{X}
$$

One can canonically identify $\Gamma$ with the fundamental group of $X$. We assume that $X$ is of finite volume but we do not assume that $X$ is compact. A parabolic subgroup $P$ of $G$ is called $\Gamma$-cuspidal if $\Gamma \cap N_{P}$ is a lattice in $N_{P}$. Let $\mathfrak{P}_{\Gamma}$ be a fixed set of representatives of the $\Gamma$-conjugacy classes of $\Gamma$-cuspidal parabolic subgroups of $G$. Then $\mathfrak{P}_{\Gamma}$ is finite and $\kappa(\Gamma):=\# \mathfrak{P}_{\Gamma}$ equals the number of cusps of $X$; the manifold $X$ is non-compact if and only if $\kappa(\Gamma)>0$. For convenience we assume that for each $\Gamma$-cuspidal parabolic subgroup $P=N_{P} A_{P} M_{P}$ of $G$ one has

$$
\Gamma \cap P=\Gamma \cap N_{P}
$$

This condition is satisfied for example if $\Gamma$ is "neat", which means that the group generated by the eigenvalues of any $\gamma \in \Gamma$ contains no roots of unity $\neq 1$. It thus holds for many groups $\Gamma$ which are of arithmetic significance: For example, if $F$ is an imaginary quadratic number field with ring of integers $\mathcal{O}_{F}$ and $\mathfrak{a}$ is a non-zero ideal in $\mathcal{O}_{F}$, then the associated principal congruence subgroup $\Gamma(\mathfrak{a})$ of $\mathrm{SL}_{2}\left(\mathcal{O}_{F}\right)$ satisfies assumption (2.11) for all ideals $\mathfrak{a}$ whose norm is greater than a given constant that depends only on $F$. Similarly, in higher dimensions, if $G:=\mathrm{SO}^{0}(d, 1)$ assumption (2.11) holds for all principal congruence subgroups $\Gamma(q)$ of $G(\mathbb{Z})$ associated to $q \in \mathbb{N}$ with $q \geq 3$, see [Bo1, 17.4].

Assumption (2.11) was made in the paper [MP2], where the regularized analytic torsion was defined for the present context. However, in that paper it was mainly put in order to study the asymptotic behaviour of the analytic torsion along rays in the weight lattice, which relied on Fourier inversion formulas on the geometric side of the trace formula. For the pure short-time asymptotic expansion of the heat traces, it is certainly not necessary. We think that it can be dropped without an essential change of the methods used in the present paper.

The geometry of the quotient $\Gamma \backslash G$ and of the manifold $X$ can be described as follows. Firstly, there exists a $Y_{0}>0$ and for every $Y \geq Y_{0}$ a compact connected subset $C(Y)$ of $G$ such that in the sense of a disjoint union one has

$$
G=\Gamma \cdot C(Y) \sqcup \bigsqcup_{P_{i} \in \mathfrak{P}_{\Gamma}} \Gamma \cdot N_{P_{i}} A_{P_{i}}^{0}[Y] K
$$

and such that for each $P_{i} \in \mathfrak{P}_{\Gamma}$ one has

$$
\gamma \cdot N_{P_{i}} A_{P_{i}}^{0}[Y] K \cap N_{P_{i}} A_{P_{i}}^{0}[Y] K \neq \emptyset \Leftrightarrow \gamma \in \Gamma \cap N_{P_{i}}
$$

For $Y \in(0, \infty)$ and $P_{i} \in \mathfrak{P}_{\Gamma}$ we let

$$
F_{P}\left(Y_{i}\right):=A_{P_{i}}[Y] \times \Gamma \cap N_{P_{i}} \backslash N_{P_{i}} \cong[Y, \infty) \times \Gamma \cap N_{P_{i}} \backslash N_{P_{i}} .
$$


It follows from (2.12) and (2.13) that for each $Y \geq Y_{0}$ there exists a compact manifold $X(Y)$ with smooth boundary such that $X$ has a decomposition as

$$
X=X(Y) \cup \bigsqcup_{P_{i} \in \mathfrak{P}_{\Gamma}} F_{P_{i}}(Y)
$$

with $X(Y) \cap F_{P_{i}, Y}=\partial X(Y) \cap \partial F_{P_{i}}(Y)=\partial F_{P_{i}}(Y)$ and $F_{P_{i}}(Y) \cap F_{P_{j}}(Y)=\emptyset$ if $i \neq j$ and with $\partial X(Y)=\sqcup_{P_{i} \in \mathfrak{P}_{\Gamma}} \partial F_{P_{i}}(Y)$. Let $g_{N_{P_{i}}}$ be the pushdown of the invariant metric on $N_{P_{i}}$ induced by the metric in (2.1) to $\Gamma \cap N_{P_{i}} \backslash N_{P_{i}}$. Then the metric on $F_{P_{i}}(Y)$ is given by

$$
\frac{1}{y^{2}} d y^{2}+\frac{1}{y^{2}} g_{N_{P_{i}}}
$$

Since $N_{P_{i}}$ is abelian in the present case, $\Gamma \cap N_{P_{i}} \backslash N_{P_{i}}$ with the metric $g_{N_{P_{i}}}$ is isometric to a flat torus. Equations (2.15) and (2.16) describe the geometry of $X$ as a manifold with cusps. We define the Borel-Serre compactification $\bar{X}$ of $X$ as the diffeomorphism class of the manifolds $X(Y), Y \geq Y_{0}$. Thus $\bar{X}$ is a compact smooth manifold with boundary which is homotopy equivalent to $X$. For $Y \in(0, \infty)$ we shall use the notation

$$
F_{X}(Y):=\bigsqcup_{P_{i} \in \mathfrak{P}_{\Gamma}} F_{P_{i}}(Y)
$$

We furthermore let $F_{X}:=F_{X}(1)$. By (2.16), under the isomorphism $F_{P_{i}, Y} \cong[Y, \infty) \times \Gamma \cap$ $N_{P_{i}} \backslash N_{P_{i}}$, for $Y \geq Y_{0}$ one has

$$
\int_{X} f(x) d x=\int_{X(Y)} f(x) d x+\sum_{P_{i} \in \mathfrak{P}_{\Gamma}} \int_{Y}^{\infty} \int_{\Gamma \cap N_{P_{i}} \backslash N_{P_{i}}} y^{-(2 n+1)} f(y, v) d g_{N_{P_{i}}}(v) d y
$$

\section{Homogeneous And flat Vector Bundles}

Let $X$ be the manifold from the previous section. In this section we introduce the class of vector bundles over $X$ and $\tilde{X}$ we will be concerned with. These bundles were firstly introduced by Matsushima and Murakmami in [MaMu and we refer to this paper for further details.

Let $\nu$ be a finite-dimensional unitary representation of $K$ on $\left(V_{\nu},\langle\cdot, \cdot\rangle_{\nu}\right)$. Let $\tilde{E}_{\nu}:=$ $G \times{ }_{\nu} V_{\nu}$ be the associated homogeneous vector bundle over $\tilde{X}$. Then $\langle\cdot, \cdot\rangle_{\nu}$ induces a $G$ invariant metric $\tilde{h}_{\nu}$ on $\tilde{E}_{\nu}$. Let $E_{\nu}:=\Gamma \backslash\left(G \times{ }_{\nu} V_{\nu}\right)$ be the associated locally homogeneous bundle over $X$. Since $\tilde{h}_{\nu}$ is $G$-invariant, it can be pushed down to a fiber metric $h_{\nu}$ on $E_{\nu}$. Let

$$
C^{\infty}(G, \nu):=\left\{f: G \rightarrow V_{\nu}: f \in C^{\infty}, f(g k)=\nu\left(k^{-1}\right) f(g), \forall g \in G, \forall k \in K\right\}
$$

Let

$$
C^{\infty}(\Gamma \backslash G, \nu):=\left\{f \in C^{\infty}(G, \nu): f(\gamma g)=f(g), \forall g \in G, \forall \gamma \in \Gamma\right\} .
$$

Then the smooth sections $C^{\infty}\left(\tilde{X}, E_{\nu}\right)$ of $\tilde{E}_{\nu}$ are canonically isomorphic to $C^{\infty}(G, \nu)$. Similarly, for the sapce $C^{\infty}\left(X, E_{\nu}\right)$ of smooth sections of $E_{\nu}$ there is a canonical isomorphism 
$C^{\infty}\left(X, E_{\nu}\right) \cong C^{\infty}(\Gamma \backslash G, \nu)$ (see [Mi1, p. 4]). There is also a corresponding isometry for the space $L^{2}\left(X, E_{\nu}\right)$ of $L^{2}$-sections of $E_{\nu}$.

Let $\rho$ be an irreducible finite-dimensional representation of $G$ on $V_{\rho}$ and let $E_{\rho}:=$ $\widetilde{X} \times_{\left.\rho\right|_{\Gamma}} V_{\rho}$ be the flat vector bundle over $X$ associated to the restriction of $\rho$ to $\Gamma$. Let $\widetilde{E}_{\rho} \rightarrow \widetilde{X}$ be the homogeneous vector bundle over $\tilde{X}$ associated to $\left.\rho\right|_{K}$. Then by [MaMu, Proposition 3.1] there is a canonical isomorphism

$$
E_{\rho} \cong \Gamma \backslash \widetilde{E}_{\rho} .
$$

By [MaMu, Lemma 3.1], there exists an inner product $\langle\cdot, \cdot\rangle$ on $V_{\rho}$ such that

(1) $\langle\rho(Y) u, v\rangle=-\langle u, \rho(Y) v\rangle$ for all $Y \in \mathfrak{k}, u, v \in V_{\rho}$

(2) $\langle\rho(Y) u, v\rangle=\langle u, \rho(Y) v\rangle$ for all $Y \in \mathfrak{p}, u, v \in V_{\rho}$.

Such an inner product is called admissible. It is unique up to scaling. Fix an admissible inner product. Since $\left.\rho\right|_{K}$ is unitary with respect to this inner product, it induces a fibre metric on $\widetilde{E}_{\rho}$, and hence a fibre metric $h$ on $E_{\rho}$. This fibre metric will also be called admissible. Let $\Lambda^{p}\left(X, E_{\rho}\right)$ be the space of $E_{\rho}$-valued $p$-forms. This is the space of smooth sections of the vector bundle $\Lambda^{p}\left(E_{\rho}\right):=\Lambda^{p} T^{*} X \otimes E_{\rho}$. Let

$$
d_{p}(\rho): \Lambda^{p}\left(X, E_{\rho}\right) \rightarrow \Lambda^{p+1}\left(X, E_{\rho}\right)
$$

be the exterior derivative and let

$$
\Delta_{p}(\rho)=d_{p}(\rho)^{*} d_{p}(\rho)+d_{p-1}(\rho) d_{p-1}(\rho)^{*}
$$

be the Laplace operator on $E_{\rho}$-valued $p$-forms. Let $\nu_{p}(\rho)$ be the representation of $K$ defined by

$$
\nu_{p}(\rho):=\Lambda^{p} \operatorname{Ad}^{*} \otimes \rho: K \rightarrow \operatorname{GL}\left(\Lambda^{p} \mathfrak{p}^{*} \otimes V_{\rho}\right) .
$$

Then there is a canonical isomorphism $\Lambda^{p}\left(E_{\rho}\right) \cong \Gamma \backslash\left(G \times_{\nu_{p}(\rho)}\left(\Lambda^{p} \mathfrak{p}^{*} \otimes V_{\rho}\right)\right)$, which induces an isomorphism

$$
\Lambda^{p}\left(X, E_{\rho}\right) \cong C^{\infty}\left(\Gamma \backslash G, \nu_{p}(\rho)\right) .
$$

There is a corresponding isometry of the $L^{2}$-spaces. We let

$$
d: C^{\infty}\left(\Gamma \backslash G, \nu_{p}(\rho)\right) \rightarrow C^{\infty}\left(\Gamma \backslash G, \nu_{p+1}(\rho)\right)
$$

be the map which is induced by the map $d_{p}(\rho)$ under the isomorphism in (3.7) and we denote the corresponding complex by $\Omega^{*}(\Gamma \backslash G, \rho)$. Then there is a canonical isomorphism of complexes

$$
\Omega^{*}(\Gamma \backslash G, \rho) \cong C^{*}\left(\mathfrak{g}, K ; C^{\infty}(\Gamma \backslash G) \otimes V_{\rho}\right),
$$

where the complex on the right hand side is as in section 2.6, Let $\rho(\Omega)$ be the Casimir eigenvalue of $\rho$. Then with respect to the isomorphism (3.7) one has

$$
\Delta_{p}(\rho)=-\Omega+\rho(\Omega) \text { Id }
$$

(see [MaMu, (6.9)]). 


\section{The De Rham Complex on the CUSP}

Let $\Gamma$ be as in section 2.7 and let $P$ be a fixed $\Gamma$-cuspidal parabolic subgroup of $G$. In this section, we describe the De Rham complex with coefficients the flat vector bundles corresponding to the bundles $\tilde{E}_{\rho}$ over the cusp $F_{P}:=\left(\Gamma \cap N_{P}\right) \backslash G / K$ of infinite volume. Here we equip $F_{P}$ with the metric induced from the metric on $G / K$.

Without loss of generality, we assume that $P=P_{0}$ is the parabolic subgroup of $G$ from section 2.5 and to ease notation we shall write $\Gamma_{N}:=\Gamma \cap N$. Analogous to the notation of the previous section, for $\rho \in \operatorname{Rep}(G)$ we shall denote by $E_{\rho}:=\widetilde{X} \times_{\left.\rho\right|_{\Gamma_{N}}} V_{\rho}$ the flat vector bundle over $F_{P}$ induced by the restriction of $\rho$ to $\Gamma_{N}$. The restriction of $\rho$ to $K$ defines a locally homogeneous vector bundle $W_{\rho}:=\Gamma_{N} \backslash G \times_{\rho \mid K} V_{\rho}$ over $F_{P}$ and analogously to the situation of the previous section there is a canonical isomorphism $A: W_{\rho} \rightarrow E_{\rho}$. More precisely, $A$ is defined by putting

$$
A\left[\Gamma_{N} g, v\right]:=[g K, \rho(g) v],
$$

for an equivalence class $\left[\Gamma_{N} g, v\right] \in W_{\rho}, g \in G, v \in V_{\rho}$. The smooth sections of $W_{\rho}$ are canonically isomorphic to the space

$$
C^{\infty}\left(\Gamma_{N} \backslash G, \rho\right)=\left\{f: G \rightarrow V_{\rho}: f \in C^{\infty}: f(\gamma g k)=\rho\left(k^{-1}\right) f(g), \forall g \in G, \gamma \in \Gamma_{N}, k \in K\right\} .
$$

The smooth sections of $E_{\rho}$ are canonically isomorphic to the space

$$
C^{\infty}\left(G / K,\left.\rho\right|_{\Gamma_{N}}\right):=\left\{f: G \rightarrow V_{\rho}: f \in C^{\infty}: f(\gamma g k)=\rho(\gamma) f(g), \forall g \in G, k \in K, \gamma \in \Gamma_{N}\right\} .
$$

If we denote by $A: C^{\infty}\left(\Gamma_{N} \backslash G, \rho\right) \rightarrow C^{\infty}\left(G / K,\left.\rho\right|_{\Gamma_{N}}\right)$ the isomorphism corresponding to the isomorphism $A$ from (4.1), then for $f \in C^{\infty}\left(\Gamma_{N} \backslash G,\left.\rho\right|_{K}\right), h \in C^{\infty}\left(G / K,\left.\rho\right|_{\Gamma_{N}}\right)$ we have

$$
A f(g K)=\rho(g) f(g) ; \quad A^{-1} h\left(\Gamma_{N} g\right)=\rho\left(g^{-1}\right) h(g) .
$$

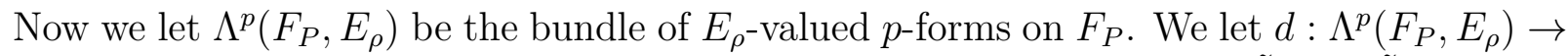
$\Lambda^{p+1}\left(F_{P}, E_{\rho}\right)$ be the exterior derivative. Then for smooth vector fields $\tilde{Y}_{1}, \ldots, \tilde{Y}_{p+1}$ on $F_{P}$ and $f \in \Lambda^{p}\left(F_{P}, E_{\rho}\right)$ we have

$$
\begin{aligned}
d f\left(\tilde{Y}_{1}, \ldots, \tilde{Y}_{p+1}\right) & =\sum_{i}(-1)^{i+1} \tilde{Y}_{i}\left(f\left(\tilde{Y}_{1}, \ldots, \hat{\tilde{Y}}_{i}, \ldots, \tilde{Y}_{p+1}\right)\right) \\
& +\sum_{i<j}(-1)^{i+j} f\left(\left[\tilde{Y}_{i}, \tilde{Y}_{j}\right], \tilde{Y}_{1}, \ldots, \hat{\tilde{Y}}_{i}, \ldots, \hat{\tilde{Y}}_{j}, \ldots, \tilde{Y}_{p+1}\right) .
\end{aligned}
$$

One has $\mathfrak{g} / \mathfrak{k} \cong \mathfrak{a} \oplus \mathfrak{n}$ and one denotes by Ad the action of $K$ on the right hand side which is induced by the corresponding action on the left hand side. Let

$$
\nu_{p}(\rho): K \rightarrow \operatorname{Aut}\left(\Lambda^{p}(\mathfrak{a} \oplus \mathfrak{n})^{*} \otimes V_{\rho}\right), \quad \nu_{p}(\rho)(k):=\Lambda^{p} \operatorname{Ad}^{*}(k) \otimes \rho(k) .
$$

Let $W_{\nu_{p}(\rho)}:=\Gamma_{N} \backslash G \times_{\nu_{p}(\rho)} \Lambda^{p}(\mathfrak{a} \oplus \mathfrak{n})^{*} \otimes V_{\rho}$ be the locally homogeneous bundle over $F_{P}$ induced by $\nu_{p}(\rho)$. Then $W_{\nu_{p}(\rho)}$ is canonically isomorphic to the bunde of $W_{\rho}$-valued $p$ forms on $F_{P}$. Moreover, the smooth section of $W_{\nu_{p}(\rho)}$ are canonically isomorphic to the 
space $C^{\infty}\left(\Gamma_{N} \backslash G, \nu_{p}(\rho)\right)$ which is defined as the space $C^{\infty}\left(\Gamma_{N} \backslash G,\left.\rho\right|_{K}\right)$, replacing $\left.\rho\right|_{K}$ by $\nu_{p}(\rho)$. We let

$$
d: C^{\infty}\left(\Gamma_{N} \backslash G, \nu_{p}(\rho)\right) \rightarrow C^{\infty}\left(\Gamma_{N} \backslash G, \nu_{p+1}(\rho)\right)
$$

be the map which is induced by the exterior derivative under the canonical identification of these space with $\Lambda^{p}\left(F_{P}, E_{\rho}\right)$ resp. $\Lambda^{p+1}\left(F_{P}, E_{\rho}\right)$ and we denote the corresponding complex by $\Omega^{*}\left(\Gamma_{N} \backslash G, \rho\right)$. Then it follows easily from (4.2) and (4.3) that for any vectors $T_{1}, \ldots, T_{p+1} \in(\mathfrak{a} \oplus \mathfrak{n})$ and all $\phi \in C^{\infty}\left(\Gamma_{N} \backslash G, \nu_{p}(\rho)\right)$ we have

$$
\begin{aligned}
d \phi\left(T_{1}, \ldots, T_{p+1}\right) & =\sum_{i}(-1)^{i+1}\left(\tilde{T}_{i}+\rho\left(T_{i}\right)\right)\left(\phi\left(T_{1}, \ldots, \hat{T}_{i}, \ldots, T_{p+1}\right)\right) \\
& +\sum_{i<j}(-1)^{i+j} \phi\left(\left[T_{i}, T_{j}\right], T_{1}, \ldots, \hat{T}_{i}, \ldots, \hat{T}_{j}, \ldots, T_{p+1}\right),
\end{aligned}
$$

where $\tilde{T}_{i}$ denotes the left-invariant vector field on $G$ induced by $T_{i}$.

Remark 4.1. If $\mathfrak{g}=\mathfrak{k} \oplus \mathfrak{p}$ is the Cartan decompostion of $\mathfrak{g}$ with respect to $\theta$ and if one works with vector-fields constructed out of elements of $\mathfrak{p}$, as it is done in $\mathrm{MaMu}$, Proposition 4.1], then the last line in (4.4) is not present since one always has $[\mathfrak{p}, \mathfrak{p}] \subset \mathfrak{k}$.

Finally, there is a canonical isomorphism of complexes

$$
\Omega^{*}\left(\Gamma_{N} \backslash G, \rho\right) \cong C^{*}\left(\mathfrak{g}, K ; C^{\infty}\left(\Gamma_{N} \backslash G\right) \otimes V_{\rho}\right),
$$

where the complex on the right hand side is as in section (2.6). Here the action of $G$ on $C^{\infty}\left(\Gamma_{N} \backslash G\right) \otimes V_{\rho}$ is the tensor product of the right-regular representation of $G$ and the representation $\rho$. The isomorphism in (4.5) is defined as follows. To $f \in \Omega^{*}\left(\Gamma_{N} \backslash G, \nu_{p}(\rho)\right)$ one associates the element in $\operatorname{Hom}_{K}\left(\Lambda^{p}(\mathfrak{g} / \mathfrak{k}) ; C^{\infty}\left(\Gamma_{N} \backslash G\right) \otimes V_{\rho}\right)$ which assigns to $X \in \Lambda^{p}(\mathfrak{g} / \mathfrak{k})$ the $V_{\rho^{-v a l u e d}}$ function $f_{X}$ on $G$ given by $f_{X}(g):=f(g)(X)$. Here one uses the canonical isomorphisms of $K$-modules: $\Lambda^{p}(\mathfrak{a} \oplus \mathfrak{n})^{*} \otimes V_{\rho} \cong \operatorname{Hom}\left(\Lambda^{p}(\mathfrak{a} \oplus \mathfrak{n}), V_{\rho}\right) \cong \operatorname{Hom}\left(\Lambda^{p}(\mathfrak{g} / \mathfrak{k}), V_{\rho}\right)$.

\section{LIFTS OF DIFFERENTIAL FORMS FROM THE BOUNDARY TO THE CUSP}

We keep the notations of the previous section. We let $E_{\rho}^{0}:=N \times_{\left.\rho\right|_{\Gamma_{N}}} V_{\rho}$ be the flat vector bundle over $\Gamma_{N} \backslash N$ induced by $\left.\rho\right|_{\Gamma_{N}}$. We can identify the smooth sections of $E_{\rho}^{0}$ with the space

$$
C^{\infty}\left(N,\left.\rho\right|_{\Gamma_{N}}\right):=\left\{f: N \rightarrow V_{\rho}: f \in C^{\infty}: f(\gamma n)=\rho(\gamma) f(n), \forall \gamma \in \Gamma_{N}, \forall n \in N\right\} .
$$

We let $Y_{1}, \ldots, Y_{2 n}$ be a basis of $\mathfrak{n}$ and we let $\tilde{Y}_{1}, \ldots, \tilde{Y}_{2 n}$ denote the corresponding invariant

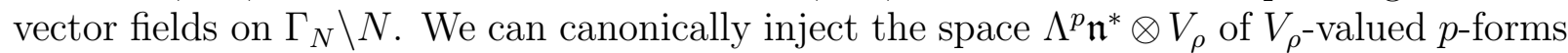
on $\mathfrak{n}$ into the space of $E_{\rho}^{0}$-valued $p$-forms on $\Gamma_{N} \backslash N$. Namely, to any $\Phi \in \Lambda^{p} \mathfrak{n}^{*} \otimes V_{\rho}$ we associate the unique $E_{\rho}^{0}$-valued $p$-form $\Phi$ on $\Gamma_{N} \backslash N$ such that one has in $C^{\infty}\left(N,\left.\rho\right|_{\Gamma_{N}}\right)$ :

$$
\Phi\left(\Gamma_{N} n\right)\left(\tilde{Y}_{i_{1}}, \ldots, \tilde{Y}_{i_{p}}\right):=\rho(n) \Phi\left(Y_{i_{1}}, \ldots, Y_{i_{p}}\right), \quad \forall n \in N
$$

Here $\Gamma_{N} n$ denotes the image of $n$ in $\Gamma_{N} \backslash N$. This form exists since in our case $N$ is abelian otherwise one would have to additionally twist with the adjoint action of $N$ on $\mathfrak{n}$. The maps in (5.1) also induce a chain map between the $V_{\rho}$-valued Lie-algebra cohomology of $\mathfrak{n}$ and 
the De-Rham complex of $E_{\rho}^{0}$-valued differential forms on $\Gamma_{N} \backslash N$. By Van Est's Theorem $\mathrm{vE}$, the corresponding map on the cohomology is an isomorphism.

Next we lift $V_{\rho}$-valued $p$-foms on $\mathfrak{n}$ to differential forms on $F_{P}$. The space $\Lambda^{p}(\mathfrak{n})^{*}$ is not invariant under $\operatorname{Ad}(K)$. However, we can regard the space $\Lambda^{p}(\mathfrak{n})^{*} \otimes V_{\rho}$ as a subspace of $\Lambda^{p}(\mathfrak{n} \oplus \mathfrak{a})^{*} \otimes V_{\rho}$ and thus to $\Phi \in \Lambda^{p}(\mathfrak{n})^{*} \otimes V_{\rho}$ we can associate the element

$$
\Phi_{\lambda} \in C^{\infty}\left(\Gamma_{N} \backslash G, \nu_{p}(\rho)\right), \quad \Phi_{\lambda}(n a(t) k):=e^{(\lambda+(d-1) / 2) t} \nu_{p}(\rho)\left(k^{-1}\right) \Phi,
$$

where $n \in N, a(t) \in A$ as in section 2.5, $k \in K$. Let $H_{1} \in \mathfrak{a}$ be as in section 2.5. Then the differential of $\Phi_{\lambda}$ is given as follows.

Lemma 5.1. For $n \in N, a(t) \in A$ and $k \in K$ we have

$$
d \Phi_{\lambda}(n a(t) k)=e^{\left(\lambda+\frac{d-1}{2}\right) t} \nu_{p+1}(\rho)\left(k^{-1}\right)\left(d H_{1} \wedge\left((\lambda+(d-1) / 2) \Phi+\left(\Lambda^{p} \operatorname{Ad}^{*} \otimes \rho\right)\left(H_{1}\right) \Phi\right)\right) .
$$

Proof. To prove the Lemma, we can assume that $k=1$. By the definition of $\Phi_{\lambda}$, it suffices to evaluate $d \Phi_{\lambda}$ on $p+1$ - tuples $\left(H_{1}, Y_{1}, \ldots, Y_{p}\right)$, where $Y_{1}, \ldots, Y_{p} \in \mathfrak{n}$. Applying (4.4) and using that the interior multiplication of $\Phi_{\lambda}$ with $H_{1}$ vanishes, we obtain

$$
\begin{aligned}
d \Phi_{\lambda}(n a(t))\left(H_{1}, Y_{1}, \ldots, Y_{p}\right)= & e^{\left(\lambda+\frac{d-1}{2}\right) t}\left((\lambda+(d-1) / 2) \Phi\left(Y_{1}, \ldots, Y_{p}\right)+\rho\left(H_{1}\right) \Phi\left(Y_{1}, \ldots, Y_{p}\right)\right) \\
& +\sum_{i=1}^{p}(-1)^{i} \Phi\left(\left[H_{1}, Y_{i}\right], \ldots, \hat{Y}_{i}, \ldots, Y_{p}\right) .
\end{aligned}
$$

For the representation $\Lambda^{p} \operatorname{Ad}^{*} \otimes \rho: \mathfrak{a} \rightarrow \operatorname{End}\left(\Lambda^{p}(\mathfrak{n})^{*} \otimes V_{\rho}\right)$ one has

$$
\left(\Lambda^{p} \mathrm{Ad}^{*} \otimes \rho\right)\left(H_{1}\right) \Phi\left(Y_{1}, \ldots, Y_{p}\right)=-\sum_{i} \Phi\left(Y_{1}, \ldots,\left[H_{1}, Y_{i}\right], \ldots, Y_{p}\right)+\rho\left(H_{1}\right) \Phi\left(Y_{1}, \ldots, Y_{p}\right)
$$

and the Lemma follows.

For every $a \in A$ we have an inclusion $\iota_{a}: \Gamma_{N} \backslash N \hookrightarrow F_{P}$ given by $\iota_{a}\left(\Gamma_{N} n\right):=\Gamma_{N} n a K$, where $\Gamma_{N} n$ resp. $\Gamma_{N} n a K$ denote the equivalence classes of $n$ in $\Gamma_{N} \backslash N$ resp. $n a$ in $\Gamma_{N} \backslash G / K$. Let $\iota_{a}^{*} E_{\rho}$ be the corresponding pullback bundle of $E_{\rho}$. Then $\iota_{a}^{*} E_{\rho}$ is isomorphic to $H_{\rho}^{0}$. Thus also $\iota_{a}^{*} W_{\rho}$ is isomorphic to $H_{\rho}^{0}$, where $W_{\rho}$ is the locally homogeneous bundle from the previous section. Let $\iota_{a}^{*}: C^{\infty}\left(\Gamma_{N} \backslash G,\left.\rho\right|_{K}\right) \rightarrow C^{\infty}\left(N ;\left.\rho\right|_{\Gamma_{N}}\right)$ be the map between the smooth sections induced by $\iota_{a}$. Then, by (4.2), for $f \in C^{\infty}\left(\Gamma_{N} \backslash G,\left.\rho\right|_{K}\right)$ one has

$$
\iota_{a}^{*} f(n)=\rho(n a) f(n a) .
$$

The pullback of $\Phi_{\lambda}$ under $\iota_{a}$ is computed as follows.

Lemma 5.2. For every $a(t) \in A$ we have

$$
\iota_{a(t)}^{*} \Phi_{\lambda}=e^{(\lambda+(d-1) / 2) t}\left(\Lambda^{p} \operatorname{Ad}^{*} \otimes \rho\right)(a(t)) \Phi .
$$

Proof. We identify elements of the Lie algebra $\mathfrak{n}$ with the corresponding left invariant vector fields on $N$ resp. $G$. Let $\pi_{1}: N \rightarrow \Gamma_{N} \backslash N, \pi_{2}: G \rightarrow \Gamma_{N} \backslash G / K$ be the projections. Then for $Y \in \mathfrak{n}$ one has $\left(\iota_{a}\right)_{*}\left(\pi_{1, *}(Y)\right)=\pi_{2, *}\left(\operatorname{Ad}\left(a^{-1}\right) Y\right)$. Thus the Lemma follows immediately from equation (5.1) and equation (5.3). 


\section{Closed forms on the CUSP}

We consider again the representation $\Lambda^{p} \operatorname{Ad}^{*} \otimes \rho$ of $M A$ on $\Lambda^{p} \mathfrak{n}^{*} \otimes V_{\rho}$. For $\sigma \in \hat{M}$, we denote by $\left(\Lambda^{p} \mathfrak{n}^{*} \otimes V_{\rho}\right)_{\sigma}$ the $\sigma$-isotypical component of the representation $\Lambda^{p} \operatorname{Ad}^{*} \otimes \rho$ restricted to $M$; we have thus a decomposition

$$
\Lambda^{p} \mathfrak{n}^{*} \otimes V_{\rho}=\bigoplus_{\sigma \in \hat{M}}\left(\Lambda^{p} \mathfrak{n}^{*} \otimes V_{\rho}\right)_{\sigma}
$$

of $\Lambda^{p} \mathfrak{n}^{*} \otimes V_{\rho}$, where only finitely many spaces $\left(\Lambda^{p} \mathfrak{n}^{*} \otimes V_{\rho}\right)_{\sigma}$ are not zero. Using the preceding computations and Kostant's theorem, one can now construct closed and harmonic forms on $F_{P}$ with values in $E_{\rho}$. Let $\rho \in \hat{G}$ and let $\Lambda(\rho)=\rho_{1} e_{1}+\cdots+\rho_{n+1} e_{n+1}$ be its highest weight as in (2.4). For $w \in W_{G}$ let $l(w)$ denote its length with respect to the simple roots which define the positive roots $\Delta^{+}\left(\mathfrak{g}_{\mathbb{C}}, \mathfrak{h}_{\mathbb{C}}\right)$. Let

$$
W^{1}:=\left\{w \in W_{G}: w^{-1} \alpha>0 \forall \alpha \in \Delta^{+}\left(\mathfrak{m}_{\mathbb{C}}, \mathfrak{b}_{\mathbb{C}}\right)\right\} .
$$

As above, let $d=2 n+1$, where $d=\operatorname{dim} X$. Then for $k=0, \ldots, 2 n$ let $H^{k}\left(\mathfrak{n}, V_{\rho}\right)$ be the cohomology of $\mathfrak{n}$ with coefficients in $V_{\rho}$. Then $H^{k}\left(\mathfrak{n}, V_{\rho}\right)$ is an $M A$ module. In our case, the theorem of Kostant [Ko] states:

Proposition 6.1. In the sense of MA-modules one has

$$
H^{k}\left(\mathfrak{n} ; V_{\rho}\right) \cong \sum_{\substack{w \in W^{1} \\ l(w)=k}} V_{\rho(w)},
$$

where $V_{\rho(w)}$ is the $M A$ module of highest weight $w\left(\Lambda(\rho)+\rho_{G}\right)-\rho_{G}$.

Proof. See for example [BW, Theorem III.3], [Wa2, Theorem 2.5.1.3].

For $w \in W^{1}$ let $\sigma_{\rho, w}$ be the representation of $M$ with highest weight

$$
\Lambda\left(\sigma_{\rho, w}\right):=\left.w\left(\Lambda(\rho)+\rho_{G}\right)\right|_{\mathfrak{b}_{\mathbb{C}}}-\rho_{M}
$$

and let $\lambda_{\rho, w} \in \mathbb{C}$ such that

$$
\left.w\left(\Lambda(\rho)+\rho_{G}\right)\right|_{\mathfrak{a}_{\mathbb{C}}}=\lambda_{\rho, w} e_{1} .
$$

We have $\rho_{G}\left(H_{1}\right)=n$. Thus if for $w \in W_{1}, \ell(w)=k$, we regard $V_{\rho(w)}$ as subset of $H^{k}\left(\mathfrak{n} ; V_{\rho}\right)$ via the isomorphism in Proposition 6.1, then one has

$$
\left(\Lambda^{k} \operatorname{Ad}^{*} \otimes \rho\right)(a(t)) v=e^{\left(\lambda_{\rho, w}-n\right) t} v, \quad \forall v \in V_{\rho(w)} .
$$

For $k=0, \ldots n$ let $\sigma_{\rho, k}$ be the representation of $M$ with highest weight

$$
\Lambda\left(\sigma_{\rho, k}\right):=\left(\rho_{1}+1\right) e_{2}+\cdots+\left(\rho_{k}+1\right) e_{k+1}+\rho_{k+2} e_{k+2}+\cdots+\rho_{n+1} e_{n+1} .
$$

For $k=0, \ldots n$ let

$$
\lambda_{\rho, k}:=\rho_{k+1}+n-k
$$


For $k=n+1, \ldots, 2 n$ let $\lambda_{\rho, k}:=-\lambda_{\rho, 2 n-k}$ and let $\sigma_{\rho, k}:=w_{0} \sigma_{\rho, 2 n-k}$. Finally, let

$$
\left\{\begin{array}{llll}
\lambda_{\rho, n}^{+}:=\lambda_{\rho, n} ; & \lambda_{\rho, n}^{-}:=-\lambda_{\rho, n}, & \sigma_{\rho, n}^{+}:=\sigma_{\rho, n} ; \quad \sigma_{\rho, n}^{-}:=w_{0} \sigma_{\rho, n} & \text { if } \rho_{n+1} \geq 0 \\
\lambda_{\rho, n}^{+}:=-\lambda_{\rho, n} ; & \lambda_{\rho, n}^{-}:=\lambda_{\rho, n}, & \sigma_{\rho, n}^{+}:=w_{0} \sigma_{\rho, n} ; \quad \sigma_{\rho, n}^{-}:=\sigma_{\rho, n} & \text { if } \rho_{n+1}<0 .
\end{array}\right.
$$

Then by the computations in [BW, Chapter VI.3] one has

$$
\begin{aligned}
& \left\{\left(\lambda_{\rho, w}, \sigma_{\rho, w}, l(w)\right): w \in W^{1}\right\} \\
= & \left\{\left(\lambda_{\rho, k}, \sigma_{\rho, k}, k\right): k=0, \ldots, n-1\right\} \sqcup\left\{\left(\lambda_{\rho, n}^{+}, \sigma_{\rho, n}^{+}, n\right),\left(\lambda_{\rho, n}^{-}, \sigma_{\rho, n}^{-}, n\right)\right\} \\
& \sqcup\left\{\left(\lambda_{\rho, k}, \sigma_{\rho, k}, k\right): k=n+1, \ldots, 2 n\right\},
\end{aligned}
$$

see [MP1, section 2.8]. We equip $V_{\rho}$ with the admissible inner product from section 3 and we let $\mathcal{H}^{p}\left(\mathfrak{n} ; V_{\rho}\right)$ be the space of harmonic forms in $\Lambda^{p} \mathfrak{n}^{*} \otimes V_{\rho}$. Then by the finitedimensional Hodge theorem, each cohomology class in $H^{p}\left(\mathfrak{n} ; V_{\rho}\right)$ has a unique harmonic representative in $\mathcal{H}^{p}\left(\mathfrak{n} ; V_{\rho}\right)$. Moreover, $\mathcal{H}^{p}\left(\mathfrak{n} ; V_{\rho}\right)$ is an $M A$-invariant subspace which is $M A$-equivalent to $H^{p}\left(\mathfrak{n} ; V_{\rho}\right)$. We put

$$
\mathcal{H}^{n}\left(\mathfrak{n} ; V_{\rho}\right)_{ \pm}:=\mathcal{H}^{n}\left(\mathfrak{n} ; V_{\rho}\right) \cap\left(\Lambda^{n} \mathfrak{n}^{*} \otimes V_{\rho}\right)_{\sigma_{\rho, n}^{ \pm}} .
$$

Then, if we use the notation (5.2), the following corollary holds.

Corollary 6.2. Let $k \neq n$ and let $\Phi \in \mathcal{H}^{k}\left(\mathfrak{n} ; V_{\rho}\right)$. Then the form $\Phi_{\lambda}$ is closed if and only if $\lambda=-\lambda_{\rho, k}$. Moreover, for every $t \in \mathbb{R}$ one has $\iota_{a(t)^{*}} \Phi_{-\lambda_{\rho, k}}=\Phi$.

For $\Phi \in \mathcal{H}^{n}\left(\mathfrak{n} ; V_{\rho}\right)_{ \pm}$, the form $\Phi_{\lambda}$ is closed if and only if $\lambda=-\lambda_{\rho, n}^{ \pm}$. Moreover, for every $t \in \mathbb{R}$ one has $\iota_{a(t) *} \Phi_{-\lambda_{\rho, n}^{ \pm}}^{ \pm}=\Phi$.

Proof. This follows immediately from Lemma 5.1, Lemma 5.2 and from equation (6.4).

For $\Phi \in \mathcal{H}^{p}\left(\mathfrak{n} ; V_{\rho}\right)$, we can also construct a form in $C^{\infty}\left(\Gamma_{N} \backslash G ; \nu_{p+1}(\rho)\right)$ as follows. Let

$$
*: \Lambda^{p}\left((\mathfrak{n} \oplus \mathfrak{a})^{*}\right) \otimes V_{\rho} \rightarrow \Lambda^{d-p}\left((\mathfrak{n} \oplus \mathfrak{a})^{*}\right) \otimes V_{\rho}
$$

be the Hodge star operator which is defined as the Hodge star operator on $\Lambda^{p}\left((\mathfrak{n} \oplus \mathfrak{a})^{*}\right)$ tensored with the identiy on $V_{\rho}$. We let $\#: V_{\rho} \cong V_{\rho}^{*}$ be the isomorphism induced by the inner product on $V_{\rho}$, which extends to an isomorphism

$$
\#: \Lambda^{p}\left((\mathfrak{n} \oplus \mathfrak{a})^{*}\right) \otimes V_{\rho} \rightarrow \Lambda^{p}\left((\mathfrak{n} \oplus \mathfrak{a})^{*}\right) \otimes V_{\rho}^{*} .
$$

The operators \# and $*$ are canonically defined on $C^{\infty}\left(\Gamma_{N} \backslash G ; \nu_{p}(\rho)\right)$ and the codifferential

$$
\delta: C^{\infty}\left(\Gamma_{N} \backslash G, \nu_{p}(\rho)\right) \rightarrow C^{\infty}\left(\Gamma_{N} \backslash G, \nu_{p-1}(\rho)\right)
$$

is given by

$$
\delta:=(-1)^{d p+d+1} * \circ \#^{-1} d \# \circ * .
$$

This is the formal adjoint of $d$ on $L^{2}\left(\Gamma_{N} \backslash G, \nu_{p}(\rho)\right.$, where the latter space is defined in the same way as the space $C^{\infty}\left(\Gamma_{N} \backslash G, \nu_{p}(\rho)\right)$ using the induced metric on $F_{P}$ and the admissible inner product on $V_{\rho}$. We let $*_{1}: \Lambda^{p}\left(\mathfrak{n}^{*}\right) \otimes V_{\rho} \rightarrow \Lambda^{2 n-p}\left(\mathfrak{n}^{*}\right) \otimes V_{\rho}$ be the restriction of $*$. Now for $\Phi \in \Lambda^{p} \mathfrak{n}^{*} \otimes V_{\rho}$ we define an $E_{\rho}$-valued $p+1$-form $\tilde{\Phi}_{\lambda} \in C^{\infty}\left(\Gamma_{N} \backslash G, \nu_{p+1}(\rho)\right)$ by

$$
\tilde{\Phi}_{\lambda}(n a(t) k):=e^{(\lambda+(d-1) / 2) t} \nu_{p}(\rho)\left(k^{-1}\right)\left(d H_{1} \wedge \Phi\right),
$$


where $n \in N, a(t) \in A, k \in K$.

Lemma 6.3. For $\Phi \in \mathcal{H}^{k}\left(\mathfrak{n} ; V_{\rho}\right), k \neq n$ and $\lambda \in \mathbb{C}$ the form $\tilde{\Phi}_{\lambda}$ is closed. It is coclosed if and only if $\lambda=\lambda_{\rho, k}$. For $\Phi \in \mathcal{H}^{n}\left(\mathfrak{n} ; V_{\rho}\right)_{ \pm}$and $\lambda \in \mathbb{C}$ the form $\tilde{\Phi}_{\lambda}$ is closed. It is coclosed if and only if $\lambda= \pm \lambda_{\rho, n}^{ \pm}$. Finally, one has $\iota_{a}^{*} \tilde{\Phi}_{\lambda}=0$ for each $a \in A$.

Proof. Since $\Phi$ is closed, the form $\tilde{\Phi}_{\lambda}$ is obviously closed for each $\lambda$. In the notation (5.2) we have $\# \circ * \tilde{\Phi}_{\lambda}=\left(\# \circ *_{1} \Phi\right)_{\lambda}$ and since $\Phi \in \mathcal{H}^{k}\left(\mathfrak{n} ; V_{\rho}\right)$, one has $\# *_{1} \Phi \in \mathcal{H}^{2 n-k}\left(\mathfrak{n} ; V_{\rho^{*}}\right)$. Since the inner product on $V_{\rho}$ is admissible, for $v \in V_{\rho}$ and $a(t) \in A$ with $\rho(a(t)) v=e^{\lambda t} v$ one has $\rho^{*}(a(t))(\# v)=e^{-\lambda t} \# v$. On the other hand, $\Lambda^{p} \operatorname{Ad}^{*}(a(t))$ acts as $e^{-p t}$ on $\Lambda^{p} \mathfrak{n}^{*}$ for every $t \in \mathbb{R}$. Since $A$ is abelian, it follows that if for $\Phi \in \Lambda^{p} \mathfrak{n}^{*} \otimes V_{\rho}$ there exists $\lambda \in \mathbb{R}$ with

$$
\left(\Lambda^{p} \operatorname{Ad}^{*} \otimes \rho\right)(a(t)) \Phi=e^{\lambda t} \Phi, \quad t \in \mathbb{R}
$$

then

$$
\left(\Lambda^{2 n-p} \operatorname{Ad} \otimes \rho^{*}\right)(a(t))\left(\# \circ *_{1} \Phi\right)=e^{(-2 n-\lambda) t}\left(\# \circ *_{1} \Phi\right), \quad t \in \mathbb{R} .
$$

Thus the first statements of the Lemma follow from Lemma 5.1 and equation (6.4). Since $\iota_{a}^{*} d H_{1}=0$, the last statement is obvious.

For $Y \in(0, \infty)$ we let $F_{P}(Y)$ be as in (2.14). We denote the restriction of the bundle $E_{\rho}$ from section 3 to $F_{P}(Y)$ by $E_{\rho}$ too. Then the space of smooth $E_{\rho}$-valued $p$-forms on $F_{P}(Y)$ is canonically isomorphic to the spaces $C^{\infty}\left(\Gamma_{N} \backslash N A[Y] K, \nu_{p}(\rho)\right)$ which is defined analogously to the spaces above. We let $L^{2}\left(\Gamma_{N} \backslash N A[Y] K, \nu_{p}(\rho)\right)$ be the space of all measurable functions $f: \Gamma_{N} \backslash N A[Y] K \rightarrow \Lambda^{p}(\mathfrak{n} \oplus \mathfrak{a})^{*} \otimes V_{\rho}$ which satisfy

$$
f\left(\Gamma_{N} n a k\right)=\nu_{p}(\rho)(k)^{-1} f(n a)
$$

for allmost all $\Gamma_{N} n a k \in \Gamma_{N} \backslash N A[Y] K, a \in A[Y], n \in N, k \in K$ and whose norm

$$
\|f\|_{L^{2}\left(F_{P}(Y) ; \Lambda^{p} E_{\rho}\right)}^{2}=\int_{\log Y}^{\infty} \int_{\Gamma_{N} \backslash N} e^{-(d-1) t}\|f(\bar{n} a(t))\|^{2} d_{\Gamma_{N} \backslash N}(\bar{n}) d t
$$

is finite. Here the norm in $\Lambda^{p}(\mathfrak{n} \oplus \mathfrak{a})^{*} \otimes V_{\rho}$ is the norm induced by the metric on $\mathfrak{n} \oplus \mathfrak{a} \subset \mathfrak{g}$ and the admissible inner product on $V_{\rho}$. It follows that for $\Phi \in \Lambda^{p} \mathfrak{n}^{*} \otimes V_{\rho}$ and $\lambda \in \mathbb{R}, \lambda<0$, the form $\tilde{\Phi}_{\lambda}$, restricted to $F_{P}(Y)$, where it defines an $E_{\rho}$-valued $p$-forms, is square-integrable. More precisely, if $\Phi, \Psi \in \Lambda^{p} \mathfrak{n}^{*} \otimes V_{\rho}$ and if $\lambda<0$, one has

$$
\begin{aligned}
\left\langle\tilde{\Phi}_{\lambda}, \tilde{\Psi}_{\lambda}\right\rangle_{L^{2}\left(F_{P}(Y) ; \Lambda^{p} E_{\rho}\right)} & =\langle\Phi, \Psi\rangle \operatorname{vol}\left(\Gamma_{N} \backslash N\right) \int_{\log Y}^{\infty} e^{2 t \lambda} d t \\
& =\langle\Phi, \Psi\rangle \operatorname{vol}\left(\Gamma_{N} \backslash N\right) \frac{Y^{2 \lambda}}{2|\lambda|}
\end{aligned}
$$

If $\rho \neq \rho_{\theta}$, then $\lambda_{p, \rho}<0$ for $p>n$ and $\lambda_{n, \rho}^{-}<0$. Thus using Lemma 6.3 and the preceding equation, for $p \geq n+1$ one can define an orthonormal set of harmonic $E_{\rho}$-valued $p$-forms on $F_{P}(Y)$ which satisfy relative boundary conditions and which are square-integrable. It will be shown in Lemma 16.1 that one can obtains an orthornormal basis of the associated cohomology in this way. 


\section{EISENSTEIN SERIES}

We let $X=\Gamma \backslash \widetilde{X}$ be the hyperbolic manifold of section 2.7, we let $\rho \in \operatorname{Rep}(G)$ and we let $E_{\rho}$ be the flat vector bundle over $X$ as in section 3. In Corollary 6.2, we have constructed closed $E_{\rho}$ valued $p$-forms on the cusps of the manifold. The next step in Harder's approach [Ha] now is to lift these forms to $E_{\rho}$ valued $p$-forms on $X$ by averaging them over the cosets $\Gamma \cap N_{P_{i}} \backslash \Gamma$ corresponding to the cusps of $X$. Here one uses the theory of Eisenstein series to treat the analytic properties of the obtained infinite sums. Therefore, in this section we shall briefly review the theory of Eisenstein series.

For $\sigma \in \hat{M}, P_{i} \in \mathfrak{P}_{\Gamma}$ we define a representation $\sigma_{P_{i}}$ of $M_{P_{i}}$ by

$$
\sigma_{P_{i}}\left(m_{P_{i}}\right):=\sigma\left(k_{P_{i}}^{-1} m_{P_{i}} k_{P_{i}}\right), \quad m_{P_{i}} \in M_{P_{i}} .
$$

Let $\nu \in \hat{K}$ and $\sigma \in \hat{M}$ such that $[\nu: \sigma] \neq 0$. Then we let $\mathcal{E}_{P_{i}}(\sigma, \nu)$ be the set of all continuous functions $\Phi$ on $G$ which are left-invariant under $N_{P_{i}} A_{P_{i}}$ such that for all $x \in G$ the function $m_{P_{i}} \mapsto \Phi_{P_{i}}\left(m_{P_{i}} x\right)$ belongs to $L^{2}\left(M_{P_{i}}, \sigma_{P_{i}}\right)$, the $\sigma_{P_{i}}$-isotypical component of the right regular representation of $M_{P_{i}}$, and such that for all $x \in G$ the function $k \mapsto \Phi_{P_{i}}(x k)$ belongs to the $\nu$-isotypical component of the right regular representation of $K$. The space $\mathcal{E}_{P_{i}}(\sigma, \nu)$ is finite-dimensional and one can show that $\operatorname{dim}\left(\mathcal{E}_{P_{i}}(\sigma, \nu)\right)=\operatorname{dim}(\sigma) \operatorname{dim}(\nu)$.

We define an inner product on $\mathcal{E}_{P_{i}}(\sigma, \nu)$ as follows. Any element of $\mathcal{E}_{P_{i}}(\sigma, \nu)$ can be identified canonically with a function on $K$. For $\Phi, \Psi \in \mathcal{E}_{P_{i}}(\sigma, \nu)$ put

$$
\langle\Phi, \Psi\rangle:=\operatorname{vol}\left(\Gamma \cap N_{P_{i}} \backslash N_{P_{i}}\right) \int_{K} \Phi(k) \bar{\Psi}(k) d k .
$$

Define the Hilbert space $\mathcal{E}_{P_{i}}(\sigma)$ by

$$
\mathcal{E}_{P_{i}}(\sigma):=\bigoplus_{\substack{\nu \in \hat{K} \\[\nu: \sigma] \neq 0}} \mathcal{E}_{P_{i}}(\sigma, \nu)
$$

Let

$$
\mathcal{E}(\sigma, \nu):=\bigoplus_{P_{i} \in \mathfrak{P}_{\Gamma}} \mathcal{E}_{P_{i}}(\sigma, \nu) ; \quad \mathcal{E}(\sigma):=\bigoplus_{P_{i} \in \mathfrak{P}_{\Gamma}} \mathcal{E}_{P_{i}}(\sigma)
$$

For $\sigma \in \hat{M}, P_{i} \in \mathfrak{P}_{\Gamma}$ and $\lambda \in \mathbb{C}$ we let $\mathcal{E}_{P_{i}}(\sigma, \lambda)$ be the $L^{2}(K)$-closure of all continuous functions $\Psi$ on $G$ which satisfy

$$
\Psi\left(n_{P_{i}} a_{P_{i}}(t) x\right)=e^{(\lambda+n) t} \Psi(x), \quad t \in \mathbb{R}, n_{P_{i}} \in N_{P_{i}}, x \in G
$$

and for which the functions $m_{P_{i}} \mapsto \Psi\left(m_{P_{i}} x\right), x \in G$, belong to $L^{2}\left(M_{P_{i}} ; \sigma_{P_{i}}\right)$. We let

$$
\mathcal{E}(\sigma, \lambda):=\bigoplus_{i=1}^{\kappa(\Gamma)} \mathcal{E}_{P_{i}}(\sigma, \lambda)
$$

and we let $G$ act on $\mathcal{E}_{P_{i}}(\sigma, \lambda)$ resp. $\mathcal{E}(\sigma, \lambda)$ by the right regular representation. For $\Phi \in \mathcal{E}_{P_{i}}(\sigma)$ we define a function $\Phi_{\lambda}$ on $G$ by $\Phi_{\lambda}\left(n_{P_{i}} a_{P_{i}}(t) k\right):=e^{(n+\lambda) t} \Phi(k), n_{P_{i}} \in N_{P_{i}}$, $a_{P_{i}} \in A_{P_{i}}, k \in K$. Then the assignment $\Phi \mapsto \Phi_{\lambda}, \Phi \in \mathcal{E}_{P_{i}}(\sigma)$, is an isomorphism between $\mathcal{E}_{P_{i}}(\sigma)$ and $\mathcal{E}_{P_{i}}(\sigma, \lambda)$. Finally, $G$ acts on $\mathcal{E}(\sigma, \lambda)$ by the right regular representation and it 
is easy to see that $\mathcal{E}(\sigma, \lambda)$ with this $G$ action is equivalent to $\kappa(\Gamma) \operatorname{dim}(\sigma)$ copies of the principal series representation $\pi_{\sigma,-i \lambda}$, where the latter is parametrized as in (2.6), see for example [Pf1, section 4].

Now we let $\rho \in \operatorname{Rep}(G)$ be a finite-dimensional irreducible representation of $G$ on $V_{\rho}$. Let $P_{i} \in \mathfrak{P}_{\Gamma}$. Then the results of the previous sections 4 , 5] and 6 carry over to the parabolic subgroup $P_{i}$, where we use the fixed $k_{P_{i}}$-s in $K$ to identify each $P_{i}=k_{P_{i}} P k_{P_{i}}^{-1}$ with $P$. In particular, for $\sigma \in \hat{M}$, we shall denote by $\left(\Lambda^{p} \mathfrak{n}_{P_{i}}^{*} \otimes V_{\rho}\right)_{\sigma}$ the $\sigma_{P_{i}}$-isotypical component of the representation $\Lambda^{p} \mathrm{Ad}^{*} \otimes \rho$ of $M_{P_{i}}$ on $\left(\Lambda^{p} \mathfrak{n}_{P_{i}}^{*} \otimes V_{\rho}\right)$. For $\Phi_{P_{i}} \in \Lambda^{p_{\mathfrak{n}}} \mathfrak{n}_{P_{i}} \otimes V_{\rho}$ we define the element $\Phi_{P_{i}, \lambda}:=\left(\Phi_{P_{i}}\right)_{\lambda} \in C^{\infty}\left(\left(\Gamma \cap N_{P_{i}}\right) \backslash G, \nu_{p}(\rho)\right)$ as in (5.2). The complex $C^{*}\left(\mathfrak{g}, K ; \mathcal{E}_{P_{i}}(\check{\sigma}, \lambda) \otimes V_{\rho}\right)$ is a subcomplex of $C^{*}\left(\mathfrak{g}, K ; C^{\infty}\left(\left(\Gamma \cap N_{P_{i}}\right) \backslash G\right) \otimes V_{\rho}\right)$. Moreover, we have the following Lemma.

Lemma 7.1. Let $\Phi_{P_{i}} \in\left(\Lambda^{p} \mathfrak{n}_{P_{i}}^{*} \otimes V_{\rho}\right)_{\sigma}$. Then with respect to the isomorphism (4.5), one has

$$
\Phi_{P_{i}, \lambda} \in C^{p}\left(\mathfrak{g}, K ; \mathcal{E}_{P_{i}}(\check{\sigma}, \lambda) \otimes V_{\rho}\right),
$$

where $\check{\sigma}$ is the contragredient representation of $\sigma$.

Proof. For $X \in \Lambda^{p}(\mathfrak{g} / \mathfrak{k})$ and $g \in G, n_{P_{i}} \in N_{P_{i}}, a_{P_{i}}(t) \in A_{P_{i}}$ the function $\Phi_{P_{i}, \lambda ; X}$, defined as in the end of section 4 obviously satisfies

$$
\Phi_{P_{i}, \lambda ; X}\left(n_{P_{i}} a_{P_{i}}(t) g\right)=e^{(\lambda+n) t} \Phi_{P_{i}, \lambda ; X}(g) .
$$

To show that the function $m_{P_{i}} \mapsto \Phi_{P_{i}, \lambda ; X}\left(m_{P_{i}} g\right)$ belongs to $L^{2}\left(M_{P_{i}} ; \check{\sigma}_{P_{i}}\right) \otimes V_{\rho}$, one just uses the Schur orthogonality relations [Kn2, Corollary 4.10]. by

For $\Phi_{P_{i}} \in\left(\Lambda^{p} \mathfrak{n}_{P_{i}}^{*} \otimes V_{\rho}\right)_{\sigma}$ one defines the Eisenstein series $E\left(\Phi_{P_{i}}: \lambda: x\right) \in C^{\infty}\left(\Gamma \backslash G, \nu_{p}(\rho)\right)$

$$
E\left(\Phi_{P_{i}}: \lambda\right)(x):=\sum_{\gamma \in\left(\Gamma \cap N_{P_{i}}\right) \backslash \Gamma} \Phi_{P_{i}, \lambda}(\gamma g) ; \quad x=\Gamma g .
$$

By Lemma 7.1, under the isomorphism (4.5), $\Phi_{P_{i}, \lambda}$ can be written as a finite sum

$$
\Phi_{P_{i}, \lambda}=\sum_{j} \omega_{j} \otimes \Psi_{P_{i}, \lambda}^{j} \otimes v_{j}
$$

where $\omega_{j} \in \Lambda^{p}(\mathfrak{g} / \mathfrak{k})^{*}, \Psi_{P_{i}}^{j} \in \mathcal{E}_{P_{i}}(\check{\sigma})$ resp. $\Psi_{P_{i}, \lambda}^{j} \in \mathcal{E}_{P_{i}}(\check{\sigma}, \lambda)$, and $v_{j} \in V_{\rho}$. Thus the main results about the "usual" scalar valued Eisenstein series, due to Selberg [Se], Langlands [La], Harish-Chandra [HC] and others, continue to hold for the vector-valued Eisenstein series defined in (7.3) with the obvious modifications.

We shall now briefly review them. On $\Gamma \backslash G \times\{\lambda \in \mathbb{C}: \operatorname{Re}(\lambda)>n\}$ the series (7.3) is absolutely and locally uniformly convergent. As a function of $\lambda$, it has a meromorphic continuation to $\mathbb{C}$ with only finitely many poles in the strip $0<\operatorname{Re}(\lambda) \leq n$ which are located on $(0, n]$ and it has no poles on the line $\operatorname{Re}(\lambda)=0$. 
For $P_{i}, P_{j} \in \mathfrak{P}_{\Gamma}, \Phi_{P_{i}} \in\left(\Lambda^{p} \mathfrak{n}_{P_{i}}^{*} \otimes V_{\rho}\right)_{\sigma}$ and $g \in G$ let

$$
E_{P_{j}}\left(\Phi_{P_{i}}: \lambda\right)(g):=\frac{1}{\operatorname{vol}\left(\Gamma \cap N_{P_{j}} \backslash N_{P_{j}}\right)} \int_{\Gamma \cap N_{P_{j}} \backslash N_{P_{j}}} E\left(\Phi_{P_{i}}: \lambda\right)(n g) d n
$$

be the constant term of $E\left(\Phi_{P_{i}}: \lambda\right)$ along $P_{j}$. Then there exists a meromorphic function

$$
\underline{C}_{P_{i} \mid P_{j}}(\sigma: \lambda): C^{*}\left(\mathfrak{g}, K ; \mathcal{E}_{P_{i}}(\sigma: \lambda) \otimes V_{\rho}\right) \rightarrow C^{*}\left(\mathfrak{g}, K ; \mathcal{E}_{P_{j}}\left(w_{0} \sigma:-\lambda\right) \otimes V_{\rho}\right)
$$

such that for $P_{i}, P_{j} \in \mathfrak{P}_{\Gamma}$ one has

$$
E_{P_{j}}\left(\Phi_{P_{i}}: \lambda\right)=\delta_{i, j} \Phi_{P_{i}, \lambda}+\underline{C}_{P_{i} \mid P_{j}}(\sigma: \lambda) \Phi_{P_{i}, \lambda} .
$$

Furthermore, let

$$
\underline{\mathbf{C}}(\sigma, \lambda): \bigoplus_{P_{i} \in \mathfrak{P}_{\Gamma}} C^{*}\left(\mathfrak{g}, K ; \mathcal{E}_{P_{i}}(\sigma: \lambda) \otimes V_{\rho}\right) \rightarrow \bigoplus_{P_{i} \in \mathfrak{P}_{\Gamma}} C^{*}\left(\mathfrak{g}, K ; \mathcal{E}_{P_{i}}\left(w_{0} \sigma:-\lambda\right) \otimes V_{\rho}\right)
$$

be the map built from the maps $\underline{C}_{P_{i} \mid P_{j}}(\sigma, \lambda)$. Then one has

$$
\underline{\mathbf{C}}\left(w_{0} \sigma, \lambda\right) \underline{\mathbf{C}}(\sigma,-\lambda)=\mathrm{Id} ; \quad \underline{\mathbf{C}}(\sigma, \lambda)^{*}=\underline{\mathbf{C}}\left(w_{0} \sigma, \bar{\lambda}\right) \text {. }
$$

Let $P_{i}, P_{j} \in \mathfrak{P}_{\Gamma}$ and let $\Phi_{P_{i}} \in\left(\Lambda^{p} \mathfrak{n}_{P_{i}}^{*} \otimes V_{\rho}\right)_{\sigma}$. Then for $\underline{C}_{P_{i} \mid P_{j}}(\sigma: \lambda) \Phi_{P_{i}, \lambda}$, regarded as an element of $C^{\infty}\left(\Gamma \cap N_{P_{j}} \backslash G, \nu_{p}(\rho)\right)$, and for $a \in A_{P_{j}}$ one has

$$
\iota_{a}^{*}\left(\underline{C}_{P_{i} \mid P_{j}}(\sigma: \lambda) \Phi_{P_{i}, \lambda}\right) \in\left(\Lambda^{p} \mathfrak{n}_{P_{j}}^{*} \otimes V_{\rho}\right)_{w_{0} \sigma},
$$

where $\iota_{a}^{*}$ is as in section 5. This follows easily from the usual properties of the constant term matrix and the above constructions.

Remark 7.2. The maps $\underline{C}_{P_{i} \mid P_{j}}(\sigma, \lambda)$ are of course constructed out of the usual constant-term matrices $C_{P_{i} \mid P_{j}}$ of Eisenstein series, used in [MP2], [MP5]. However, by the isomorphism from Lemma [7.1, $\underline{C}_{P_{i} \mid P_{j}}(\sigma, \lambda)$ is in fact constructed out of the matrix $C_{P_{i} \mid P_{j}}(\check{\sigma}, \lambda)$ used in these articles.

\section{BASEs of COHOMOlOGy ClASSES on CERTAin Flat BUNDles USing Eisenstein SERIES}

We can now now give explicit bases of the De Rham cohomology groups $H^{*}\left(X ; E_{\rho}\right)$ of $X$ with coefficients in the flat vector bundles $E_{\rho}$ for those $\rho \in \operatorname{Rep}(G)$ which satisfy $\rho \neq \rho_{\theta}$ using the approach of Harder and Schwermer.

We let $X$ be as in section 2.7. and we let $Y \geq Y_{0}$, where $Y_{0}$ is as in section 2.7. Then we can restrict $E_{\rho}$ to $X(Y)$ and to the boundary $\partial X(Y)$ of $X(Y)$. We shall denote the corresponding De Rham (or equivalently singular) cohomology groups by $H^{*}\left(X(Y) ; E_{\rho}\right)$ and $H^{*}\left(\partial X(Y) ; E_{\rho}\right)$. By $H^{*}\left(X(Y), \partial X(Y) ; E_{\rho}\right)$ we denote the relative cohomology groups corresponding to the pair of spaces $X(Y)$ and $\partial X(Y)$ and the bundle $E_{\rho}$. We have a long exact sequence

$$
\begin{aligned}
& \cdots \rightarrow H^{p}\left(X(Y), \partial X(Y) ; E_{\rho}\right) \rightarrow H^{p}\left(X(Y) ; E_{\rho}\right) \rightarrow H^{p}\left(\partial X(Y) ; E_{\rho}\right) \\
& \rightarrow H^{p+1}\left(X(Y) ; E_{\rho}\right) \rightarrow \ldots
\end{aligned}
$$


The cohomology groups $H^{p}\left(X(Y) ; E_{\rho}\right)$ are of course independent of $Y$ and are just equal to the De Rham cohomology groups $H^{p}\left(X ; E_{\rho}\right)$ or can be written as $H^{p}\left(\bar{X} ; E_{\rho}\right)$, where $\bar{X}$ denotes the Borel-Serre compactification of $X$. However, since later on we shall work with the truncated manifolds $X(Y)$, we shall use them here too. Let $H_{!}^{p}\left(X(Y) ; E_{\rho}\right)$ denote the image of $H^{p}\left(X(Y), \partial X(Y) ; E_{\rho}\right)$ in $H^{p}\left(X(Y) ; E_{\rho}\right)$ in the long exact sequence above.

Let $H_{(2)}^{p}\left(X ; E_{\rho}\right)$ denote the $L^{2}$-cohomology groups of $X$ with coefficients in $E_{\rho}$. By definition, these are the cohomology groups of the subcomplex of the $E_{\rho^{-}}$-valued De Rham complex on $X$ which is formed by the smooth $E_{\rho}$-valued differential forms on $X$ that are square integrable and whose exterior derivative is also square integrable. Then the following Proposition holds.

Proposition 8.1. Let $\rho \in \operatorname{Rep}(G), \rho \neq \rho_{\theta}$. Then one has $H_{(2)}^{*}\left(X ; E_{\rho}\right)=0$.

Proof. The group $G$ acts on $L^{2}(\Gamma \backslash G)$ by the right regular representation $\pi_{\Gamma}$ and one denotes by $L^{2}(\Gamma \backslash G)^{\infty}$ the corresponding smooth $K$-finite vectors. Then by a result of Borel [Bo2, Theorem 3.5] one has $H_{(2)}^{*}\left(X ; E_{\rho}\right)=H^{*}\left(\mathfrak{g}, K ; V_{\rho} \otimes L^{2}(\Gamma \backslash G)^{\infty}\right)$. According to the spectral decomposition of $\pi_{\Gamma}$, this cohomology splits into a finite direct sum of $(\mathfrak{g}, K)$ cohomology spaces with coefficients either in irreducible unitary representations of $G$ or in direct integrals of tempered principal series representations of $G,[\mathrm{BG}$, Theorem 5.3]. Using the condition $\rho \neq \rho_{\theta}$, it follows that the cohomology of the first type is zero by [BW], Proposition II. 6.12] and that the cohomology of the second type is zero by [BC, Theorem 3.6].

Remark 8.2. Proposition 8.1 can also be proved as follows. Let $\Delta_{p}(\rho)$ be the flat HodgeLaplace operator acting on the smooth compactly supported $E_{\rho}$-valued $p$-forms on $X$. Since $X$ is complete, this operator is essentially self adjoint [Ch] and its closure will be denoted by the same symbol. It folllows from the results of [MP2, section 7], [MP5, section 5] that the spectrum $\sigma\left(\Delta_{p}(\rho)\right)$ of $\Delta_{p}(\rho)$ satisfies $\sigma\left(\Delta_{p}(\rho)\right) \subseteq\left[\frac{1}{4}, \infty\right)$. More precisely, by the theory of Eisenstein series applied in [MP2], [MP5] to the present case, the continuous spectrum of $\Delta_{p}(\rho)$ consists of the intervals $[\rho(\Omega)-c(\sigma), \infty)$, each occuring with multiplicity $\kappa(\Gamma)$, where $\sigma \in \hat{M}$ is such that $\left[\nu_{p}(\rho): \sigma\right] \neq 0$, where $c(\sigma)$ is as in (2.10) and where $\rho(\Omega)$ is the Casimir eigenvalue of $\Omega$. Thus by [MP2, Lemma 7.1] and the condition $\rho \neq \rho_{\theta}$, the continuous spectrum of $\Delta_{p}(\rho)$ lies in $[1 / 4, \infty)$. The same holds for the discrete spectrum by [MP2, Lemma 7.3], [MP5, Corollary 5.2]. Since $\Delta_{p}(\rho)$ is essentially selfadjoint, by [BL, Lemma 3.8] the complex formed by the smooth $E_{\rho}$-valued differential forms on $X$ with compact support has a unique ideal boundary condition. By [BL, Theorem 3.5] the cohomology of this Hilbert complex coincides with the cohomology $H_{(2)}^{*}\left(X ; E_{\rho}\right)$. Since the $\Delta_{p}(\rho)$ are the Laplacians of this Hilbert complex, Proposition 8.1 follows therefore from [BL, Theorem 2.4, Corollary 2.5].

By the next Lemma the De Rham cohomlogy of $X$ with coefficients in $E_{\rho}$ is determined by its restriction to the boundary if $\rho \neq \rho_{\theta}$. 
Lemma 8.3. Let $\rho \in \hat{G}$ and assume that $\rho \neq \rho_{\theta}$. Then one has $H_{!}^{p}\left(X(Y) ; E_{\rho}\right)=0$ for each $p$ or, in other words, the restriction map $H^{p}\left(X(Y) ; E_{\rho}\right) \rightarrow H^{p}\left(\partial X(Y) ; E_{\rho}\right)$ is injective for each $p$.

Proof. Let $H_{c}^{*}\left(X ; E_{\rho}\right)$ denote the compactly supported De Rham cohomology of $X$ with coefficients in $E_{\rho}$ and let $H_{!}^{*}\left(X ; E_{\rho}\right)$ be the interior cohomology, i.e. the image of $H_{c}^{*}\left(X ; E_{\rho}\right)$ in $H^{*}\left(X ; E_{\rho}\right)$. Then we can identify $H_{!}^{*}\left(X(Y) ; E_{\rho}\right)$ with $H_{!}^{*}\left(X ; E_{\rho}\right)$. Moreover, Proposition 8.1 implies that $H_{!}^{*}\left(X ; E_{\rho}\right)=0$.

For $P_{i} \in \mathfrak{P}_{\Gamma}$, we define the spaces $\mathcal{H}^{k}\left(\mathfrak{n}_{P_{i}} ; V_{\rho}\right), \mathcal{H}^{n}\left(\mathfrak{n}_{P_{i}} ; V_{\rho}\right)_{ \pm}$as in section 6. We also let the $\lambda_{\rho, k} \in \mathbb{R}$ be as in (6.7).

Proposition 8.4. Let $\rho \in \hat{G}$ and assume that $\rho \neq \rho_{\theta}$. For $k>n, P_{i} \in \mathfrak{P}_{\Gamma}$ and $\Phi_{P_{i}} \in$ $\mathcal{H}^{k}\left(\mathfrak{n}_{P_{i}} ; V_{\rho}\right)$ the Eisenstein series $E\left(\Phi_{P_{i}}: \lambda\right)$ is holomorphic in $\lambda=-\lambda_{\rho, k}$. Moreover, $E\left(\Phi_{P_{i}}:-\lambda_{\rho, k}\right)$ is closed. For $\Phi_{P_{i}} \in \mathcal{H}^{n}\left(\mathfrak{n}_{P_{i}} ; V_{\rho}\right)_{-}$, the Eisenstein series $E\left(\Phi_{P_{i}}: \lambda\right)$ is holomorphic in $\lambda=-\lambda_{\rho, n}^{-}$and $E\left(\Phi_{P_{i}}:-\lambda_{\rho, n}^{-}\right)$is closed.

Proof. We can assume without loss of generality that $\mathfrak{n}_{P_{i}}=\mathfrak{n}$. If $w_{0}$ is the non-trivial element of the restricted Weyl group $W(A)$, then for $\rho \neq \rho_{\theta}$ one has $\sigma_{\rho, w} \neq w_{0} \sigma_{\rho, w}$ for each $w \in W^{1}$ by (2.7), (2.8) and (6.7). Thus by [KS, Propositions 49, 53], there exists no complementary series corresponding to $\sigma_{\rho, w}$, i.e. in our parametrization no representation $\pi_{\sigma_{\rho, w}, i \lambda}, \lambda \in \mathbb{R}$, is unitarizable. As it is well known, this implies that the Eisenstein-Series $E(\Phi: \lambda)$ is holomorphic on $\{\lambda \in \mathbb{C}: \operatorname{Re}(\lambda)>0\}:$ If $\lambda_{0}$ was a pole of $E(\Phi: \lambda)$ lying in this set, it would be a simple pole with $\lambda_{0} \in(0, n]$ and by the spectral decomposition of the right regular representation $\pi_{\Gamma}$ of $G$ on $L^{2}(\Gamma \backslash G)$, [La], Wa, the corresponding residue of the Eisenstein series would establish $\pi_{\sigma_{\rho, w},-i \lambda_{0}}$ as a direct summand of $\pi_{\Gamma}$, which contradicts the absence of complementary series. If $\rho \neq \rho_{\theta}$, then by (2.7) and (6.7), for $k>n$ one has $\lambda_{\rho, k}<0$ and one has $\lambda_{\rho, n}^{-}<0$. Thus the statement about the holomorphy of the corresponding Eisenstein series follows.

Assume that $k>n$. If $\Phi \in \mathcal{H}^{k}\left(\mathfrak{n} ; V_{\rho}\right)$, then by (6.4) one has $\left(\Lambda^{k} \operatorname{Ad}^{*} \otimes \rho\right)\left(H_{1}\right) \Phi=$ $\left(\lambda_{\rho, k}-n\right) \Phi$ and thus by Lemma 5.1 the form $\Phi_{\lambda}$ is closed at $\lambda=-\lambda_{\rho, k}$. This implies that also $E\left(\Phi:-\lambda_{\rho, k}\right)$ is closed, [Sch, 4.3(4)]. For $k=n$ one argues in the same way.

In the next three propositions, we can now describe the cohomology groups $H^{*}\left(X ; E_{\rho}\right)$ completely and we can also give explicity canonical bases. We would like to emphasize again that these propositions are essentially elaborations of the ideas of Harder [Ha] and Schwermer Sch. For $Y \geq Y_{0}$ we shall use the identification

$$
H^{*}\left(\partial X(Y) ; E_{\rho}\right) \cong \bigoplus_{P_{i} \in \mathfrak{P}_{\Gamma}} H^{k}\left(\Gamma \cap N_{P_{i}} \backslash N_{P_{i}} ; E_{\rho}\right) \cong \bigoplus_{P_{i} \in \mathfrak{P}_{\Gamma}} \mathcal{H}^{k}\left(\mathfrak{n}_{P_{i}} ; V_{\rho}\right),
$$

where the second isomorphism, induced by the map (5.1) is the isomorphism of van Est vE]. Moreover, by $\iota_{k}^{*}(Y)$ we shall denote the restriction map

$$
\iota_{k}^{*}(Y): H^{k}\left(X(Y) ; E_{\rho}\right) \rightarrow H^{k}\left(\partial X(Y) ; E_{\rho}\right)
$$

in cohomology. To ease notation, we shall denote the restriction $\left.\omega\right|_{X(Y)}$ of an $E_{\rho^{-} \text {-valued }}$ differential form $\omega$ on $X$ to an $E_{\rho}$-valued differential form on $X(Y)$ by the same symbol $\omega$. 
Proposition 8.5. Let $\rho \in \operatorname{Rep}(G)$ and assume that $\rho \neq \rho_{\theta}$. Let $n<k \leq 2 n$ and let $Y \geq Y_{0}$. Then $\iota_{k}^{*}(Y)$ is an isomorphism. More explicitly, for $\Phi \in \bigoplus_{P_{i} \in \mathfrak{P}_{\Gamma}} \mathcal{H}^{k}\left(\mathfrak{n}_{P_{i}} ; V_{\rho}\right)$ the Eisenstein series $E\left(\Phi:-\lambda_{\rho, k}\right)$ is a non-trivial cohomology class with $\iota_{k}^{*}(Y) E\left(\Phi:-\lambda_{\rho, k}\right)=$ $\Phi$.

Proof. We can assume that there exists a $P_{i} \in \mathfrak{P}_{\Gamma}$ with $\Phi \in \mathcal{H}^{k}\left(\mathfrak{n}_{P_{i}} ; V_{\rho}\right)$. According to the previous section we shall write $\Phi=\Phi_{P_{i}}$. By Proposition 8.4, the Eisenstein series $E\left(\Phi_{P_{i}}: \lambda\right)$ has no pole in $\lambda=-\lambda_{\rho, k}$ and $E\left(\Phi_{P_{i}}:-\lambda_{\rho, k}\right)$ is closed. Thus by [Ha, page 152], [Sch, Satz 1.10] also the constant term $E_{P_{j}}\left(\Phi_{P_{i}}:-\lambda_{\rho, k}\right)$ along $P_{j}$ of $E\left(\Phi_{P_{i}}:-\lambda_{\rho, k}\right)$ is closed and the restriction of $E\left(\Phi_{P_{i}}:-\lambda_{\rho, k}\right)$ to the boundary component associated to $P_{j}$ in the decomposition (2.15) is cohomologous to the restriction of $E_{P_{j}}\left(\Phi_{P_{i}}:-\lambda_{\rho, k}\right)$ to the boundary component associated to $P_{j}$. By (7.4) one has

$$
E_{P_{j}}\left(\Phi_{P_{i}}:-\lambda_{\rho, k}\right)=\delta_{i, j} \Phi_{P_{i},-\lambda_{\rho, k}}+\underline{C}_{P_{i} \mid P_{j}}\left(\sigma_{\rho, k},-\lambda_{\rho, k}\right) \Phi_{P_{i},-\lambda_{\rho, k}} .
$$

Since $k>n$ and since $\Phi_{P_{i}}$ is harmonic, one has $\Phi_{P_{i}} \in\left(\Lambda^{k} \mathfrak{n}_{P_{i}}^{*} \otimes V_{\rho}\right)_{\sigma_{\rho, k}}$ by (6.7). Thus by (7.6), for $a \in A_{P_{j}}$ one has

$$
\iota_{a}^{*}\left(\underline{C}_{P_{i} \mid P_{j}}\left(\sigma_{\rho, k},-\lambda_{\rho, k}\right) \Phi_{P_{i},-\lambda_{\rho, k}}\right) \in\left(\Lambda^{k} \mathfrak{n}_{P_{j}}^{*} \otimes V_{\rho}\right)_{w_{0} \sigma_{\rho, k}} .
$$

However, as in the proof of Proposition 8.4, by the condition $\rho \neq \rho_{\theta}$ one has $\sigma_{\rho, k} \neq w_{0} \sigma_{\rho, k}$. Thus, since $k>n$ one has $\mathcal{H}^{k}\left(\mathfrak{n}_{P_{j}}^{*} ; V_{\rho}\right) \cap\left(\Lambda^{p} \mathfrak{n}_{P_{j}}^{*} \otimes V_{\rho}\right)_{w_{0} \sigma_{\rho, k}}=0$ by (6.7). Applying Corollary (6.2), one obtains $\iota_{k}^{*}(Y) E\left(\Phi_{P_{i}}:-\lambda_{\rho, k}\right)=\Phi_{P_{i}}$ and the proposition follows from Lemma 8.3 .

Next we prove the vanishing of the $E_{\rho}$-valued De Rham cohomology in degree $k<n$.

Proposition 8.6. Let $\rho \in \operatorname{Rep}(G)$ and assume that $\rho \neq \rho_{\theta}$. Then for $k<n$, one has $H^{k}\left(X(Y) ; E_{\rho}\right)=0$.

Proof. By Lemma 8.3, it suffices to show that for $k<n$ the map $\iota_{k}^{*}(Y)$ is zero. We let $E_{\rho}^{*}$ be the dual bundle of $E_{\rho}$. As in the case of trivial coefficients, we have a canonical pairing

$$
B: H^{k}\left(\partial X(Y) ; E_{\rho}\right) \otimes H^{2 n-k}\left(\partial X(Y) ; E_{\rho}^{*}\right) \rightarrow \mathbb{C}, \quad B(\omega, \eta):=\int_{\partial X(Y)}(\omega \wedge \eta)(x) d x,
$$

where $\omega \wedge \eta$ is the ordinary $2 n$-form on $\partial X(Y)$ defined as in [Mü4, page 729]. Also, as in the case of trivial coefficients, the classical Hodge theorem implies that the pairing $B$ is non-degenerate. By Stoke's theorem, for $\omega \in H^{k}\left(X(Y) ; E_{\rho}\right)$ and $\eta \in H^{2 n-k}\left(X(Y) ; E_{\rho}^{*}\right)$ one has $B\left(\iota_{k}^{*}(Y) \omega, \iota_{k}^{*}(Y) \eta\right)=0$. Let $\check{\rho}$ be the contragredient representation of $\rho$. Then the dual bundle $E_{\rho}^{*}$ is canonically isomorphic to $E_{\check{\rho}}$. By [GW, section 3.2.5], for $n$ odd $\check{\rho}$ is equivalent to $\rho$ and for $n$ even $\check{\rho}$ is equivalent to $\rho_{\theta}$. In particular, if $\rho$ is not equivalent to $\rho_{\theta}$, then also $\check{\rho}$ is not equivalent to $(\check{\rho})_{\theta}$ and thus by the previous Proposition 8.5, for $k>n$ the map $\iota_{k}^{*}(Y): H^{2 n-k}\left(X(Y) ; E_{\rho}^{*}\right) \rightarrow H^{2 n-k}\left(\partial X(Y) ; E_{\rho}^{*}\right)$ is surjective. Therefore, for $k<n$ the map $\iota_{k}^{*}(Y): H^{k}\left(X(Y) ; E_{\rho}\right) \rightarrow H^{k}\left(\partial X(Y) ; E_{\rho}\right)$ must be the zero map.

It remains to consider the cohomology $H^{n}\left(X(Y) ; E_{\rho}\right)$. We will denote the projection of $\Psi \in \bigoplus_{P_{i} \in \mathfrak{P}_{\Gamma}} \Lambda^{n}\left(\mathfrak{n}_{P_{i}}\right)^{*} \otimes V_{\rho}$ to the subspace $\bigoplus_{P_{i} \in \mathfrak{P}_{\Gamma}} \mathcal{H}^{n}\left(\mathfrak{n}_{P_{i}} ; V_{\rho}\right)_{+}$by $[\Psi]_{+}$. If we use that 
$\lambda_{\rho, n}^{+}=-\lambda_{\rho, n}^{-}$, it follows easily from Lemma 5.2 and from equation (6.4) that for $P_{i}, P_{j} \in \mathfrak{P}_{\Gamma}$, $\Phi \in \mathcal{H}^{n}\left(\mathfrak{n}_{P_{i}} ; V_{\rho}\right)_{-}$and $a \in A_{P_{j}}$ the form $\left[\iota_{a}^{*}\left(\underline{C}_{P_{i} \mid P_{j}}\left(\sigma_{\rho, n}^{-}:-\lambda_{\rho, n}^{-}\right) \Phi_{-\lambda_{\rho, n}^{-}}\right)\right]_{+} \in \mathcal{H}^{n}\left(\mathfrak{n}_{P_{j}} ; V_{\rho}\right)_{+}$ does not depend on $a$. Therefore, we obtain a well defined map from $\bigoplus_{P_{i} \in \mathfrak{P}_{\Gamma}} \mathcal{H}^{n}\left(\mathfrak{n}_{P_{i}} ; V_{\rho}\right)_{-}$ to $\bigoplus_{P_{i} \in \mathfrak{P}_{\Gamma}} \mathcal{H}^{n}\left(\mathfrak{n}_{P_{i}} ; V_{\rho}\right)_{+}$which we shall denote by

$$
\Phi \mapsto\left[\underline{\mathbf{C}}\left(\sigma_{\rho, n}^{-},-\lambda_{\rho, n}^{-}\right) \Phi\right]_{+}
$$

for brevity. Then we have the following proposition.

Proposition 8.7. Let $\rho \in \operatorname{Rep}(G), \rho \neq \rho_{\theta}$. Then one has

$$
\operatorname{dim} H^{n}\left(X(Y) ; E_{\rho}\right)=\frac{1}{2} \operatorname{dim} H^{n}\left(\partial X(Y) ; E_{\rho}\right) .
$$

More explicitly, for $\Phi \in \bigoplus_{P_{i} \in \mathfrak{P}_{\Gamma}} \mathcal{H}^{n}\left(\mathfrak{n}_{P_{i}} ; V_{\rho}\right)_{-}$one has

$$
i_{n}^{*}(Y) E\left(\Phi:-\lambda_{\rho, n}^{-}\right)=\Phi+\left[\underline{\mathbf{C}}\left(\sigma_{\rho, n}^{-},-\lambda_{\rho, n}^{-}\right) \Phi\right]_{+} \cdot
$$

and if $\left\{\Phi_{l}\right\}$ is a basis of $\bigoplus_{P_{i} \in \mathfrak{P}_{\Gamma}} \mathcal{H}^{n}\left(\mathfrak{n}_{P_{i}} ; V_{\rho}\right)_{-}$, then a basis of $H^{n}\left(X(Y) ; E_{\rho}\right)$ is given by the $E\left(\Phi_{l}:-\lambda_{\rho, n}^{-}\right)$.

Proof. One has

$$
\chi\left(X(Y) ; E_{\rho}\right)=\frac{1}{2} \chi\left(\partial X(Y) ; E_{\rho}\right)=\frac{1}{2} \operatorname{dim}\left(V_{\rho}\right) \chi(\partial X(Y))=0,
$$

where the last equality follows from the fact that $\partial X(Y)$ is a disjoint union of finitely many tori. Thus by Proposition 8.5 and Proposition 8.6 we conclude

$$
\begin{aligned}
(-1)^{n+1} \operatorname{dim} H^{n}\left(X(Y) ; E_{\rho}\right) & =\sum_{k=n+1}^{2 n}(-1)^{k} \operatorname{dim} H^{k}\left(X(Y) ; E_{\rho}\right) \\
& =\sum_{k=n+1}^{2 n}(-1)^{k} \operatorname{dim} H^{k}\left(\partial X(Y) ; E_{\rho}\right) .
\end{aligned}
$$

If we apply the isomorphism (8.1), Proposition 6.1 and (6.7), we see immediately that we have $\operatorname{dim} H^{k}\left(\partial X(Y) ; E_{\rho}\right)=\operatorname{dim} H^{2 n-k}\left(\partial X(Y) ; E_{\rho}\right)$ for each $k$ (let us recall that $\rho$ is not necessarily self-dual). Thus from $\chi(\partial(X(Y)))=0$ we conclude

$$
\sum_{k=n+1}^{2 n}(-1)^{k} \operatorname{dim} H^{k}\left(\partial X(Y) ; E_{\rho}\right)=\frac{1}{2}(-1)^{n+1} \operatorname{dim} H^{n}\left(\partial X(Y) ; E_{\rho}\right) .
$$

Combining the last two equations, we obtain (8.3).

To prove (8.4), one can proceed exactly as in the proof of Proposition [8.5, using that in degree $n$ one has $w_{0} \sigma_{\rho, n}^{-}=\sigma_{\rho, n}^{+}$. Since $\operatorname{dim} \mathcal{H}^{n}\left(\mathfrak{n}_{P_{i}} ; V_{\rho}\right)_{-}=\operatorname{dim} \mathcal{H}^{n}\left(\mathfrak{n}_{P_{i}} ; V_{\rho}\right)_{+}$and since the sum in (8.4) is direct, the $E\left(\Phi_{l}:-\lambda_{\rho, n}^{-}\right)$form a basis of $H^{n}\left(X(Y) ; E_{\rho}\right)$ by (8.3) and (8.4). 
At the end of this section, we remark that Harder's construction of Eisenstein cohomology classes also applies if $\rho \neq \rho_{\theta}$. The difference then is that the Eisenstein series might have a pole at the point where one has to evaluate it, since Proposition 8.4 critically uses that $\rho \neq \rho_{\theta}$. If the Eisenstein series has a pole, one has to take its residue [Ha] and the resulting cohomology class is actually a square-integrable cohomology class - Proposition 8.1 holds only if $\rho \neq \rho_{\theta}$. Finally, as pointed out by Harder, the cohomology classes defined by Eisenstein series in Propositions 8.5 and 8.7 are always harmonic in the sense that they are smooth $E_{\rho}$-valued $p$-forms on $X$ which are annihilated by the flat Hodge Laplacian. In the present parametrization, this can just be read off from [MP5, (3.8)] and Kuga's formula. However, in our case the Eisenstein cohomology classes are never square integrable.

\section{The Reidemeister torsion With RESPECt to Eisenstein COHOMOLOGy}

CLASSES

In this section we define the Reidemeister torsion $\tau_{\text {Eis }}\left(\bar{X} ; E_{\rho}\right), \rho \neq \rho_{\theta}$ of $X$ with coefficients in $E_{\rho}$ with respect to the basis in the cohomology of the previous section.

We will firstly briefly review the definition of the Reidemeister torsion of compact manifolds, possibly with boundary. For further details, we refer to the paper [Mil] of Milnor. We follow [M̈̈4, section 1]. Firstly, we recall the definition of the Reidemeister torsion of a cochain complex. We remark that we take the opposite sign than [Mü4] since we work with cochain complexes. For a finite-dimensional real vector space $V$ of dimension $d>0$ we use the common notation $\operatorname{det} V:=\Lambda^{d} V$. A non-zero element of $\operatorname{det} V$ is called a volume element. If $L$ is a one-dimensional real vector space, we let $L^{-1}:=L^{*}$ and for $l \in L, l \neq 0$, we let $l^{-1} \in L^{*}$ be the unique element defined by $l^{-1}(l)=1$. If

$$
C^{*}: 0 \rightarrow C^{0} \stackrel{d_{0}}{\longrightarrow} C^{1} \rightarrow \cdots \rightarrow C^{n-1} \stackrel{d_{n-1}}{\longrightarrow} C^{n} \rightarrow 0
$$

is a cochain complex of finite-dimensional non-zero real vector spaces $C^{q}$ and if $H^{q}$ are its cohomology groups, we let

$$
\left(\operatorname{det} C^{*}\right)^{-1}:=\bigotimes_{q}\left(\operatorname{det} C^{q}\right)^{(-1)^{q+1}} ; \quad \operatorname{det} H^{*}:=\bigotimes_{q}\left(\operatorname{det} H^{q}\right)^{(-1)^{q}} .
$$

Then the Reidemeister torsion $\tau\left(C^{*}\right)$ is an invariant associated to the cochain complex $C^{*}$ which is defined as follows. Let $b_{q}:=\operatorname{dim} d_{q}\left(C^{q}\right), h_{q}:=\operatorname{dim} H^{q}$. Let $\theta_{q} \in \Lambda^{b_{q}} C^{q}$ such that $d_{q}\left(\theta^{q}\right) \neq 0$. Let $Z^{q}:=\operatorname{Ker}\left(d_{q}\right)$ and let $\iota_{q}: Z_{q} \rightarrow C_{q}$ be the inclusion. Let $\mu_{q} \in \operatorname{det} H^{q}$, $\mu_{q} \neq 0$, and let $\nu_{q} \in \Lambda^{h_{q}}\left(Z^{q}\right)$ such that $\pi\left(\nu_{q}\right)=\mu_{q}$, where $\pi: Z^{q} \rightarrow H^{q}$ is the canonical projection. Then $\omega_{q}:=d_{q-1}\left(\theta_{q-1}\right) \wedge \theta_{q} \wedge \iota_{q}\left(\nu_{q}\right)$ is a nonzero element of $\operatorname{det} C^{q}$ and one defines

$$
\tau\left(C^{*}\right):=\bigotimes_{q} \omega_{q}^{(-1)^{q+1}} \otimes \bigotimes_{q} \mu_{q}^{(-1)^{q}} \in\left(\operatorname{det} C^{*}\right)^{-1} \otimes \operatorname{det} H^{*} .
$$

It is easy to see that $\tau\left(C^{*}\right)$ does not depend on the choices of $\theta_{q}, \mu_{q}$ and $\nu_{q}$. 
If for each $q$ bases $\mathcal{A}_{q}:=\left\{\omega_{q, 1}, \ldots, \omega_{\left.q, m_{q}\right)}\right\}$ of $C^{q}, m_{q}:=\operatorname{dim} C_{q}$, and $\mathcal{B}_{q}:=\left\{\eta_{q, 1}, \ldots, \eta_{q, h_{q}}\right\}$ of $H^{q}$ are given, one obtaines non-zero elements

$$
\omega:=\bigotimes_{q}\left(\omega_{q, 1} \wedge \cdots \wedge \omega_{q, m_{q}}\right)^{(-1)^{q+1}} ; \quad \eta:=\bigotimes_{q}\left(\eta_{q, 1} \wedge \cdots \wedge \eta_{q, h_{q}}\right)^{(-1)^{q}}
$$

of $\left(\operatorname{det} C^{*}\right)^{-1} /\{ \pm 1\}$ resp. $\operatorname{det} H^{*} /\{ \pm 1\}$. The element $\omega$ determines an identification $\left(\operatorname{det} C^{*}\right)^{-1} \otimes \operatorname{det} H^{*} \cong \operatorname{det} H^{*} /\{ \pm 1\}$ and $\eta$ determines an identification $\operatorname{det} H^{*} /\{ \pm 1\} \cong \mathbb{R}^{+}$. Thus if $\omega$ is fixed, one can associate to $\tau\left(C^{*}\right)$ an element $\tau\left(C^{*}, \omega\right) \in \operatorname{det} H^{*} /\{ \pm 1\}$ and if $\omega$ and $\eta$ are fixed, one can associate to $\tau\left(C^{*}\right)$ an element $\tau\left(C^{*}, \omega, \mu\right) \in(0, \infty)$.

Now let $M$ be a compact smooth manifold with boundary $\partial M$. Assume that $\partial M$ is a disjoint union $\partial M=\partial M_{1} \sqcup \partial M_{2}$. We do not exclude that $\partial M, \partial M_{1}$ or $\partial M_{2}$ are empty. Let $\tilde{M}$ be the universal covering of $M$ with a fixed base-point. Then the fundamental group $\pi_{1}[M]$ of $M$ acts on $\tilde{M}$ by deck transformations. If $\rho$ is a finite-dimensional representation of $\pi_{1}[M]$ on a real or complex vector space $V_{\rho}$, we let $F_{\rho}:=\tilde{M} \times_{\pi_{1}[M]} V_{\rho}$ be the associated flat vector bundle over $M$; every flat vector bundle is obtained in this way. The representation $\rho$ is called unimodular if $|\operatorname{det}(\rho)(\gamma)|=1$ for all $\gamma \in \pi_{1}[M]$.

Let $\mathcal{K}$ be a smooth triangulation of $M$ containing a subcomplex $\mathcal{K}_{1}$ triangulating $\partial M_{1}$. Let $\widetilde{\mathcal{K}}$ be the lift of $\mathcal{K}$ to a triangulation of $\tilde{M}$ and let $\widetilde{\mathcal{K}}_{1}$ be the corresponding lift of $\mathcal{K}_{1}$; then $\widetilde{\mathcal{K}}_{1}$ is a subcomplex of $\widetilde{\mathcal{K}}$. Let $C^{q}(\widetilde{\mathcal{K}})$ resp. $C^{q}\left(\widetilde{\mathcal{K}}_{1}\right)$ be the real vector space generated by the $q$-cochains of $\widetilde{\mathcal{K}}$ resp. $\widetilde{\mathcal{K}}_{1}$. The real group algebra $\mathbb{R}\left[\pi_{1}(M)\right]$ acts on the spaces $C^{q}(\widetilde{\mathcal{K}})$ resp. $C^{q}\left(\widetilde{\mathcal{K}}_{1}\right)$ by deck transformations and one defines

$$
C^{q}\left(\mathcal{K} ; F_{\rho}\right):=C^{q}(\widetilde{\mathcal{K}}) \otimes_{\mathbb{R}\left[\pi_{1}(M)\right]} V_{\rho} ; \quad C^{q}\left(\mathcal{K}_{1} ; F_{\rho}\right):=C^{q}\left(\widetilde{\mathcal{K}}_{1}\right) \otimes_{\mathbb{R}\left[\pi_{1}(M)\right]} V_{\rho}
$$

The operators $d_{q}: C^{q}(\widetilde{\mathcal{K}}) \rightarrow C^{q+1}(\widetilde{\mathcal{K}})$ and $d_{q}: C^{q}\left(\widetilde{\mathcal{K}}_{1}\right) \rightarrow C^{q+1}\left(\widetilde{\mathcal{K}}_{1}\right)$ induce coboundary operators $d_{q}: C^{q}\left(\mathcal{K} ; F_{\rho}\right) \rightarrow C^{q+1}\left(\mathcal{K} ; F_{\rho}\right)$ and $d_{q}: C^{q}\left(\mathcal{K}_{1} ; F_{\rho}\right) \rightarrow C^{q+1}\left(\mathcal{K}_{1} ; F_{\rho}\right)$ and one obtains cochain complexs $C^{*}\left(\mathcal{K} ; F_{\rho}\right)$ and $C^{*}\left(\mathcal{K}_{1} ; F_{\rho}\right)$. The second complex is a subcomplex of the first one and thus the spaces

$$
C^{q}\left(\mathcal{K}, \mathcal{K}_{1} ; F_{\rho}\right):=C^{q}\left(\mathcal{K} ; F_{\rho}\right) / C^{q}\left(\mathcal{K}_{1} ; F_{\rho}\right)
$$

also form a cochain complex which one denotes by $C^{*}\left(\mathcal{K}, \mathcal{K}_{1} ; F_{\rho}\right)$. Let $H^{*}\left(\mathcal{K} ; F_{\rho}\right)$ resp. $H^{*}\left(\mathcal{K}, \mathcal{K}_{1} ; F_{\rho}\right)$ denote the cohomology groups of $C^{*}\left(\mathcal{K} ; F_{\rho}\right)$ resp. $C^{*}\left(\mathcal{K}, \mathcal{K}_{1} ; F_{\rho}\right)$. Then these groups are canonically isomorphic to the singular cohomology groups $H^{*}\left(M ; F_{\rho}\right)$ resp. the relative singular cohomology groups $H^{*}\left(M, \partial M_{1} ; F_{\rho}\right)$ of $M \operatorname{resp} .\left(M, \partial M_{1}\right)$ with coefficients in $F_{\rho}$, Wh.

Fix a basis $x_{1}, \ldots, x_{m}, m=\operatorname{dim}\left(V_{\rho}\right)$, of $V_{\rho}$ and consider the associated element $\theta:=$ $x_{1} \wedge \cdots \wedge x_{m} \in \operatorname{det}\left(V_{\rho}\right) /\{ \pm 1\}$. Fix an embedding of $\mathcal{K}$ into $\tilde{\mathcal{K}}$. Then tensoring the $x_{i}$ with the $q$-cochains of $\mathcal{K}$ resp. $\mathcal{K}-\mathcal{K}_{1}$, embedded into $\tilde{\mathcal{K}}$, one obtains a canonical family of bases of $C^{q}\left(\mathcal{K} ; F_{\rho}\right)$ and of $C^{q}\left(\mathcal{K}, \mathcal{K}_{1} ; F_{\rho}\right)$. Since $\rho$ is unimodular, the choice of embedding of $\mathcal{K}$ into $\tilde{\mathcal{K}}$ changes this basis only up to sign [M̈̈4]. Therefore, the Reidemeister torsions $\tau\left(\mathcal{K} ; F_{\rho} ; \theta\right) \in \operatorname{det} H^{*}\left(M ; F_{\rho}\right) /\{ \pm 1\} \operatorname{resp.} \tau\left(\mathcal{K}, \mathcal{K}_{1} ; F_{\rho} ; \theta\right) \in \operatorname{det} H^{*}\left(M, \partial M_{1} ; F_{\rho}\right) /\{ \pm 1\}$ are well defined. These torsions are invariant under subdivision [Mil], [M̈̈4] and are thus 
independent of the choice of the particular smooth triangulations $\mathcal{K}$ resp. $\mathcal{K}_{1}$. Thus one obtains well defined elements

$$
\tau\left(M ; F_{\rho} ; \theta\right) \in \operatorname{det} H^{*}\left(M ; F_{\rho}\right) /\{ \pm 1\} ; \tau\left(M, \partial M_{1} ; F_{\rho} ; \theta\right) \in \operatorname{det} H^{*}\left(M, \partial M_{1} ; F_{\rho}\right) /\{ \pm 1\} .
$$

The latter torsions still depend on the choice of $\theta$. However, if the Euler characteristic of the complex $C^{*}(\mathcal{K})$ resp. $C^{*}\left(\mathcal{K}, \mathcal{K}_{1}\right)$ vanishes, then they are independent of $\theta$ [Mü4 and thus they are combinatorial invariants. This is the case if $M$ is a closed odd-dimensional manifold but it will also be the case in the present paper, since here $M$ will be an odddimensional manifold whose boundary is a disjoint union of tori. If the Euler characteristic does not vanish, one has to fix a volume element $\theta$ or more generally a metric $h_{F}$ on $F_{\rho}$ resp. on $\operatorname{det} F_{\rho}$ as in [BZ], [BM1], [BM2]. In this context, we remark that in the variation formula of Brüning and Ma, the integrand of the first term on the right hand side of [BM2, (3.26)], in which the variation of $h_{F}$ is incorporated in the unimodular case, vanishes if the boundary is a union of flat tori.

If we assume that $\chi(M)=0=\chi\left(M, \partial M_{1}\right)$ and if for each $q$ bases $\mathcal{B}^{q}$ of $H^{q}\left(M ; F_{\rho}\right)$ and $\tilde{\mathcal{B}}^{q}$ of $H^{q}\left(M, \partial M_{1} ; F_{\rho}\right)$ are given and if $\mathcal{B}:=\sqcup_{q} \mathcal{B}_{q}, \widetilde{\mathcal{B}}:=\sqcup_{q} \mathcal{B}_{q}$, then by the above construction we obtain well defined elements

$$
\tau\left(M, \mathcal{B} ; F_{\rho}\right) \in(0, \infty) ; \quad \tau\left(M, \partial M_{1}, \widetilde{\mathcal{B}} ; F_{\rho}\right) \in(0, \infty) .
$$

If for each $q$ one is given another set of bases $\mathcal{B}_{q}^{\prime}$ of $H^{q}\left(M ; F_{\rho}\right)$ and if one denotes by $A_{q}$ the base change matrix from $\mathcal{B}_{q}$ to $\mathcal{B}_{q}^{\prime}$, then it is easy to see that for $\mathcal{B}^{\prime}:=\sqcup_{q} \mathcal{B}_{q}^{\prime}$ one has

$$
\tau\left(M, \mathcal{B}^{\prime} ; F_{\rho}\right)=\prod_{q}\left|\operatorname{det}\left(A_{q}\right)\right|^{(-1)^{q}} \tau\left(M, \mathcal{B} ; F_{\rho}\right) .
$$

If $g$ and $h$ are metrics on $M$ resp. $F_{\rho}$, then for each $q$ the flat Hodge-Laplace operator is defined and by the Hodge De Rham isomorphism the corresponding space of harmonic forms satisfying absolute boundary conditions is canonically isomorphic to the cohomology $H^{*}\left(M ; F_{\rho}\right)$. Thus we obtain a family of bases of $H^{*}\left(M ; F_{\rho}\right)$ which arise from $L^{2}$-orthonormal bases of harmonic forms under this isomorphisms and we shall denote by $\tau\left(M, g, h ; F_{\rho}\right) \in \mathbb{R}^{+}$the associated Reidemeister torsion. Analogously one can define $\tau\left(M, \partial M_{1}, g, h ; F_{\rho}\right)$ if one uses the Laplacians with relative boundary conditions at $\partial M_{1}$ and absolute boundary conditions at $\partial M_{2}$.

Now we can define the Reidemeister torsion of the manifold $\bar{X}$ from secion 2.7 with coefficients in the flat bundle $E_{\rho}$. We use the notations of the preceding section. For $P_{j} \in \mathfrak{P}_{\Gamma}$, the space $\Lambda^{p}\left(\mathfrak{n}_{P_{j}}\right)^{*} \otimes V_{\rho}$ carries an inner product induced by the restriction of the inner product (2.1) on $\mathfrak{g}$ to $\mathfrak{n}_{P_{j}}$ and the admissible inner product on $V_{\rho}$. We normalize this inner product by $\left(\operatorname{vol}\left(\left(\Gamma \cap N_{P_{j}}\right) \backslash N_{P_{j}}\right)\right)^{-1 / 2}$. Then the map in (5.1), which induces an isomorphism on cohomology, embeds $\Lambda^{p} \mathfrak{n}_{P_{j}}^{*} \otimes V_{\rho}$ isometrically into the space of square integrable $E_{\rho}$-valued $p$-forms on the flat torus $\left(\Gamma \cap N_{P_{j}}\right) \backslash N_{P_{j}}$ with its induced metric. For each $P_{j} \in \mathfrak{P}_{\Gamma}$ we fix an orthonormal basis $\Phi_{i, j}^{k}$ of $\mathcal{H}^{k}\left(\mathfrak{n}_{P_{j}} ; V_{\rho}\right)$ if $k>n$ and we fix an 
orthonormal basis $\Phi_{i, j}^{n}$ of $\mathcal{H}^{n}\left(\mathfrak{n}_{P_{j}} ; V_{\rho}\right)_{-}$. Then by Proposition 8.5 for $k>n$ we obtain bases

$$
\mathcal{B}_{k}:=\left\{E\left(\Phi_{i, j}^{k},-\lambda_{\rho, k}\right): j=1, \ldots, \kappa(\Gamma): i=1, \ldots, \operatorname{dim} \mathcal{H}^{k}\left(\mathfrak{n}_{P_{j}} ; V_{\rho}\right)\right\}
$$

of $H^{k}\left(X ; E_{\rho}\right)$ and by Proposition 8.7 we obtain a basis

$$
\mathcal{B}_{n}:=\left\{E\left(\Phi_{i, j}^{n},-\lambda_{\rho, n}^{-}\right): j=1, \ldots, \kappa(\Gamma): i=1, \ldots, \operatorname{dim} \mathcal{H}^{n}\left(\mathfrak{n}_{P_{j}} ; V_{\rho}\right)_{-}\right\}
$$

of $H^{n}\left(X ; E_{\rho}\right)$. Moreover, by Proposition 8.6, the cohomology groups $H^{k}\left(X ; E_{\rho}\right)$ vanish for $k<n$. If one is given another set of orthonormal bases $\Psi_{i, j}^{k}$ of each $\mathcal{H}^{k}\left(\mathfrak{n}_{P_{j}} ; V_{\rho}\right)$, then, since the assignment $\Phi \mapsto E\left(\Phi:-\lambda_{\rho, k}\right)$ is linear, the determinant of the base - change matrix $A_{k}$ from the basis $\mathcal{B}_{k}$ to the corresponding basis $\mathcal{B}_{k}^{\prime}$ has absolute value 1 . The same holds for the cohomology in degree $n$. Therefore, by (9.1) one can define the Reidemeister torsion

$$
\tau_{\text {Eis }}\left(\bar{X} ; E_{\rho}\right) \in(0, \infty)
$$

as the Reidemeister torsion of $\bar{X}$ with coefficients in $E_{\rho}$ with respect to the set of bases in the cohomology groups $H^{*}\left(\bar{X} ; E_{\rho}\right) \cong H^{*}\left(X ; E_{\rho}\right)$ formed by any set of bases $\mathcal{B}=\sqcup_{l=n}^{2 n} \mathcal{B}_{l}$ of the above form. This construction is obviously independent of the truncation parameter $Y$ which we choose to realize $\bar{X}$ as $X(Y)$, since the cohomology classes we work with now are restrictions of cohomology classes from $X$ to $X(Y)$.

\section{Regularized AND RELATIVE TRACES AND TORSIONS}

Let $X=\Gamma \backslash \tilde{X}$ be the same manifold as in section 2.7 and for $\nu \in \hat{K}$ let $E_{\nu}$ be the locally homogeneous vector bundle over $X$ as in section 3 equipped with a metric $h$ induced by the inner product on $V_{\nu}$. We let $A_{\nu}$ be the differential operator on $E_{\nu}$ which on $C^{\infty}(\Gamma \backslash G, \nu)$ acts as $-\Omega$ under the isomorphism in (3.2). By the arguments of [MP2, section 4], $A_{\nu}$ with domain the smooth compactly supported sections is bounded from below and essentially selfadjoint. Its closure will be denoted by the same symbol.

In order to allow compact perturbations of the metrics on $X$ and $E_{\rho}$ and to allow the compactly supported variation of the De Rham complex due to Vishik and Lesch, we will also need to consider operators which coincide with the operator $A_{\nu}$ only outside a compact subset of $X$. Therefore, we make the following definition, which is parallell to the definition of Müller [Mü3, Definition 5.3].

Definition 10.1. A second order elliptic differential operator $B_{\nu}$ on $C^{\infty}\left(X, E_{\nu}\right)$ is called locally homogeneous at infinity if there exists a $Y_{1} \geq Y_{0}$ and a $C\left(B_{\nu}\right) \in \mathbb{R}$ such that for all $f \in C^{\infty}\left(X, E_{\nu}\right)$ with $\left.f\right|_{X\left(Y_{1}\right)}=0$ one has $B_{\nu} f=A_{\nu} f+C\left(B_{\nu}\right) f$ and if there exist metrics $g_{1}$ on $X$ and $h_{1}$ on $E_{\nu}$ which coincide with $g$ and $h$ outside $X\left(Y_{1}\right)$ such that $B_{\nu}$ with domain the smooth compactly supported sections is symmetric with respect to the $L^{2}$-inner product induced by $g_{1}$ and $h_{1}$ and such that $B_{\nu}$ is bounded from below.

Remark 10.2. The manifold $X$ with the new metric $g_{1}$ and the bundle $E_{\nu}$ with the metric $h_{1}$ are of course just special cases of a Riemannian manifold with cusps and a bundle which is locally homogeneous at infinity in the sense of Müller [Mü3, Definitions 5.1, 5.2]. On the other hand, Definition 10.1 is slightly more general than the corresponding definition given in [Mü3, section 5], since the operators considered by Müller, who mainly studied 
index-problems, arise as squares of generalized Dirac operators, see [Mü3, Definition 5.3]. However, an inspection of the proofs shows that the results about the heat kernels of such operators obtained by Müller in section 7 of [Mü3] continue to hold for the operators $B_{\nu}$ introduced in the previous Definition 10.1.

Let $e^{-t B_{\nu}}$ be the heat semigroup of $B_{\nu}$. Then $e^{-t B_{\nu}}$ acts on $L^{2}\left(X, E_{\nu}\right)$ as an integral operator with smooth kernel

$$
K_{B_{\nu}}(t, x, y) \in C^{\infty}\left(X \times X, E_{\nu} \otimes E_{\nu}^{*}\right),
$$

where the $L^{2}$-space is taken with respect to the metrics $g_{1}$ and $h_{1}$ implicit in the definition of $B_{\nu}$. The kernel $K_{B_{\nu}}(t, x, y)$ has been studied by Müller in Mü3. For each $i=1, \ldots, \kappa(X)$ consider the complete infinite volume cusp $F_{P_{i}}:=\left(\Gamma \cap N_{P_{i}}\right) \backslash G / K$ which is equipped with the metric induced from the hyperbolic metric on $G / K$. Then $\nu$ defines a locally homogeneous vector bundle $W_{P_{i}, \nu}$ over $F_{P_{i}}$. The non-uniformity in the Gaussian estimates for $K_{B_{\nu}}$ can be controlled by the following function. Let $r_{P_{i}}$ be the function on $G$ given by

$$
r_{P_{i}}\left(n_{P_{i}} \iota_{P_{i}}(y) k\right):=y ; \quad n_{P_{i}} \in N_{P_{i}}, \iota_{P_{i}}(y) \in A_{P_{i}}, k \in K .
$$

The function $r_{P_{i}}$ obviously descends to a function on $F_{P_{i}}$. Then we fix a smooth fuction $r$ on $X$ such that $r(x)=r_{P_{i}}(x)$ if $x \in F_{P_{i}}\left(Y_{0}+1\right)$ and such that $r(x) \geq 1$ for all $x \in X$. If $C\left(B_{\nu}\right) \in \mathbb{R}$ is the constant from definition 10.1, then by the same arguments as in MP2, section 4] $-\Omega+C\left(B_{\nu}\right)$ defines an elliptic essentially selfadjoint operator $B_{P_{i}, \nu}$ which acts on the smooth section of $W_{P_{i}, \nu}$ and which is bounded from below. Let $K_{B_{P_{i}, \nu}}(t, x, y)$ be the integral kernel of $e^{-t B_{P_{i}, \nu}}$. In [Mü3], the integral kernel $K_{B_{\nu}}(t, x, y)$ was constructed by patching together the interior heat kernel of $B_{\nu}$ on the compact manifold $X\left(Y_{1}+1\right)$ with the restriction of the kernels $K_{B_{P_{i}, \nu}}(t, x, y)$ to the cusps $F_{P_{i}}\left(Y_{1}\right)$ of $X$. This construction implies the following proposition.

Proposition 10.3. For each $T>0$ there exist constants $C_{1}, C_{2}>0$ such that for all $x, y \in X$ and all $t \in(0, T]$ and $j \in\{0,1\}$ one can estimate

$$
\left\|B_{\nu}^{j} K_{B_{\nu}}(t, x, y)\right\| \leq C_{1} r(x)^{n} r(y)^{n} t^{-\frac{d}{2}-j} e^{-C_{2} \frac{d^{2}(x, y)}{t}} .
$$

Moreover, for each $T>0$ there exists a constant $C>0$ such that for each $P_{i} \in \mathfrak{P}_{\Gamma}$, all $x \in F_{P_{i}}\left(Y_{1}+1\right) \subset F_{P_{i}}\left(Y_{1}\right)$ and all $t \in(0, T]$ one has

$$
\left\|K_{B_{\nu}}(t, x, x)-K_{B_{P_{i}, \nu}}(t, x, x)\right\| \leq C e^{-\frac{\operatorname{dist}^{2}\left(x, \partial F_{P_{i}}\left(Y_{1}\right)\right)}{t}}
$$

Proof. This follows immediately from the construction of the kernel $K_{B_{\nu}}$ given by Müller in [M̈̈3, chapter 3, chapter 7] which works identically in the present situation (see remark 10.2) and for $j=0$ it is stated in [M̈̈3, Proposition 7.10]. Let us briefly outline the main steps of the argument: We may assume that $B_{\nu}$ coincides with $A_{\nu}$ outside a compact subset of $X$. Let $\tilde{A}_{\nu}$ be the lift of $A_{\nu}$ to a differential operator of Laplace type acting on the smooth sections of the bundle $\tilde{E}_{\nu}$ over $\tilde{X}$ which is defined as in section 3 and let $K_{\tilde{A}_{\nu}}$ be the integral kernel of $e^{-t \tilde{A}_{\nu}}$. Then $K_{\tilde{A}_{\nu}}$ was constructed by Donnelly for the scalar case [Do2] and, as remarked by Donnelly [Do2, page 485], this construction carries over to the 
vector valued case. We remark that Donnelly's construction is applicable in the present situation since in the present case there exist of course discrete, torsion-free subgroups $\Gamma_{1}$ of $G$ such that $\Gamma_{1} \backslash \tilde{X}$ is compact. By [Do2, page $488(\mathrm{P} 4)$ ], for each $T>0$ there exists a constant $C_{1}$ such that for all $t \in(0, T]$ and for $j \in\{0,1\}$, one has the estimate

$$
\left\|\tilde{A}_{\nu}^{j} K_{\tilde{A}_{\nu}}(t, x, y)\right\|=\left\|\frac{d^{j}}{(d t)^{j}} K_{\tilde{A}_{\nu}}(t, x, y)\right\| \leq C_{1} t^{-\frac{d}{2}-j} e^{-\frac{d^{2}(x, y)}{4 t}}, \quad x, y \in \tilde{X} .
$$

According to the isomorphism $C^{\infty}(G, \nu) \cong C^{\infty}\left(\tilde{X}, \tilde{E}_{\nu}\right)$ from section 3, we regard $K_{\tilde{A}_{\nu}}$ as a function on $G \times G$ with values in $\operatorname{End}\left(V_{\nu}\right)$. This function is invariant under the diagonal action of $G$, see [MP2, section 4]. Similarly, we regard the kernel $K_{B_{P_{i}, \nu}}$ as an $\operatorname{End}\left(V_{\nu}\right)$ valued function on $\left(\Gamma \cap N_{P_{i}}\right) \backslash G \times\left(\Gamma \cap N_{P_{i}}\right) \backslash G$ corresponding to the canonical isomorphism $C^{\infty}\left(F_{P_{i}}, E_{\nu}\right) \cong C^{\infty}\left(\left(\Gamma \cap N_{P_{i}}\right) \backslash G, \nu\right)$ which is obtained as in section 4. Then $K_{B_{P_{i}, \nu}}$ is obtained by averaging $K_{\tilde{A}_{\nu}}$ over $\Gamma \cap N_{P_{i}}$, i.e.

$$
K_{B_{P_{i} \nu}}(t, x, y)=\sum_{\gamma \in \Gamma \cap N_{P_{i}}} K_{\tilde{A}_{\nu}}(t, x, \gamma y)
$$

If $B_{\nu}=A_{\nu}$ everywhere, one of course obtains the kernel $K_{B_{\nu}}$ on $X \times X$ by averaging $K_{\tilde{A}_{\nu}}$ over the whole group $\Gamma$. By [M̈̈3, Lemma 3.20], for fixed $Y>0$ and $T>0$ there exists a constant $C>0$ such that one can estimate

$$
\sum_{\gamma \in \Gamma \cap N_{P_{i}}} e^{-\frac{d^{2}(x, \gamma y)}{4 t}} \leq C r(x)^{n} r(y)^{n} e^{-\frac{d^{2}(x, y)}{4 t}}
$$

for all $x, y \in F_{P_{i}}(Y)$ and all $t \in(0, T]$ (let us remark that the $\Gamma$ in [Mü3, chapter 3] is the present $\Gamma \cap N_{P_{i}}$ ). This implies the estimates for the kernels $K_{B_{P_{i}}}$ restricted to $F_{P_{i}}(Y) \times F_{P_{i}}(Y)$. We recall that the $F_{P_{i}}(Y)$ form the cusps of $X$. Now as in [Mü3, chapter 7], one obtains the kernel $K_{B_{\nu}}$ by patching together the kernels $K_{B_{P_{i} \nu}}, P_{i} \in \mathfrak{P}_{\Gamma}$, with the heat kernel $e_{2}$ of a compact manifold restricted to the compact part $X\left(Y_{1}+1\right)$ of $X$ using the parametrix method. A similar construction will be carried out in detail in section 13 of the present paper. Since the kernel $e_{2}$ satisfies the required estimates, one obtains the estimates in (1) as in [M̈̈3, chapter 7]. The estimate in (2) is an immediate consequence of this construction since the term $P * Q$ which can be defined as in [Mü3, chapter 7] on page 62 after (7.8) is easily seen to satisfy this estimate for $x \in F_{X}\left(Y_{1}+1\right)$ if one employs the estimate (7.7) [Mü3, chapter 7] for the function $Q$, the fact that $Q$ has uniform compact support in the first variable and the fact that the parametrix $P$ can be estimated using (7.1) in [M̈̈3, chapter 7].

We shall now review some concepts to model the heat trace of the operators just introduced. Firstly, for the operators $A_{\nu}$, in [MP2], we introduced the regularized trace of $e^{-t A_{\nu}}$ following ideas of Melrose [Me] and Park [Par]. This trace was defined as follows. Let

$$
K_{A_{\nu}}(t, x, y) \in C^{\infty}\left(X \times X, E_{\nu} \otimes E_{\nu}^{*}\right)
$$


be the integral kernel of $e^{-t A_{\nu}}$. Then by [MP2, equation 5.7] one has an asymptotic expansion

$$
\int_{X(Y)} \operatorname{Tr} K_{A_{\nu}}(t, x, x) d x=\sum_{\substack{\sigma \in \hat{M} \\[\nu: \sigma] \neq 0}}\left(\frac{\kappa(\Gamma) e^{t c(\sigma)} \log Y \operatorname{dim}(\sigma)}{\sqrt{4 \pi t}}\right)+a_{0}(t)+o(1),
$$

as $Y \rightarrow \infty$. Since on a compact manifold the trace of the heat operator is given by the integral of the pointwise fibre-trace of the heat kernel on the diagonal, we defines the regularized trace $\operatorname{Tr}_{\mathrm{reg}}\left(e^{\left.-t A_{\nu}\right)}\right)$ as the constant term $a_{0}(t)$ of the asymptotic expansion (10.1).

There is also a different way to model the definition of the heat trace, namely the concept of the relative trace, which is due to Müller [Mü3]. This concept is more convenient for our approach to the gluing formula since in this way we can avoid any interchange of limits. The idea behind the definition of the relative trace is as follows. Restricting the operator $A_{\nu}$ to the 0 -th Fourier coefficients on the cusps of the manifold, one obtains an ordinary differential operator which can be computed explicitly. Then by a result of Müller the difference of the associated heat kernels is trace class and the trace of this difference is by definition the relative trace. For a general discussion of relative traces, we refer to [Mü5].

We shall now make this definition more explicit in the present context. Let $P_{i} \in \mathfrak{P}_{\Gamma}$, let $Y_{0}$ be as in section 2.7 and let $u \in \mathbb{R}^{+}$with $u>Y_{0}$. Let $L^{2}\left([u, \infty), y^{-d} d y ; V_{\nu}\right)$ denote the space of $V_{\nu}$-valued $L^{2}$-functions on $[u, \infty)$ with respect to the measure $y^{-d} d y$ and the fixed metric on $V_{\nu}$. Then the decomposition (2.12) gives a natural inclusion $I_{P_{i}, u}$ of $L^{2}\left([u, \infty), y^{-d} d y ; V_{\nu}\right)$ into the space $L^{2}(\Gamma \backslash G, \nu)$. More explicitly, if $\chi_{u}$ is the characteristic function of the set $[u, \infty)$ then by (2.12) function

$$
I_{P_{i}, u} \phi\left(n_{P_{i}} \iota_{P_{i}}(y) k\right):=\frac{1}{\sqrt{\operatorname{vol}\left(\Gamma \cap N_{P_{i}} \backslash N_{P_{i}}\right)}} \nu\left(k^{-1}\right) \chi_{u}(y) \phi(y) ; n_{P_{i}} \in N_{P_{i}}, \iota_{P_{i}}(y) \in A_{P_{i}}, k \in K
$$

on $G$ is $\Gamma$-invariant and can therefore be regarded as an element of $L^{2}(\Gamma \backslash G, \nu)$. By (2.13) and (2.17), the assignment $\phi \mapsto I_{P_{i}, u} \phi$ embeds the space $L^{2}\left([u, \infty), y^{-d} d y ; V_{\nu}\right)$ isometrically into $L^{2}(\Gamma \backslash G, \nu)$. For $f \in L^{2}(\Gamma \backslash G, \nu)$ and $x \in \Gamma \backslash G, x=\Gamma g, g=n_{P_{i}} \iota_{P_{i}}(y) k$ let

$$
f_{P_{i}, u}(x):=\frac{1}{\operatorname{vol}\left(\Gamma \cap N_{P_{i}} \backslash N_{P_{i}}\right)} \chi_{u}(y) \int_{\Gamma \cap N_{P_{i}} \backslash N_{P}} f\left(n_{P_{i}} g\right) d n_{P_{i}} .
$$

Then $f \mapsto f_{P_{i}, u}$ is the orthogonal projection of $L^{2}(\Gamma \backslash G, \nu)$ onto $I_{P_{i}, u}\left(L^{2}\left([u, \infty), y^{-d} ; V_{\nu}\right)\right)$. Since $\Omega$ is $G$-invariant, one has $\Omega f_{P_{i}, u}=(\Omega f)_{P_{i}, u}$ for every $f \in C^{\infty}(\Gamma \backslash G, \nu)$. Thus $-\Omega$ and the embedding $I_{P_{i}, u}$ induce in a canonical way a differential operator $T_{\nu}$ on $C^{\infty}\left([u, \infty) ; V_{\nu}\right)$. The operator $T_{\nu}$ can be computed explicitly. Let $\Omega_{M_{P_{i}}}$ be the casimir element of $\mathfrak{m}_{P_{i}}$ associated to the restriction fo the normalized Killing form of $\mathfrak{g}$ to $\mathfrak{m}_{P_{i}}$ and define an endomorphism $L(\nu)$ of $V_{\nu}$ by $L(\nu):=-\left.\nu\right|_{M_{P_{i}}}\left(\Omega_{M_{P_{i}}}\right)$, where $\left.\nu\right|_{M_{P_{i}}}$ denotes the restriction of $\nu$ to $M_{P_{i}}$. Then the following Lemma holds.

Lemma 10.4. One has $T_{\nu}=-y^{2} \frac{d^{2}}{d y^{2}}+(d-2) y \frac{d}{d y}+L(\nu)$. 
Proof. Clearly, one can assume that $P_{i}=P_{0}$. For $\alpha \in \Delta^{+}\left(\mathfrak{g}_{\mathbb{C}}, \mathfrak{h}_{\mathbb{C}}\right)$ let $\mathfrak{g}_{\mathbb{C}}^{\alpha}$ be the corresponding root space. Then one can choose $X_{\alpha}$ in $\mathfrak{g}_{\mathbb{C}}^{\alpha}, X_{-\alpha} \in \mathfrak{g}_{\mathbb{C}}^{-\alpha}$ such that $B\left(X_{\alpha}, X_{-\alpha}\right)=1$, $\left[X_{\alpha}, X_{-\alpha}\right]=H_{\alpha}$, where $H_{\alpha}$ is the coroot corresponding to $\alpha$ as in section [MP2, section 2.1]. By [MP2, (2.3)] one has

$$
\sum_{\alpha \in \Delta^{+}\left(\mathfrak{g}_{\mathbb{C}}, \mathfrak{a}_{\mathbb{C}}\right)} H_{\alpha}=2 n H_{1} .
$$

Thus by the definition of $\Omega$ and $\Omega_{M}$ one has

$$
\begin{aligned}
\Omega & =\sum_{i=1}^{n+1} H_{i}^{2}+\sum_{\alpha \in \Delta^{+}\left(\mathfrak{g}_{\mathbb{C}}, \mathfrak{h}_{\mathbb{C}}\right)}\left(X_{\alpha} X_{-\alpha}+X_{-\alpha} X_{\alpha}\right) \\
& =H_{1}^{2}+\sum_{\alpha \in \Delta^{+}\left(\mathfrak{g}_{\mathbb{C}}, \mathfrak{a}_{\mathbb{C}}\right)}\left(H_{\alpha}+2 X_{-\alpha} X_{\alpha}\right)+\sum_{i=2}^{n+1} H_{i}^{2}+\sum_{\alpha \in \Delta^{+}\left(\mathfrak{m}_{\mathbb{C}}, \mathfrak{b}_{\mathbb{C}}\right)}\left(X_{\alpha} X_{-\alpha}+X_{-\alpha} X_{\alpha}\right) \\
& =H_{1}^{2}+2 n H_{1}+\Omega_{M} \bmod U\left(\mathfrak{g}_{\mathbb{C}}\right) \mathfrak{n}_{\mathbb{C}} .
\end{aligned}
$$

Now the element $H_{1}$ induces the differential operator $-y \frac{d}{d y}$ on $(0, \infty)$ under $\iota_{P}$. Since $\Omega_{M}$ is invariant under the anti-involution of $U\left(\mathfrak{m}_{\mathbb{C}}\right)$ induced by $Y \mapsto-Y, Y \in \mathfrak{m}_{\mathbb{C}}$, the proposition follows.

Consider the differential operator

$$
T_{\nu}^{0}:=-y^{2} \frac{d^{2}}{d y^{2}}+(d-2) y \frac{d}{d y}+L(\nu)
$$

acting on $C_{c}^{\infty}\left((u, \infty) ; V_{\nu}\right) \subset L^{2}\left([u, \infty), y^{-d} d y ; V_{\nu}\right)$. Its selfadjoint extension with respect to the Dirichelet boundary condition at $u$ will be denoted by $T_{\nu}^{0}$ too. First consider the operator $L(\nu)$.

Lemma 10.5. For $\sigma \in \hat{M}$ let $P_{\sigma}$ denote the orthogonal projection from $V_{\nu}$ to the $\sigma$ isotypical subspace of $\left.\nu\right|_{M}$. Then one has

$$
L(\nu)=\sum_{\substack{\sigma \in \hat{M} \\[\nu: \sigma] \neq 0}}-\left(c(\sigma)+\frac{(d-1)^{2}}{4}\right) P_{\sigma},
$$

where $c(\sigma)$ is as in (2.10).

Proof. A standard computation gives $\sigma\left(\Omega_{M}\right)=c(\sigma)+\frac{(d-1)^{2}}{4}$ and the Lemma follows.

Now consider the differential operator

$$
T^{0}:=-y^{2} \frac{d^{2}}{d y^{2}}+(d-2) y \frac{d}{d y}
$$

acting on $C_{c}^{\infty}(u, \infty) \subset L^{2}\left(\left([u, \infty), y^{-d} d y\right)\right.$. The self-adjoint extension of $T^{0}$ with Dirichelt boundary condition at $u$ will be denoted by $T^{0}$ too. 
Lemma 10.6. Let $H^{u}\left(t, y, y^{\prime}\right)$ denote the heat kernel of $T^{0}$. Then for $Y>u$ one has

$$
\int_{u}^{Y} H^{u}(t, y, y) y^{-d} d y=e^{-t \frac{(d-1)^{2}}{4}}\left(\frac{\log Y}{\sqrt{4 \pi t}}-\frac{\log u}{\sqrt{4 \pi t}}-\frac{1}{4}\right)+o(1)
$$

as $Y \rightarrow \infty$.

Proof. Consider the operator

$$
\tilde{T}^{0}:=-y^{2} \frac{d^{2}}{d y^{2}}-y \frac{d}{d y}+\frac{(d-1)^{2}}{4}
$$

with Dirichlet boundary condition at $u$ acting on $\operatorname{dom}\left(\tilde{T}^{0}\right) \subset L^{2}\left([u, \infty), y^{-1} d y\right)$. Then one has

$$
T^{0}=y^{\frac{d-1}{2}} \circ \tilde{T}^{0} \circ y^{-\frac{d-1}{2}} .
$$

Moreover, changing variables $y=e^{r}, \tilde{T}^{0}$ is equivalent to $D^{0}:=-\frac{d^{2}}{d r^{2}}+\frac{(d-1)^{2}}{4}$ where $\operatorname{dom}\left(D^{0}\right) \subset L^{2}((\log u, \infty), d r)$ and where Dirichlet boundary condition at $\log u$ for $D^{0}$ are taken. Let $p^{u}\left(t, r, r^{\prime}\right)$ denote the heat kernel of $D^{0}$. Then it is well known that

$$
p^{u}\left(t, r, r^{\prime}\right)=e^{-t \frac{(d-1)^{2}}{4}} \frac{1}{\sqrt{4 \pi t}}\left(e^{-\frac{\left(r-r^{\prime}\right)^{2}}{4 t}}-e^{-\frac{\left(r+r^{\prime}-2 \log u\right)^{2}}{4 t}}\right)
$$

see for example [CJ]. Thus one obtains

$$
H^{u}\left(t, y, y^{\prime}\right)=e^{-t \frac{(d-1)^{2}}{4}} \frac{\left(y y^{\prime}\right)^{\frac{d-1}{2}}}{\sqrt{4 \pi t}}\left(e^{-\frac{\log ^{2}\left(y^{\prime} / y\right)}{4 t}}-e^{-\frac{\left(\log \left(y y^{\prime}\right)-2 \log u\right)^{2}}{4 t}}\right)
$$

and the lemma follows from a simple computation.

For every $P_{i} \in \mathfrak{P}_{\Gamma}$ let $T_{\nu, u}^{P_{i}}$ be the operator $T_{\nu}^{0}$ regarded as an operator on $L^{2}(\Gamma \backslash G, \nu)$ via the embedding $I_{P_{i}, u}$ defined above. Then we put

$$
T_{\nu, u}:=\bigoplus_{P_{i} \in \mathfrak{P}_{\Gamma}} T_{\nu, u}^{P_{i}} .
$$

We let $K_{T_{\nu, u}}(t, x, y)$ be the integral kernel of $e^{-t T_{\nu, u}}$, where the latter heat semigroup acts on $L^{2}(\Gamma \backslash G, \nu)$ in the obvious way. If $B_{\nu}$ is locally homogeneous at infinity in the sense of definition 10.1, then we always choose the parameter $u>Y_{1}$, where $Y_{1}$ is as in Definition 10.1. We let $K_{T_{\nu, u}+C\left(B_{\nu}\right)}$ be the integral kernel of $e^{-t\left(T_{\nu, u}+C\left(B_{\nu}\right)\right)}$. Then the following proposition is due to Müller.

Proposition 10.7. Let $B_{\nu}$ be locally homogeneous at infinity with $C\left(B_{\nu}\right)$ as in Definition 10.1. Then the operator $e^{-t B_{\nu}}-e^{-t\left(T_{\nu, u}+C\left(B_{\nu}\right)\right)}$ is trace class and one has

$$
\operatorname{Tr}\left(e^{-t B_{\nu}}-e^{-t\left(T_{\nu, u}+C\left(B_{\nu}\right)\right)}\right)=\int_{X}\left(\operatorname{Tr} K_{B_{\nu}}(t, x, x)-\operatorname{Tr} K_{T_{\nu, u}+C\left(B_{\nu}\right)}(t, x, x)\right) d x,
$$

where the expression on the right is absolutely integrable. Here the integral is taken with respect to the volume induced by the metric $g_{1}$ implicit in the definition of $B_{\nu}$.

Proof. [M̈̈3, Theorem 9.1]. 
According to the previous proposition, we now define the relative trace of $e^{-t B_{\nu}}$ with respect to the parameter $u$ by

$$
\operatorname{Tr}_{\mathrm{rel}, u}\left(e^{-t B_{\nu}}\right):=\operatorname{Tr}\left(e^{-t B_{\nu}}-e^{-t\left(T_{\nu, u}+C\left(B_{\nu}\right)\right)}\right) .
$$

Then using the previous computations one can easily compare the regularized trace, introduced previously, and the relative trace:

Proposition 10.8. Let $A_{\nu}$ be as above and let $u>Y_{0}$. Then one has

$$
\operatorname{Tr}_{\mathrm{rel} ; u}\left(e^{-t A_{\nu}}\right)=\operatorname{Tr}_{\mathrm{reg}}\left(e^{-t A_{\nu}}\right)+\kappa(\Gamma) \sum_{\substack{\sigma \in \hat{M} \\[\nu: \sigma] \neq 0}} e^{t c(\sigma)} \operatorname{dim}(\sigma)\left(\frac{\log u}{\sqrt{4 \pi t}}+\frac{1}{4}\right) .
$$

Proof. By the preceding Proposition 10.7 one has

$$
\operatorname{Tr}_{\text {rel } ; u}\left(e^{-t A_{\nu}}\right)=\lim _{Y \rightarrow \infty}\left(\int_{X(Y)} \operatorname{Tr} K_{A_{\nu}}(t, x, x) d x-\int_{X(Y)} \operatorname{Tr} K_{T_{\nu, u}}(t, x, x) d x\right) .
$$

By Lemma 10.4, Lemma 10.5 and Lemma 10.6 one has

$$
\begin{aligned}
\int_{X(Y)} \operatorname{Tr} K_{T_{\nu, u}}(t, x, x) d x & =\sum_{\substack{\sigma \in \hat{M} \\
[\nu: \sigma] \neq 0}}\left(\frac{\kappa(\Gamma) e^{t c(\sigma)} \log Y \operatorname{dim}(\sigma)}{\sqrt{4 \pi t}}\right) \\
& -\kappa(\Gamma) \sum_{\substack{\sigma \in \hat{M} \\
[\nu: \sigma] \neq 0}} e^{t c(\sigma)} \operatorname{dim}(\sigma)\left(\frac{\log u}{\sqrt{4 \pi t}}+\frac{1}{4}\right)+o(1),
\end{aligned}
$$

as $Y \rightarrow \infty$. Applying (10.1), the proposition follows.

We have the following short time asymptotic expansions for the regularized and relative traces.

Proposition 10.9. One has an asymptotic expansion

$$
\operatorname{Tr}_{\mathrm{reg}}\left(e^{-t A_{\nu}}\right) \sim \sum_{j=0}^{\infty} a_{j} t^{j-\frac{d}{2}}+\sum_{j=0}^{\infty} b_{j} t^{j-\frac{1}{2}} \log t+\sum_{j=0}^{\infty} c_{j} t^{j}
$$

as $t \rightarrow 0+$. Moreover, if $B_{\nu}$ is locally homogeneous at infinity one has an asymptotic expansion

$$
\operatorname{Tr}_{\mathrm{rel} ; u}\left(e^{-t B_{\nu}}\right) \sim \sum_{j=0}^{\infty} \tilde{a}_{j} t^{j-\frac{d}{2}}+\sum_{j=0}^{\infty} \tilde{b}_{j} t^{j-\frac{1}{2}} \log t+\sum_{j=0}^{\infty} \tilde{c}_{j} t^{j}
$$

as $t \rightarrow 0+$.

Proof. The short-time asymptotic expansion of the regularized trace $\operatorname{Tr}_{\text {reg }}\left(e^{-t A_{\nu}}\right)$ was proved in [MP2, Proposition 6.9]. Applying proposition 10.8, the asymptotic expansion in (10.4) follows for the relative trace $\operatorname{Tr}_{\mathrm{rel} ; u}\left(e^{-t A_{\nu}}\right)$. For the asymptotic expansion of $\operatorname{Tr}_{\mathrm{rel} ; u}\left(e^{-t B_{\nu}}\right)$, we can assume that the constant $C\left(B_{\nu}\right)$ in definition 10.1 is zero. Let $Y_{1}>Y_{0}$ be such 
that the operators $A_{\nu}$ and $B_{\nu}$ coincide on sections supported on $X-X\left(Y_{1}\right)$. Then by Proposition 10.7 one has

$$
\begin{aligned}
\operatorname{Tr}_{\mathrm{rel} ; u}\left(e^{-t A_{\nu}}\right) & =\int_{X\left(Y_{1}+1\right)} \operatorname{Tr} K_{A_{\nu}}(t, x, x) d x-\int_{X\left(Y_{1}+1\right)} \operatorname{Tr} K_{T_{\nu, u}}(t, x, x) d x \\
& +\int_{X-X\left(Y_{1}+1\right)}\left(\operatorname{Tr} K_{A_{\nu}}(t, x, x)-\operatorname{Tr} K_{T_{\nu, u}}(t, x, x)\right) d x,
\end{aligned}
$$

Integrating the pointwise short-time asymptotic expansion of $\operatorname{Tr} K_{A_{\nu}}(t, x, x)$ ) [Gi], resp. of $\operatorname{Tr} K_{T_{\nu, u}}(t, x, x)$, over the relatively compact subset $X\left(Y_{1}+1\right)$ of $X$ and using the short time asymptotic expansion of $\operatorname{Tr}_{\mathrm{rel} ; u}\left(e^{-t A_{\nu}}\right)$, it follows that one has a short-time asymptotic expansion

$$
\begin{aligned}
& \int_{X-X\left(Y_{1}+1\right)}\left(\operatorname{Tr} K_{A_{\nu}}(t, x, x)-\operatorname{Tr} K_{T_{\nu, u}}(t, x, x)\right) d x \\
& \sim \sum_{j=0}^{\infty} a_{j} t^{j-\frac{d}{2}}+\sum_{j=0}^{\infty} b_{j} t^{j-1 / 2} \log t+\sum_{j=0}^{\infty} c_{j} t^{j}
\end{aligned}
$$

as $t \rightarrow 0+$. Now by Proposition 10.7 one has

$$
\begin{aligned}
\operatorname{Tr}_{\mathrm{rel}, u}\left(e^{-t B_{\nu}}\right) & =\int_{X\left(Y_{1}+1\right)} \operatorname{Tr} K_{B_{\nu}}(t, x, x) d x-\int_{X\left(Y_{1}+1\right)} \operatorname{Tr} K_{T_{\nu, u}}(t, x, x) d x \\
& +\int_{X-X\left(Y_{1}+1\right)}\left(\operatorname{Tr} K_{B_{\nu}}(t, x, x)-\operatorname{Tr} K_{A_{\nu}}(t, x, x)\right) d x \\
& +\int_{X-X\left(Y_{1}+1\right)}\left(\operatorname{Tr} K_{A_{\nu}}(t, x, x)-\operatorname{Tr} K_{T_{\nu, u}}(t, x, x)\right) d x,
\end{aligned}
$$

where $d x$ in the first integral of the first line stands for the volume with respect to the metric $g_{1}$ implicit in the definition of $B_{\nu}$. Again, by the pointwise short-time asymptotic expansion of the term $\operatorname{Tr} K_{B_{\nu}}(t, x, x)$ and of the term $\operatorname{Tr} K_{T_{\nu, u}}(t, x, x)$, the first integral on the right hand side of (10.6) admits a short time asymptotic expansion (without logarithmic terms). The second integral on the right hand side of (10.6) is $O\left(t^{\infty}\right)$ for $t \rightarrow 0$ by Proposition 10.3. Applying (10.5) to the third integral on the right hand side of (10.6), the proposition follows.

Now let $\rho \in \operatorname{Rep}(G)$ and let $E_{\rho}$ be the induced flat vector bundle over $X$ with the metric from section 3 and let $\Delta_{p}(\rho)$ be the associated p-th flat Hodge Laplacian as in section [3. Then by the isomorphism (3.3) and by (3.8), the bundle $E_{\rho}$ and the operator $\Delta_{p}(\rho)$ fit into the class of bundles and operators just studied. In particular, if we let $T_{p, u}^{\rho}:=T_{\nu_{p}(\rho), u}+\rho(\Omega) \operatorname{Id}, \nu_{p}(\rho)$ as in (3.6) and $\rho(\Omega) \in \mathbb{R}$ the Casimir eigenvalued of $\rho$, then the regularized trace $\operatorname{Tr}_{\mathrm{reg}}\left(e^{-t \Delta_{p}(\rho)}\right)$ and the relative trace $\operatorname{Tr}_{\mathrm{rel}, u}\left(e^{-t \Delta_{p}(\rho)}\right)=\operatorname{Tr}\left(e^{-\Delta_{p}(\rho)}-\right.$ $\left.e^{-t T_{p, u}^{\rho}}\right)$, are defined and satisfy the above properties. For brevity, according to definition 10.1 we call a second order elliptic differential operator $\Delta_{p}^{1}(\rho)$ acting on the smooth sections of $\Lambda^{p} E_{\rho}$ a compact perturbation of $\Delta_{p}(\rho)$ if $\Delta_{p}^{1}(\rho)$ is formally symmetric and bounded from 
below with respect to the $L^{2}$-space induced by some fixed compact perturbations of the hyperbolic metric on $X$ resp. the admissible metric on $E_{\rho}$ and if there exists a $Y_{1}>Y_{0}$ such that one has $\Delta_{p}^{1}(\rho) f=\Delta_{p}(\rho) f$ for all smooth sections $f$ of $E_{\rho}$ which vanish on $X\left(Y_{1}\right)$. Since $X$ is complete, $\Delta_{p}^{1}(\rho)$ is essentially selfadjoint [Ch] and its closure will be denoted by the same symbol. If $\rho \neq \rho_{\theta}$, we have the following long time estimate for the regularized and relative trace.

Proposition 10.10. Let $\rho \in \operatorname{Rep}(G)$ such that $\rho \neq \rho_{\theta}$ and let $p \in\{0, \ldots, d\}$. Then there exists a constant $C>0$ such that for each $p$ and for $t \in[1, \infty)$ one has

$$
\operatorname{Tr}_{\mathrm{reg}}\left(e^{-t \Delta_{p}(\rho)}\right) \leq C e^{-\frac{t}{4}} ; \quad \operatorname{Tr}_{\mathrm{rel}, u}\left(e^{-t \Delta_{p}(\rho)}\right) \leq C e^{-\frac{t}{4}} .
$$

Let $\Delta_{p}^{1}(\rho)$ be a compact perturbation of $\Delta_{p}(\rho)$. Then $\Delta_{p}^{1}(\rho)$ has pure point spectrum in $[0,1 / 4)$ and there exist constants $c_{1}, C_{1}>0$ such that one has

$$
\operatorname{Tr}_{\mathrm{rel}, u}\left(e^{-t \Delta_{p}^{1}(\rho)}\right)-\operatorname{dim} \operatorname{Ker}\left(\Delta_{p}^{1}(\rho)\right) \leq C_{1} e^{-c_{1} t} .
$$

for $t \geq 1$.

Proof. The estimate for the regularize trace of $e^{-t \Delta_{p}(\rho)}$ was proved in [MP2, 7.12]. By [MP2, Lemma 7.1] one has $\rho(\Omega)-c(\sigma) \geq 1 / 4$ for each $\sigma \in \hat{M}$ with $\left[\nu_{p}(\rho): \sigma\right] \neq 0$, where $\nu_{p}(\rho)$ is as in (3.6). Therefore, applying Proposition 10.8, also the estimate for the relative trace of $e^{-t \Delta_{p}(\rho)}$ follows. It was proved in [MP2], [MP5] that the spectrum $\sigma\left(\Delta_{p}(\rho)\right)$ of $\Delta_{p}(\rho)$ satisfies $\sigma\left(\Delta_{p}(\rho)\right) \subseteq\left[\frac{1}{4}, \infty\right)$, see remark 8.2. Since the metrics $g_{1}$ and $h_{1}$, implicit in the definition of $\Delta_{p}^{1}(\rho)$ as in Definition 10.1, are compact perturbations of $g$ and $h$, the underlying Hilbert spaces of $L^{2}$-sections can be identified by a bounded map $I$ which has bounded inverse and which moreover commutes with the operator $e^{-t T_{p, u}^{\rho}}$. In this way we can fix the Hilbert space. In particular, $e^{-t \Delta_{p}^{1}(\rho)}-e^{-t \Delta_{p}(\rho)}$ is trace class by Proposition 10.7, and thus it follows from the invariance of the essential spectrum under compact perturbations that $\Delta_{p}^{1}(\rho)$ has pure point spectrum in $[0,1 / 4)$. Moreover, it follows from [Mü5, Lemma 2.2] that there exist constants $c_{1}, C_{1}>0$ such that

$$
\operatorname{Tr}\left(e^{-t \Delta_{p}^{1}(\rho)}-e^{-t \Delta_{p}(\rho)}\right)-\operatorname{dim} \operatorname{Ker}\left(\Delta_{p}^{1}(\rho)\right) \leq C_{1} e^{-c_{1} t}
$$

for $t \geq 1$. Since

$$
\begin{aligned}
& \operatorname{Tr}_{\mathrm{rel}, u}\left(e^{-t \Delta_{p}^{1}(\rho)}\right)-\operatorname{dim} \operatorname{Ker}\left(\Delta_{p}^{1}(\rho)\right) \\
= & \operatorname{Tr}\left(e^{-t \Delta_{p}^{1}(\rho)}-e^{-t \Delta_{p}(\rho)}\right)-\operatorname{dim} \operatorname{Ker}\left(\Delta_{p}^{1}(\rho)\right)+\operatorname{Tr}_{\mathrm{rel}, u}\left(e^{-t \Delta_{p}(\rho)}\right),
\end{aligned}
$$

also the second estimate (10.8) follows.

Let $\Delta_{p}^{1}(\rho)$ be a compact perturbation of $\Delta_{p}(\rho)$. Then, modeling the corresponding definition on a compact manifold, we can define the relative zeta function of $\Delta_{p}(\rho)$ by

$$
\zeta_{\mathrm{rel}, u}\left(\Delta_{p}^{1}(\rho) ; s\right):=\frac{1}{\Gamma(s)} \int_{0}^{\infty} t^{s-1}\left(\operatorname{Tr}_{\mathrm{rel}, u} \Delta_{p}^{1}(\rho)-\operatorname{dim} \operatorname{Ker}\left(\Delta_{p}^{1}(\rho)\right)\right) d s .
$$

By the preceding two propositions, the integral is absolutely convergent for $\operatorname{Re}(s)>d / 2$ and using standard arguments, see for example Gi], these propositions also imply that 
it admits a meromorphic continuation to $\mathbb{C}$ which is reguar at $s=0$. In the same way, we can define the regularized zeta function $\zeta_{\text {reg }}\left(\Delta_{p}(\rho) ; s\right)$ using the regularized trace, see [MP2, Definition 7.13]. Thus, if $g_{1}$ and $h_{1}$ are compact perturbations of the metric $g$ on $X$ and $h$ on $E_{\rho}$ and if $\Delta_{p}^{1}(\rho)$ are the corresponding flat Hodge-Laplacians on $E_{\rho}$, one can define the relative analytic torsion generalizing the originial definition of Ray and Singer [RS] for compact manifolds:

$$
\log T_{\mathrm{rel}, u}\left(X, g_{1}, h_{1} ; E_{\rho}\right):=\left.\frac{1}{2} \sum_{p}(-1)^{p} p \frac{d}{d s}\right|_{s=0} \zeta_{\mathrm{rel}, u}\left(\Delta_{p}^{1}(\rho) ; s\right),
$$

where we have put $g_{1}$ and $h_{1}$ in the notation in order to indicate that, a priori, this torsion depends on these metrics. It will be shown in Proposition 15.1 that this is not the case for our present strongly acylic case $\rho \neq \rho_{\theta}$.

We finally remark that one can of course also define the regularized heat trace and the regularized analytic torsion for any compact perturbation $\Delta_{p}^{1}(\rho)$ of the flat Hodge Laplacian $\Delta_{p}(\rho), \rho \neq \rho_{\theta}$, in exactly the same way as one defines it for $\Delta_{p}(\rho)$. More precisely, by Proposition 10.7 the difference of the fibre traces $\operatorname{Tr} K_{\Delta_{p}^{1}(\rho)}(t, x, x)-\operatorname{Tr} K_{\Delta_{p}(\rho)}(t, x, x)$ is integrable over $X$ and thus by (10.1) the integral of $\operatorname{Tr} K_{\Delta_{p}^{1}(\rho)}(t, x, x)$ over $X(Y)$ has again an asymptotic expansion in $Y$ whose constant term one defines to be the regularized trace $\operatorname{Tr}_{\text {reg }}\left(e^{-t \Delta_{p}^{1}(\rho)}\right)$. Moreover, the above constructions immediately show that the difference $\operatorname{Tr}_{\mathrm{rel}, u}\left(e^{-t \Delta_{p}^{1}(\rho)}\right)-\operatorname{Tr}_{\mathrm{reg}}\left(e^{-t \Delta_{p}^{1}(\rho)}\right)$ equals the difference $\operatorname{Tr}_{\mathrm{rel}, u}\left(e^{-t \Delta_{p}(\rho)}\right)-\operatorname{Tr}_{\mathrm{reg}}\left(e^{-t \Delta_{p}(\rho)}\right)$ and by Proposition 10.8 this difference, and therefore also the difference of the two torsions, is a completely explicit function of the auxilliary parameter $u$. In summary, one can always pass back and forth between the regularized and relative objects.

\section{Some PROPERTIES OF THE HEAT KERNELS ON LOCALLY HOMOGENEOUS VECTOR BUNDLES}

In this section we firstly prove the trace class property for compactly supported differential operators applied to the heat kernel in order to treat the dependence of the torsion on compact perturbations of the metric later. Then we prove that for a fixed compact subset of $X$ the heat kernels we work with can be approximated in all derivatives by a sequence of heat kernels on closed manifolds following and argument of Lück, Schick and Bunke.

We let $X$ be the same manifold as in section 2.7 and we let $E_{\nu}$ be a locally homogeneous vector bundle over $X$. We let $D$ be a differential operator of order $\leq 2$ which acts on smooth sections of $E_{\nu}$ and which has compact support. By this we mean that there exists a compact set $\mathcal{V}$ such that $D \phi=0$ for all smooth sections $\phi$ of $E_{\nu}$ which vanish on $\mathcal{V}$. Then we have the following proposition.

Proposition 11.1. Let $B_{\nu}$ be a differential operator acting on the smooth sections of $E_{\nu}$ which is locally homogeneous at infinity. Then the operators $D \circ e^{-t B_{\nu}}$ and $e^{-t B_{\nu}} \circ D$ are trace class and their trace norm is uniformly bounded for $t$ in compact subsets of $(0, \infty)$.

Proof. Let $r$ be the function on $X$ defined in the previous section. We remark that for $C_{2}>0, T>0$ there exists a constant $C>0$ such that for all $x, y \in X$ and all $t \in(0, T]$ 
one can estimate

$$
r(x)^{n} r(y)^{n} e^{-C_{2} \frac{d^{2}(x, y)}{t}} \leq C r(x)^{3 n} e^{-C_{2} \frac{d^{2}(x, y)}{2 t}} .
$$

If $r(y) \leq r(x)^{2}$, this is clear and if $r(y)>r(x)^{2}$, then $d(x, y) \geq \frac{\log r(y)}{2}$ by the definition of the hyperbolic metric and the estimate also follows.

Now let $M_{r^{3 n}}, M_{r^{-3 n}}$ be the operators which arise by multiplication with $r^{3 n}$ resp. $r^{-3 n}$. Then we have

$$
D \circ e^{-t B_{\nu}}=\left(D \circ e^{-\frac{t}{2} B_{\nu}} \circ M_{r^{3 n}}\right) \circ\left(M_{r^{-3 n}} \circ e^{-\frac{t}{2} B_{\nu}}\right) .
$$

It suffices to show that each of the operators in brackets is Hilbert-Schmidt with HilbertSchmidt norm bounded on compact subsets of $(0, \infty)$. Let $I_{1}:=D \circ e^{-\frac{t}{2} B_{\nu}} \circ M_{r^{3 n}}$. Then $I_{1}$ is an integral operator with smooth kernel $K_{1}(t, x, y):=D_{x} K_{B_{\nu}}(t / 2, x, y) r^{3 n}(y)$, where $D_{x}$ indicates that we apply $D$ to the $x$-variable. Let $\mathcal{V}$ be the support of $D$. Since $B_{\nu}$ is elliptic and of second order, by the local Gårding inequality Gi there exists a constant $C>0$ such that

$$
\int_{X}\|D f(x)\|^{2} d x=\int_{\mathcal{V}}\|D f(x)\|^{2} \leq C\left(\int_{\mathcal{V}}\|f(x)\|^{2} d x+\int_{\mathcal{V}}\left\|B_{\nu} f(x)\right\|^{2} d x\right)
$$

for each smooth section $f$ of $E_{\nu}$. Therefore we get

$$
\begin{aligned}
& \left\|I_{1}\right\|_{H S}^{2}=\int_{X \times X}\left\|K_{1}(t, x, y)\right\|^{2} d x d y \leq C \int_{\mathcal{V} \times X}\left\|K_{B_{\nu}}(t / 2, x, y)\right\|^{2} r^{6 n}(y) d x d y \\
+ & C \int_{\mathcal{V} \times X}\left\|\left(B_{\nu}\right)_{x} K_{B_{\nu}}(t / 2, x, y)\right\|^{2} r^{6 n}(y) d x d y,
\end{aligned}
$$

where $\left(B_{\nu}\right)_{x}$ indicates that we apply $B_{\nu}$ to the $x$-variable. There exists a compact set $\tilde{\mathcal{V}}$ of $X$ such that for all $x \in \mathcal{V}$ and all $y \in X-\tilde{\mathcal{V}}$ one can estimate $d(x, y) \geq \frac{\log r(y)}{2}$. Therefore Proposition 10.3immediately implies that for $q \in\{0,1\}$ the norm $\left\|\left(B_{\nu}^{q}\right)_{x} K_{B_{\nu}}(t, x, y)\right\|^{2} r^{6 n}(y)$ is bounded on $\mathcal{V} \times X$ uniformly for $t$ in compact subsets of $(0, \infty)$ and since $X$ is of finite volume, $\left\|I_{1}\right\|_{H S}^{2}$ is finite and locally uniformly bounded in $t$.

Next, the operator $I_{2}:=M_{r-3 n} \circ e^{-\frac{t}{2} B_{\nu}}$ is an integral-operator with smooth kernel $K_{2}(t, x, y):=r^{-3 n}(x) K_{B_{\nu}}(t / 2, x, y)$ and by Proposition 10.3 and (11.1) this kernel is bounded on $X$ locally unifomrly in $t$. Since $X$ has finite volume, the same therefore holds for the Hilbert Schmidt norm of $I_{2}$. This proves the proposition for $D \circ e^{-t B_{\nu}}$. Since $e^{-t B_{\nu}} \circ D$ is the adjoint of $D^{*} \circ e^{-t B_{\nu}}$, the proposition follows also for $e^{-t B_{\nu}} \circ D$.

We let $X$ be the same manifold as in section 2.7 equipped with a metric $g_{1}$ which is a compact perturbation of the hyperbolic metric. Let $Y_{0}$ be as above. Then for $Y>Y_{0}$ we let $M(Y)$ be the closed orieted manifold which is the double of $X(Y+1)$ :

$$
M(Y):=X(Y+1) \sqcup_{\partial X(Y+1)}-X(Y+1),
$$

where $-X(Y+1)$ denotes the manifold $X(Y+1)$ with the reversed orienation. Taking a metric on the manifold with boundary $X(Y+1)$, which coincides with the metric $g_{1}$ in a neighbourhood of $X(Y)$ in $X(Y+1)$ and which is of product structure in a neighbourhood of the boundary $\partial X(Y+1)$, we can equipp $M(Y)$ with a metric which coincides with the 
hyperbolic metric in a neighbourhood of $X(Y) \subset M(Y)$ and of $-X(Y)$ in $M(Y)$. We let $E$ be a smooth Hermitian vector bundle over $X$ and we let $\Delta[X]$ be a differential operator which acts on the smooth sections of $E$ and which is of Laplace-type. We fix a smooth Hermitian vector bundle $E^{\prime}$ over $M(Y)$ such that the restrictions of $E$ and $E^{\prime}$ to $X(Y)$ are isometric. We shall from now on identify these bundles over $X(Y)$. If $E$ is flat, i.e. a bundle associated to a finite-dimensional representation of the fundamental group of $X(Y)$, then also $E^{\prime}$ can be chosen to be flat by Van Kampen's theorem. We fix an operator $\Delta[M(Y)]$ of Laplace type which acts on the smooth sections of $E^{\prime}$ and which has the property that it coincides with $\Delta[X]$ on smooth sections which are supported in the interior of $X(Y)$.

The operators $\Delta[M(Y)]$ and $\Delta[X]$ with domain the smooth (compactly supported) sections of the corresponding bundles are essentially selfadjoint and their closes will be denoted by the same symbol. We let $e^{-t \Delta[X]}$ and $e^{-t \Delta[M(Y)]}$ be their heat semigroups on the spaces of square integrable sections which act as integral operators with smooth kernels

$$
K_{\Delta[M(Y)]}(t, x, y) \in C^{\infty}\left(M(Y) \times M(Y), E^{\prime} \otimes\left(E^{\prime}\right)^{*}\right) ; K_{\Delta[X]}(t, x, y) \in C^{\infty}\left(X \times X, E \otimes E^{*}\right) .
$$

In the next proposition we show that for a fixed $Y_{1}>Y_{0}$ all derivatives of $K_{\Delta[X]}(t, x, y)$ can be approximated uniformly on $X\left(Y_{1}\right) \times X\left(Y_{1}\right)$ by the corresponding derivatives of the kernels $K_{\Delta[M(Y)]}(t, x, y)$ as $Y \rightarrow \infty$. We use an argument of Lück and Schick, [LS, Theorem 2.26], which they attribute to Bunke.

Proposition 11.2. Let $D$ be a differential operator which acts on the smooth sections of $E$. Let $T>0, Y_{1}>Y\left(\Gamma_{0}\right)$. Then there exist constants $C, c>0$ which depend only on $D$, $T, Y_{1}$ and $X$ such that for all $t \in(0, T)$, all $Y>Y_{1}$ and all $x_{0}, y_{0} \in X\left(Y_{1}\right)$ one has

$$
\left\|\frac{d^{i}}{d t^{i}} D_{x} K_{\Delta[X]}\left(t, x_{0}, y_{0}\right)-\frac{d^{i}}{d t^{i}} D_{x} K_{\Delta[M(Y)]}\left(t, x_{0}, y_{0}\right)\right\| \leq C e^{-\frac{c \operatorname{dist}^{2}\left(X\left(Y_{1}\right), \partial X(Y)\right)}{t}},
$$

where $i \in \mathbb{N}$ and where $D_{x}$ indicates that we apply $D$ to the first variable.

Proof. Let $\Delta:=\Delta[M(Y)]$ or $\Delta=\Delta[X]$. By the local Sobolev embedding theorem and by the local Gårding inequality [Gi], for $j \in \mathbb{N}$ there exists a constant $C_{j}\left(Y_{1}\right)$ which depends only on $j$ and $Y_{1}$ such that for all differential operators $D$ of order $\mu \in \mathbb{N}^{0}$ as in the proposition, for all $j \in \mathbb{N}$ with $j>d / 4+\mu / 2$, for all smooth sections $u$ of $E$ with compact support in $X\left(Y_{1}\right)$ and for all $x \in X\left(Y_{1}\right)$ one has

$$
\|D u(x)\| \leq C(D) C_{j}\left(Y_{1}\right)\left(\|u\|_{L^{2}\left(X\left(Y_{1}\right) ; E\right)}+\left\|\Delta^{j} u\right\|_{L^{2}\left(X\left(Y_{1}\right) ; E\right)}\right),
$$

where $C(D)$ is a constant that depends only on $D$. This implies in particular that it suffices to prove the proposition for $D=\Delta^{k}, k \in \mathbb{N}$. Then one can assume that $i=0$. We can now argue exactly as Lück and Schick in the proof of [LS, Theorem 2.26]. We assume that $Y \geq Y_{1}+2$. Let $u$ be a smooth sections with compact support in the interior of $X\left(Y_{1}\right)$. Then as in [LS], we consider the section

$$
f:=\left(\Delta[M(Y)]^{k} e^{-t \Delta[M(Y)]}-\Delta[X]^{k} e^{-t \Delta[X]}\right) u,
$$


which is defined on $X(Y)$, in particular on $X\left(Y_{1}\right)$. Let $m, l \in \mathbb{N}$. As in [LS], if $\Delta=\Delta[X]$ or $\Delta=\Delta[M(Y)]$ the spectral theorem implies that restricted to $L^{2}\left(X\left(Y_{1}\right) ; E\right)$ one has

$$
\begin{aligned}
& \Delta^{m} \Delta^{l} f \\
= & \int_{0}^{\infty} t^{-2(m+l+k)-\frac{1}{2}} P_{m, l, k}(s, \sqrt{t}) e^{-\frac{s^{2}}{4 t}}(\cos (s \sqrt{\Delta[M(Y)]})-\cos (s \sqrt{\Delta[X]})) u d s,
\end{aligned}
$$

where $P_{m, l, k}$ is a universal polynomial depending only on $m, l$ and $k$. By unit propagation speed of the wave equation [Ta], for $s \in(0, \infty)$ one has $\operatorname{supp} \cos (s \sqrt{\Delta[X]}) u \subset\{x \in$ $X: \operatorname{dist}(x, \operatorname{supp} u) \leq s\}$ and $\operatorname{supp} \cos (s \sqrt{\Delta[M(Y)]}) u \subset\{x \in M(Y): \operatorname{dist}(x, \operatorname{supp} u) \leq s\}$. Using the uniqueness of solutions of the wave equation with inital datum $u$, it follows that

$$
\cos (s \sqrt{\Delta[X]}) u=\cos (s \sqrt{\Delta[M(Y)]}) u ; \quad \text { if } s \leq \operatorname{dist}\left(X\left(Y_{1}\right), \partial X(Y)\right) .
$$

Since $\cos s \Delta$ is of norm $\leq 1$, (11.4) and (11.5) imply that

$$
\left\|\Delta^{m} \Delta^{l} f\right\|_{L^{2}\left(X\left(Y_{1}\right) ; E\right)} \leq C_{k, l, m} e^{-c_{k, l, m} \frac{\operatorname{dist}\left(X\left(Y_{1}\right), \partial X(Y)\right)^{2}}{t}}\|u\|_{L^{2}\left(X\left(Y_{1}\right) ; E\right)},
$$

where $c_{k, l, m}$ and $C_{k, l, m}$ are universal constant that depend only on $k, l, m$. If we apply (11.3) choosing $m$ appropriately, it follows that there exists a constant $C_{k, l}\left(Y_{1}\right)$ which depends only on $k, l$ and $Y_{1}$ and a constant $c_{k, l}$, which depends only on $k$ and $l$, such that for $x \in X\left(Y_{1}\right)$ one has

$$
\left\|\Delta^{l} f(x)\right\| \leq C_{k, l}\left(Y_{1}\right) e^{-c_{k, l} \frac{\operatorname{dist}\left(X\left(Y_{1}\right), \partial X(Y)\right)^{2}}{t}}\|u\|_{L^{2}\left(X\left(Y_{1}\right) ; E\right)}
$$

In other words, for $x \in X\left(Y_{1}\right)$ one has

$$
\begin{aligned}
& \left\|\int_{X\left(Y_{1}\right)}\left(\Delta_{x}^{l} \Delta_{x}^{k} K_{\Delta[M(Y)]}(t, x, y)-\Delta_{x}^{l} \Delta_{x}^{k} K_{\Delta[X]}(t, x, y)\right) u(y) d y\right\| \\
& =\left\|\int_{X\left(Y_{1}\right)}\left(\Delta_{y}^{l} \Delta_{x}^{k} K_{\Delta[M(Y)]}(t, x, y)-\Delta_{y}^{l} \Delta_{x}^{k} K_{\Delta[X]}(t, x, y)\right) u(y) d y\right\| \\
& \leq C_{k, l}\left(Y_{1}\right) e^{-c_{k, l} \frac{\operatorname{dist}\left(X\left(Y_{1}\right), \partial X(Y)\right)^{2}}{t}}\|u\|_{L^{2}\left(X\left(Y_{1}\right) ; E\right)} .
\end{aligned}
$$

Since the smooth sections $u$ with compact interior support are dense in $L^{2}\left(X\left(Y_{1}\right) ; E\right)$, it follows that for each $x \in X\left(Y_{1}\right)$ one has

$$
\left\|\Delta_{y}^{l} \Delta_{x}^{k} K_{\Delta[M(Y)]}(t, x,-)-\Delta_{y}^{l} \Delta_{x}^{k} K_{\Delta[X]}(t, x,-)\right\|_{L^{2}\left(X\left(Y_{1}\right) ; E\right)} \leq C_{k, l}\left(Y_{1}\right) e^{-c_{k, l} \frac{\operatorname{dist}\left(X\left(Y_{1}\right), \partial X(Y)\right)^{2}}{t}} .
$$

Choosing $l$ appropriately and applying (11.3) again, it follows that there exists a constant $C_{k}\left(Y_{1}\right)$ which depends only on $k$ and $Y_{1}$ and a constant $c_{k}$ which depens only on $k$ such that all $x, y \in X\left(Y_{1}\right)$ one has

$$
\left\|\Delta_{x}^{k} K_{\Delta[M(Y)]}(t, x, y)-\Delta_{x}^{k} K_{\Delta[X]}(t, x, y)\right\| \leq C_{k}\left(Y_{1}\right) e^{-c_{k} \frac{\operatorname{dist}\left(X\left(Y_{1}\right), \partial X(Y)\right)^{2}}{t}}
$$

and the proposition is proved. 


\section{The Variation of the De Rham Complex After Vishik and Lesch}

In this section we introduce the variation of the De Rham complex, due to Vishik and Lesch, fort the present case.

To keep this article reasonably self-contained, let us briefly recall some of those concepts and results of Brüning and Lesch [BL] which we shall use in this article. Firstly, a Hilbert complex is a collection of Hilbert spaces $H_{i}, i=0, \ldots, n, n \in \mathbb{N}$ together with closed (in general unbounded) operators $D_{i}$ with domain $\operatorname{dom}\left(D_{i}\right) \subseteq H_{i}$ and with $D_{i}: \operatorname{dom}\left(D_{i}\right) \rightarrow$ $H_{i+1}$ such that $\mathcal{R}_{i}:=D_{i}\left(\operatorname{dom}\left(D_{i}\right)\right) \subset \operatorname{dom}\left(D_{i+1}\right)$ and such that $D_{i+1} \circ D_{i}=0$. We assume that each $D_{i}$ is densely defined. Let $D_{i}^{*}$ be the adjoint of $D_{i}$. Then the $i$-th Laplacian $\Delta_{i}$ of the Hilbert complex is defined as

$$
\Delta_{i}:=D_{i}^{*} \circ D_{i}+D_{i-1} \circ D_{i-1}^{*},
$$

where we use the usual conventions for the domains of sums and compositions of unbounded operators. Using the Gauss-Bonnet operator of the complex [BL] and von Neumann's theorem, one can show that each operator $\Delta_{i}$ is selfadjoint. We denote by $\mathcal{H}_{i}:=\operatorname{ker}\left(D_{i}\right) / \mathcal{R}_{i-1}$ the cohomology of the Hilbert complex. A Hilbert complex is called a Fredholm complex if $\mathcal{R}_{i}$ is closed and if $\mathcal{H}_{i}$ is finite-dimensional. Let $\hat{\mathcal{H}}_{i}:=\operatorname{ker}\left(\Delta_{i}\right)$. One has $\hat{\mathcal{H}}_{i}=$ $\operatorname{Ker}\left(D_{i}\right) \cap \operatorname{ker}\left(D_{i-1}^{*}\right)$ and thus one has a canonical map $\hat{\mathcal{H}}_{i} \rightarrow \mathcal{H}_{i}$. In all the situations that occur in the present article, this map will be an isomorphism by the strong Hodge decomposition theorem for Fredholm complexes due to Brüning and Lesch:

Proposition 12.1. A Hilbert complex is a Fredholm complex if and only if 0 does not belong to the essential spectrum of $\Delta_{i}$ for each $i$. For a Fredholm complex the canonical map from $\hat{\mathcal{H}}_{i}$ to $\mathcal{H}_{i}$ is an isomorphism for each $i$.

Proof. [BL, Theorem 2.4, Corollary 2.5] .

The Hilbert complexes we work with will arise in the following context; we of course refer again to [BL] for a much broader treatment. We let $M$ be a smooth Riemannian manifold with metric $g$ and with smooth boundary $\partial M$, which might be empty. We let $F$ be a flat Hermitian vector bundle over $M$ with Hermitian fibre metric $h$, which we do not necessarily assume to be flat. We let $\Lambda^{p} F:=\Lambda^{p} T^{*} M \otimes F$. The underlying Hilbert spaces are the spaces $L^{2}\left(M ; \Lambda^{p} F\right)$ of square integrable section of $\Lambda^{p} F$. If $M$ is not compact, then these spaces depend on the metrics $g$ and $h$. Let $\Omega_{c}^{p}(M ; F)$ be the set of smooth $F$-valued $p$-forms on $M$ with compact support in the interior of $M$ and let $d_{p}: \Omega_{c}^{p}(M ; F) \rightarrow \Omega_{c}^{p+1}(M ; F)$ be the exterior derivative. Then one obtains a complex $\left(\Omega_{c}^{*}(M: F), d\right)$. In order to obtain a Hilbert complex, one needs to specify suitable closed extensions of the $d_{p}$. Thus for each $p$ let $d_{p}^{t}$ be the transposed differential operator of $d_{p}$, i.e. $d_{p}^{t}: \Omega_{c}^{p+1}(M ; F) \rightarrow \Omega_{c}^{p}(M ; F)$ is the unique first order differential operator which satisfies $\left\langle d_{p}^{t} \phi, \psi\right\rangle_{L^{2}\left(M ; \Lambda^{p} F\right)}=\left\langle\phi, d_{p} \psi\right\rangle_{L^{2}\left(M ; \Lambda^{p+1} F\right)}$ for all $\phi \in \Omega_{c}^{p+1}(M ; F)$ and all $\psi \in \Omega_{c}^{p}(M ; F)$. Then one defines the maximal extension of $d_{p}$ by $d_{p ; \max }:=\left(d_{p}^{t}\right)^{*}$, which is closed. Then $\operatorname{dom}\left(d_{P ; \max }\right)$ is set of all $\omega \in L^{2}\left(M ; \Lambda^{p} F\right)$ for which $d \omega \in L^{2}\left(M ; \Lambda^{p+1} F\right)$, where $d \omega$ is meant in the distributional sense. One defines the minimal extension of $d_{p}$ as its closure: 
$d_{p ; \min }:=\overline{d_{p}}$. We let $\Omega_{\max / \min }^{p}(M ; F):=\operatorname{dom}\left(d_{p ; \max / \min }\right)$. Then by [BL, Lemma 3.1] these spaces together with the operators $d_{p ; \max / \min }$ define Hilbert complexes $\mathcal{D}_{\max / \min }^{*}(M ; F)$. Any Hilbert complex lying between $\mathcal{D}_{\text {min }}^{*}(M ; F)$ and $\mathcal{D}_{\text {max }}^{*}(M ; F)$ is called an ideal boundary condition.

Now we let $X$ be the same manifold as in section 2.7. We let $Y_{0}$ be as in that section and we let $Y_{1}>Y_{0}+2$. We have an inclusion $X\left(Y_{1}\right) \subset X$ and we fix a metric $g_{1}$ on $X$ which is of product structure on a neighourhood of $X\left(Y_{1}+1 / 2\right) \backslash X\left(Y_{1}-1 / 2\right)$ in $X$ and which coincides with the hyperbolic metric $g$ of $X$ on the set $X\left(Y_{1}-1\right)$ and on the set $X \backslash X\left(Y_{1}+1\right)$. Thus $g_{1}$ is a compact perturbation of $g$ which is of product structure on some fixed cusp pieces. To save notation, we write

$$
X^{+}:=X\left(Y_{1}\right) ; \quad X^{-}:=X \backslash X^{+}=\bigsqcup_{P \in \mathfrak{P}} F_{P}\left(Y_{1}\right) ; \quad Z:=\partial X^{+} .
$$

We equip $X^{ \pm}$with the metric $g_{1}$. We let $\rho \in \operatorname{Rep}(G)$ such that $\rho \neq \rho_{\theta}$. For brevity, we let $F:=E_{\rho}$ denote the corresponding flat vector bundle over $X$, where $E_{\rho}$ is as in section 3. We let $h$ be the metric on $F$ introduced in section 3. Then we let $h_{1}$ be a metric on $F$ which is of product structure in a neighbourhood of $X\left(Y_{1}+1 / 2\right)-X\left(Y_{1}-1 / 2\right)$ and which coincides with the metric $h$ on the set $X\left(Y_{1}-1\right)$ and on the set $X \backslash X\left(Y_{1}+1\right)$. Now we consider the collar $W:=(-1 / 2,1 / 2) \times Z$ which we embed isometrically into $\left(X, g_{1}\right)$ such that $(-1 / 2,0) \times Z$ is isometric to a neighbourhood of $Z$ in $X^{-}$and such that $(0,1 / 2) \times Z$ is isometric to a neighbourhood of $Z$ in $X^{+}$. We let $\phi \in C_{c}^{\infty}((-1 / 2,1 / 2) \times Z)$ be a smooh $\mathbb{R}$-valued function such that $\phi \equiv 1$ in a neighbourhood of $\{0\} \times Z$ in $(-1 / 2,1 / 2) \times Z$ and such that $\phi(-t, p)=\phi(t, p)$ for all $(t, p) \in(-1 / 2,1 / 2) \times Z$. Then we consider the manifolds with two components:

$$
W^{c u t}:=(-1 / 2,0] \times Z \bigsqcup[0,1 / 2) \times Z ; \quad X^{c u t}:=X^{-} \bigsqcup X^{+} .
$$

The manifold $X$ is complete and therefore by $\mathrm{Ch}$ the corresponding Laplacians with domains the smooth compactly supported sections are essentially selfadjoint. Thus by [BL, Lemma 3.3] the De Rham complex of $F$-valued differential forms on $X$ has a unique ideal boundary condition $\mathcal{D}^{*}(X ; F)$. We will denote the restriction of $F$ to $X^{ \pm}$by the same letter. Then we use the notation

$$
\mathcal{D}^{*}\left(X^{ \pm} ; F\right):=\mathcal{D}_{\text {max }}^{*}\left(X^{ \pm} ; F\right), \quad \mathcal{D}^{*}\left(X^{ \pm}, Z ; F\right):=\mathcal{D}_{\text {min }}^{*}\left(X^{ \pm} ; F\right) .
$$

We point out that in this section we equip $X, X^{ \pm}$and $F$ with compact perturbations of the original metrics; thus the flat Hodge Laplacians are only compact deformations of the original flat Hodge Laplacians in the sense of section 10. Since $X^{+}$is a compact manifold with boundary, the cohomology of $\mathcal{D}^{*}\left(X^{+} ; F\right)$ equals the De Rham or singular cohomology of $X^{+}$with values in $F$, see for example [BL, Theorem 4.1], where the proof extends without difficulty to the flat bundle $F$. Therefore, we shall just denote it unambiguously by $H^{*}\left(X^{+} ; F\right)$. On the other hand, we will denote the cohomology of the complex $\mathcal{D}^{*}\left(X^{-}, Z ; F\right)$ by $H_{\min }^{*}\left(X^{-}, Z ; F\right)$ in order to distinguish it from the corresponding De Rham cohmology - since $X^{-}$retracts onto $Z$, this De Rham cohomology is of course 
always trivial. This is not the case for the cohomology $H_{\min }^{*}\left(X^{-}, Z ; F\right)$. More precisely, as in [Le, section 4.2], one can consider the long exact sequence of Hilbert complexes

$$
0 \longrightarrow \mathcal{D}^{*}\left(X^{-}, Z ; F\right) \stackrel{\alpha}{\longrightarrow} \mathcal{D}^{*}(X ; F) \stackrel{\beta}{\longrightarrow} \mathcal{D}^{*}\left(X^{+} ; F\right) \longrightarrow 0,
$$

where $\alpha$ is extension by zero and $\beta$ is restriction to $X^{+}$. To prove the exactness of this sequence, we firstly remark that it follows immediately from the definitions that $\alpha$ and $\beta$ are indeed maps between the involved Hilbert complexes. If one multiplies with suitable cutoff functions, it follows easily that the exactness of (12.2) is a purely local question in a neighbourhood of the boundary $Z$. Therefore, it suffices to prove the exactness if one replaces $X$ by the double $X^{+} \sqcup_{Z}-X^{+}$of $X^{+}$and $X^{-}$by $-X^{+}$, i.e. $X^{+}$with reversed orientation. For the situation of the double, the exactness follows from [BL, equation (4.12)]: If $\alpha^{*}$ is the corresponding involution introduced on page 121 in [BL], and if a form $\omega \in \mathcal{D}^{*}\left(X^{+} \sqcup_{Z}-X^{+} ; F\right)$ satisfies $\omega \mid X^{+}=0$, then $\left.\omega\right|_{-X^{+}}=\left.\left(\omega-\alpha^{*} \omega\right)\right|_{-X^{+}}$and therefore $\omega$ is in the image of the map $\mathcal{D}^{*}\left(-X^{+}, Z ; F\right) \rightarrow \mathcal{D}^{*}\left(-X^{+} \sqcup_{Z} X^{+} ; F\right)$. This proves exactness in the midde. The surjectivitiy of $\beta$ immediately follows from [BL, equation (4.12)]. The exact sequence (12.2) induces a long exact cohomology sequence. Let

$$
\delta_{p}: H^{p}\left(X^{+} ; F\right) \rightarrow H_{\min }^{p+1}\left(X^{-}, Z ; F\right)
$$

be the $p$-th connecting homomorphism of that sequence. Then we have the following Proposition (let us recall that $F$ was the flat vector bundle associated to a representation $\rho$ which satisfies $\left.\rho \neq \rho_{\theta}\right)$ :

Proposition 12.2. The cohomology of the complex $\mathcal{D}^{*}(X ; F)$ vanishes. In particular for each $p$ the map $\delta_{p}$ is an isomorphism and the cohomology $H_{\min }^{*}\left(X^{-}, Z ; F\right)$ is finitedimensional. Moreover, $\mathcal{D}^{*}\left(X^{-}, Z ; F\right)$ is a Fredholm complex.

Proof. By [BL, Theorem 3.5], the cohomology of $\mathcal{D}^{*}(X ; F)$ equals the corresponding $L^{2}$ cohomology. This cohomology is zero by Proposition 8.1 . We recall that $g_{1}$ and $h_{1}$ are compact perturbations of the metrics $g$ and $h$ used in that proposition and hence belong to the quasi isometry class of $g$ and $h$. The last statement follows from [BL, Theorem 2.4 $(2)]$.

Next we use the variation of the De Rham complex $\mathcal{D}^{*}(X ; F)$ due to Vishik and Lesch. Let $\iota_{ \pm}: Z \hookrightarrow X^{ \pm}$denote the inclusions. For $\theta \in \mathbb{R}$ and each $p$ let

$$
\mathcal{D}_{\theta}^{p}(X ; F):=\left\{\left(\omega_{1}, \omega_{2}\right) \in \mathcal{D}^{p}\left(X^{-} ; F\right) \oplus \mathcal{D}^{p}\left(X^{+} ; F\right): \cos \theta \cdot \iota_{-}^{*} \omega_{1}=\sin \theta \cdot \iota_{+}^{*} \omega_{2}\right\},
$$

where, as in [Le, section 4.2], $\iota_{ \pm}^{*}(\omega)$ are boundary values defined by traces on Sobolev spaces. More precisely, by Paq, Theorem 1.9], $\iota_{ \pm}^{*}$ are well defined on $\mathcal{D}^{p}\left(X^{ \pm} ; F\right)$ and $\mathcal{D}^{p}\left(X^{ \pm}, Z ; F\right)$ coincides with the kernel of $\iota_{ \pm}^{*}$. Again, the non-compactness of $X^{-}$is not an issue here, since one can localize everything to a neighourhood of $Z$ and $Z$ is compact. The flat bundle isn't an issue either here. For each p let $d_{p}^{\theta}: \mathcal{D}_{\theta}^{p}(X ; F) \rightarrow \mathcal{D}_{\theta}^{p+1}(X ; F)$ be the restriction of the direct sum of the operators $d_{p ; \max }$ on $\mathcal{D}^{p}\left(X^{-} ; F\right)$ and on $\mathcal{D}^{p}\left(X^{+} ; F\right)$. Then the $\mathcal{D}_{\theta}^{p}(X ; F)$ with $d_{p}^{\theta}$ form a subcomplex $\mathcal{D}_{\theta}^{*}(X ; F)$ of $\mathcal{D}^{*}\left(X^{-} ; F\right) \oplus \mathcal{D}^{*}\left(X^{+} ; F\right)$. By 
the previous remark one has

$$
\mathcal{D}_{\theta=0}^{*}(X ; F)=\mathcal{D}^{*}\left(X^{-}, Z ; F\right) \oplus \mathcal{D}^{*}\left(X^{+} ; F\right),
$$

Furthermore, arguing in the same way as after (12.2), one can show that one has a natural isomorphism of complexes

$$
\mathcal{D}_{\theta=\frac{\pi}{4}}^{*}(X ; F) \cong \mathcal{D}^{*}(X ; F),
$$

[Vi, page 16], [Le, page 22]. Also as in (12.2) one has a short sequence

$$
0 \longrightarrow \mathcal{D}^{*}\left(X^{-}, Z ; F\right) \stackrel{\alpha_{\theta}}{\longrightarrow} \mathcal{D}_{\theta}^{*}(X ; F) \stackrel{\beta_{\theta}}{\longrightarrow} \mathcal{D}^{*}\left(X^{+} ; F\right) \longrightarrow 0
$$

where $\alpha_{\theta}\left(\omega_{1}\right):=\left(\omega_{1}, 0\right)$ and where $\beta_{\theta}\left(\omega_{1}, \omega_{2}\right):=\omega_{2}$.

The complexes $\mathcal{D}_{\theta}^{*}(X ; F)$ have varying domains; following Lesch, [Le, section 5], one can transform them into complexes with fixed domains. Thus let $S: W^{\text {cut }} \rightarrow W^{\text {cut }}$ be the reflection map, given by $S((t, p)):=(-t, p)$. Then define

$$
T: \Omega^{*}\left(W^{\text {cut }} ; F\right) \rightarrow \Omega^{*}\left(W^{c u t} ; F\right), \quad T\left(\omega_{1}, \omega_{2}\right):=\left(S^{*} \omega_{2},-S^{*} \omega_{1}\right) .
$$

One has

$$
\left(\cos \left(\theta+\theta^{\prime}\right), \quad-\sin \left(\theta+\theta^{\prime}\right)\right) \cdot\left(\begin{array}{cc}
\cos \theta & \sin \theta \\
-\sin \theta & \cos \theta
\end{array}\right)=\left(\begin{array}{ll}
\cos \theta^{\prime}, & -\sin \theta^{\prime}
\end{array}\right) .
$$

Thereore, if one define

$$
\Phi_{\theta}:=\cos (\theta \phi) \mathrm{Id}+\sin (\theta \phi) T: \Omega^{*}\left(W^{c u t} ; F\right) \rightarrow \Omega^{*}\left(W^{c u t} ; F\right),
$$

then $\Phi_{\theta}$ canonically extends to a unitary transformation of $L^{2}\left(X, \Lambda^{*} T^{*} X \otimes F\right)$ and applying (12.7) it follows that $\Phi_{\theta}$ maps $\mathcal{D}_{\theta^{\prime}}^{p}(X ; F)$ onto $\mathcal{D}_{\theta+\theta^{\prime}}^{p}(X ; F)$. Finally, since $T$ commutes with exterior differentiation, on $\mathcal{D}_{\theta^{\prime}}^{*}(X ; F)$ one has $\Phi_{\theta}^{*} d^{\theta+\theta^{\prime}} \Phi_{\theta}=d^{\theta^{\prime}}+\theta \operatorname{ext}(d \phi) T$, see [Le, Lemma 5.1]. Thus if one lets

$$
\tilde{d}_{p}^{\theta}:=d_{p}^{\frac{\pi}{4}}+\theta \operatorname{ext}(d \phi) T
$$

with domain $\mathcal{D}_{\theta=\pi / 4}^{p}(X ; F)$, one obtains a complex $\tilde{\mathcal{D}}_{\theta}^{*}(X ; F)$ with fixed domain which is isometric to the complex $\mathcal{D}_{\theta}^{*}(X ; F)$. Since $\theta$ ext $(d \phi) T$ is bounded and since one can identifty $d_{p}^{\pi / 4}$ with $d_{p}$ on $\mathcal{D}^{*}(X ; F)$, it follows that the complexes $\mathcal{D}_{\theta}^{*}(X ; F)$ are Hilbert complexes too. Let $\Delta_{p}^{\theta}$ resp. $\tilde{\Delta}_{p}^{\theta}$ be the Laplacians of $\mathcal{D}_{\theta}^{*}(X ; F)$ resp. $\tilde{\mathcal{D}}_{\theta}^{*}(X ; F)$. Let

$$
\begin{aligned}
\frac{d}{d \theta} \tilde{\Delta}_{p}^{\theta} & :=\left(\tilde{d}_{p}^{\theta}\right)^{*}(\operatorname{ext}(d \phi) T)+(\operatorname{ext}(d \phi) T)^{*} \tilde{d}_{p}^{\theta} \\
& +\left(\tilde{d}_{p-1}^{\theta}\right)(\operatorname{ext}(d \phi) T)^{*}+(\operatorname{ext}(d \phi) T)\left(\tilde{d}_{p-1}^{\theta}\right)^{*}
\end{aligned}
$$

Then $\frac{d}{d \theta} \tilde{\Delta}_{p}^{\theta}$ is a closed operator with domain containing $\operatorname{dom}\left(\Delta_{p}\right)$. Moreover, for each $\phi \in \operatorname{dom}\left(\Delta_{p}\right)$ one obviously has in $L^{2}$ :

$$
\lim _{\theta^{\prime} \rightarrow \theta} \frac{\left(\tilde{\Delta}_{p}^{\theta^{\prime}}-\tilde{\Delta}_{p}^{\theta}\right) \phi}{\theta^{\prime}-\theta}=\frac{d}{d \theta} \tilde{\Delta}_{p}^{\theta} \phi
$$


Therefore, the family $\tilde{\Delta}_{p}^{\theta}$ is a smooth one-parameter family of selfajoint operators with constant domains. We remark that the operators $\tilde{\Delta}_{p}^{\theta}$ are not differential operators since $T$ doesn't decrease supports, see [Le, page 24]. However, outside the collar where the variation takes place, they are differential operators.

\section{The HeAt KeRnel OF THE PERTURBED LAPLACIAN}

We keep the notation of the preceding section. By [Le, Lemma 3.1], the operator $e^{-t \tilde{\Delta}_{p}^{\theta}}$ acts as an integral operator with smooth kernel $K_{\tilde{\Delta}_{p}^{\theta}}$. For the purposes of the present paper, we will need to construct this kernel explicitly. We use the parametrix method to obtain the kernel by patching together the original heat Kernel of $\Delta_{p}$ with the kernel of the perturbed operaterator on the double of $X^{+}$. This method of patching togehter heat kernels can be found in [RS, page $184 \mathrm{ff}]$, [BGM, E III], [Do1, chapter 4], [Mü3, chapter VII], for example .

We continue to work with the metric $g_{1}$ on $X$ and the metric $h_{1}$ on $F:=E_{\rho}$. Consider the double $-X^{+} \sqcup_{\partial X^{+}} X^{+}$of $X^{+}$, where $-X^{+}$denotes $X^{+}$with the reversed orientation. Let us recall that $X^{+}$is a compact manifold with boundary and that near its boundary all structures are product. On this double, one can perform exactly the same variation as described above, replacing $X^{-}$by $-X^{+}$. We denote the corresponding bundle by $F$ too and we denote the corresponding operators by $\Delta_{p}^{\theta}, \tilde{\Delta}_{p}^{\theta}$ too. Then the integral kernel $E_{t}^{p, \theta}(x, y)$ of $e^{-t \Delta_{p}^{\theta}}$ acting on $L^{2}\left(-X^{+} \sqcup X^{+} ; F\right)$ can be computed explicitly [Le, equation 5.22]: Let $E_{t}^{p}$ denote the kernel of the $p$-th F-valued Hodge-Laplacian on the closed manifold $-X^{+} \sqcup_{\partial X^{+}} X^{+}$. Then one has

$$
E_{t}^{p, \theta}(x, y)= \begin{cases}E_{t}^{p}(x, y) \pm \cos (2 \theta)\left(S^{*} \circ E_{t}^{p}\right)(x, y) & \text { if } x, y \in \pm X^{+} \\ \sin (2 \theta) E_{t}^{p}(x, y) & \text { if } x \in \pm X^{+}, y \in \mp X^{+}\end{cases}
$$

where we have put $+X^{+}:=X^{+}$. Thus, if $\Phi_{\theta}$ is now defined on $L^{2}\left(-X^{+} \sqcup X^{+} ; F\right)$, the integral kernel $\tilde{E}_{t}^{p, \theta}$ of $e^{-t \tilde{\Delta}_{p}^{\theta}}$ is given by

$$
\tilde{E}_{t}^{p, \theta}=\Phi_{\frac{\pi}{4}-\theta} \circ E_{t}^{p, \theta} \circ \Phi_{\frac{\pi}{4}-\theta}^{-1} .
$$

Equations (12.8) and (13.1) immediately imply that $\tilde{E}_{t}^{p, \theta}(x, y)$ depends smoothly on $\theta$ and using the Gaussian bounds of $E_{t}^{p}$ on the closed manifold $-X^{+} \sqcup_{\partial X^{+}} X^{+}$(see for example $\mathrm{Gr}$, Theorem 1.4.3.1]), they imply that for each $T>0$ there exists constants $C_{1}, C_{2}>0$ such that for all $t \in(0, T]$ and all $x, y \in-X^{+} \sqcup_{\partial X^{+}} X^{+}$and $i \in\{0,1\}$ one has

$$
\left\|\left(\tilde{\Delta}_{p}^{\theta}\right)^{i} \tilde{E}_{t}^{p, \theta}(x, y)\right\|=\left\|\frac{d^{i}}{d t^{i}} \tilde{E}_{t}^{p, \theta}(x, y)\right\| \leq C_{1} t^{-\frac{d}{2}-i} e^{-C_{2} \frac{d^{2}(x, y)}{t}} .
$$

Now let $K_{\Delta_{p}}(t, x, y)$ be the integral kernel of the original $F$-valued Laplacian $\Delta_{p}$ on $X$, where we of course still use the metrics $g_{1}$ and $h_{1}$. This kernel satisfies the properties of Proposition 10.3. Let $\Phi_{1}$ and $\Psi_{1}$ be smooth functions on $X$ which have compact support in $X\left(Y_{1}+1 / 2\right)$, which are identically 1 on a neighbourhood of $X\left(Y_{1}\right) \cup \operatorname{supp}(\phi)$, where $\phi$ is as in the previous section, and which satisfy $\Phi_{1}=1$ on a neighbourhood of $\operatorname{supp}\left(\Psi_{1}\right)$. 
Let $\Psi_{2}:=1-\Psi_{1}$ and fix a smooth function $\Phi_{2}$ such that $\Phi_{2}=1$ on a neighbourhood of $\operatorname{supp}\left(\Psi_{2}\right)$ and such that $\Phi_{2}=0$ on a neighbourhood of $\operatorname{supp}(\phi)$. Then one defines

$$
P(t, x, y ; \theta):=\Phi_{1}(x) \tilde{E}_{t}^{p, \theta}(t, x, y) \Psi_{1}(y)+\Phi_{2}(x) K_{\Delta_{p}}(t, x, y) \Psi_{2}(y) .
$$

Let

$$
Q_{1}(t, x, y ; \theta):=\left(\frac{d}{d t}+\tilde{\Delta}_{p}^{\theta}\right) P(t, x, y ; \theta)
$$

where $\tilde{\Delta}_{p}^{\theta}$ is applied to the first variable. Since $\Phi_{1,2}=1$ on neighbourhoods of $\operatorname{supp}\left(\Psi_{1,2}\right)$ and since $\tilde{\Delta}_{p}^{\theta}$ equals the corresponding operator on $X^{+} \sqcup-X^{+}$on $\operatorname{supp}\left(\Phi_{1}\right)$ resp. equals $\Delta_{p}$ on $\operatorname{supp}\left(\Phi_{2}\right)$, there exists an $\epsilon>0$ such that

$$
Q_{1}(t, x, y ; \theta)=0, \quad \text { if } d(x, y)<\epsilon .
$$

Moreover, there exists a compact subset $\mathcal{V}$ of $X$ such that $Q_{1}(t, x, y ; \theta)=0$ if $x \notin \mathcal{V}$. Thus invoking Proposition 10.3, (13.3) and the local Gårding inequality on $\mathcal{V}$, it follows easily that for each $T>0$ and $l \in \mathbb{N}$ there exist constants $C_{1}$ and $C_{2}$ such that for all $t \in(0, T]$ one can estimate

$$
\left\|Q_{1}(t, x, y ; \theta)\right\| \leq t^{l} C_{1} r(y)^{n} e^{-C_{2} \frac{d^{2}(x, y)}{t}}
$$

for all $x, y \in X$, where we make from now on the convention that we use $C, C_{1}, C_{2}$ as constants in every estimate. In particular, $P$ is a parametrix or an approximate solution of the heat equation in the sense of [BGM] resp. [BGV]. Now define inductively

$$
Q_{k+1}(t, x, y ; \theta):=\int_{0}^{t} \int_{X} Q_{1}(t-s, x, w ; \theta) \circ Q_{k}(s, w, y ; \theta) d w d s .
$$

The inner integral ranges over the fixed compact set $\mathcal{V}$ only and applying (13.7) and the elementary inequality

$$
\frac{d^{2}(x, w)}{t-s}+\frac{d^{2}(w, y)}{s} \geq \frac{d^{2}(x, y)}{t}
$$

$0<s<t$, [Do1, page 67], one shows by induction in $k$ that

$$
\left\|Q_{k}(t, x, y ; \theta)\right\| \leq C_{1} r(y)^{n} t^{l} \frac{t^{k}}{k !} e^{-C_{2} \frac{d^{2}(x, y)}{t}} .
$$

In particular, the series

$$
Q(t, x, y ; \theta):=\sum_{k=1}^{\infty}(-1)^{k} Q_{k}(t, x, y ; \theta)
$$

converges absolutely and satisfies

$$
\|Q(t, x, y ; \theta)\| \leq C_{1} t^{l} r(y)^{n} e^{-C_{2} \frac{d^{2}(x, y)}{t}} .
$$

Let

$$
P * Q(t, x, y, \theta):=\int_{0}^{t} \int_{X} P(t-s, x, w ; \theta) \circ Q(s, w, y ; \theta) d w d s .
$$


This term can be estimated as follows. Since the inner integral in (13.12) ranges over the fixed compact set $\mathcal{V}$ only, the estimates from Proposition 10.3. (13.3) and (13.11) imply that

$$
\begin{aligned}
r(x)^{j} r(y)^{j}\|P * Q(t, x, y, \theta)\| \leq & C_{1} t^{l}(r(x) r(y))^{j+n} e^{-C_{2} \frac{\operatorname{dist}^{2}(x, \mathcal{V})}{t}} e^{-C_{2} \frac{\operatorname{dist}^{2}(y, \mathcal{V})}{t}} \\
& \cdot \int_{0}^{t} \int_{\mathcal{V}} s^{-\frac{d}{2}} e^{-C_{2} \frac{d^{2}(x, w)}{s}} d w d s .
\end{aligned}
$$

The last integral needs to be estimated only if $x$ belongs to some fixed relatively compact neighbourhood of $\mathcal{V}$ and here one uses geodesic coordinates around $x$ and a change of varibales to see that it is bounded. By the definition of $r$ and the hyperbolic distance, for each $j \in \mathbb{N}$, each $T>0$ and each $c>0$ there exists a constant $C>0$ such that for all $x \in X$ and all $t \in(0, T]$ one has

$$
e^{-c \frac{\operatorname{dist}^{2}(x, \mathcal{V})}{t}} r(x)^{j} \leq C .
$$

Thus for all $j, l \in \mathbb{N}, T>0$ there exists a constant $C>0$ such that for all $x, y \in X$ and all $t \in(0, T]$ one has

$$
r(x)^{j} r(y)^{j}\|P * Q(t, x, y, \theta)\| \leq C t^{l} .
$$

Using the previous results, one now immediately checks that

$$
K_{\tilde{\Delta}_{p}^{\theta}}(t, x, y):=P(t, x, y ; \theta)+P * Q(t, x, y, \theta)
$$

is the integral kernel of $e^{-t \tilde{\Delta}_{p}^{\theta}}$. This implies in particular that the kernel $K_{\tilde{\Delta}_{p}^{\theta}}$ has the same growth properties as the kernel $K_{\tilde{\Delta}_{p}^{\theta}}$ stated in Proposition 10.3, i.e. for each $T>0$ there exist constants $C_{1}, C_{2}>0$ such that for all $x, y \in X$ and all $t \in(0, T]$ and all $j \in\{0,1\}$ one can estimate

$$
\left\|\left(\tilde{\Delta}_{p}^{\theta}\right)^{j} K_{\tilde{\Delta}_{p}^{\theta}}(t, x, y)\right\| \leq C_{1} r(x)^{n} r(y)^{n} t^{-\frac{d}{2}-j} e^{-C_{2} \frac{d^{2}(x, y)}{t}} .
$$

We can now generalize the results of section 10 to the operator $\tilde{\Delta}_{p}^{\theta}$. We remark that this proposition is not a direct consequence of the results of section 10, since the operator $\tilde{\Delta}_{p}^{\theta}$ is not a differential operator. However, since outside the fixed collar where the variation takes place it is a differential operator of the form studied in section 10, it is of course not surprising that the results of that section continue to hold. We let $T_{p, u}:=T_{\nu_{p}(\rho), u}+\rho(\Omega)$ Id, where the latter operator is as in section 10. We make from now on the convention that $u>Y_{1}+2$, i.e. the auxilliary operator doesn't interact with the variation.

Proposition 13.1. In the preceding notations one has:

(1) The operator $e^{-t \tilde{\Delta}_{p}^{\theta}}-e^{-t T_{p, u}}$ is trace class and one has

$$
\operatorname{Tr}_{\mathrm{rel}, u}\left(e^{-t \tilde{\Delta}_{p}^{\theta}}\right):=\operatorname{Tr}\left(e^{-t \tilde{\Delta}_{p}^{\theta}}-e^{-t T_{p, u}}\right)=\int_{X} \operatorname{Tr}\left(K_{\tilde{\Delta}_{p}^{\theta}}(t, x, x)-K_{T_{p, u}}(t, x, x) d x\right) d x
$$


(2) There is a short-time asymptotic expansion

$$
\operatorname{Tr}_{\mathrm{rel}, u}\left(e^{-t \tilde{\Delta}_{p}^{\theta}}\right)=\sum_{j=0}^{\infty} a_{j, \theta} t^{j-\frac{d}{2}}+\sum_{j=0}^{\infty} b_{j, \theta} t^{j-\frac{1}{2}} \log t+\sum_{j=0}^{\infty} c_{j, \theta} t^{j}
$$

as $t \rightarrow 0+$, where the coefficients depend smoothly on $\theta$.

(3) The essential spectrum of $\tilde{\Delta}_{p}^{\theta}$ is contained in $\left[\frac{1}{4}, \infty\right)$ and in particular the complex $\mathcal{D}_{\theta}^{*}(X ; F)$ is a Fredholm complex.

(4) For each $\theta$ there exist constants $C, c$ which depend on $\theta$ such that one has

$$
\operatorname{Tr}_{\mathrm{rel}, u}\left(e^{-t \tilde{\Delta}_{p}^{\theta}}\right)-\operatorname{dim} \operatorname{Ker}\left(\tilde{\Delta}_{p}^{\theta}\right) \leq C e^{-c t}
$$

for $t \geq 1$.

(5) The operator $e^{-t \Delta_{p}^{\theta}}-e^{-t T_{p, u}}$ is trace class and if $\operatorname{Tr}_{\mathrm{rel}, u}\left(e^{-t \Delta_{p}^{\theta}}\right):=\operatorname{Tr}\left(e^{-t \Delta_{p}^{\theta}}-e^{-t T_{p, u}}\right)$, then $\operatorname{Tr}_{\mathrm{rel}, u}\left(e^{-t \Delta_{p}^{\theta}}\right)=\operatorname{Tr}_{\mathrm{rel}, u}\left(e^{-t \tilde{\Delta}_{p}^{\theta}}\right)$.

Proof. Let $P$ be as in (13.4) . Then the function $K_{\Delta_{p}}(t, x, y)-P(t, x, y ; \theta)$ vanishes if $(x, y) \notin X\left(Y_{1}+1\right) \times X \cup X \times X\left(Y_{1}+1\right)$; let us recall that $X\left(Y_{1}+1\right)$ is compact. Thus using an estimate analogous to (13.13), (2.17) and Proposition 10.3 imply that for each $j \in \mathbb{N}$ the function

$$
(r(x) r(y))^{j}\left(K_{\Delta_{p}}(t, x, y)-P(t, x, y ; \theta)\right)
$$

is square integrable over $X \times X$. Thus, by (13.14) and (13.15), for each $j \in \mathbb{N}$ the function

$$
(r(x) r(y))^{j}\left(K_{\Delta_{p}}(t, x, y)-K_{\tilde{\Delta}_{p}^{\theta}}(t, x, y)\right)
$$

is square-integrable over $X \times X$, i.e. the operators $M_{r^{j}} \circ\left(e^{-t \Delta_{p}}-e^{-t \tilde{\Delta}_{p}^{\theta}}\right)$ and $\left(e^{-t \Delta_{p}}-\right.$ $\left.e^{-t \tilde{\Delta}_{p}^{\theta}}\right) \circ M_{r^{j}}$ are Hilbert - Schmidt operators, where $M_{r^{j}}$ denotes the operator induced by multiplication with the function $r^{j}$. If we apply (2.17), (11.1), Proposition 10.3 and (13.16), we see that for $j \in \mathbb{N}$ sufficiently large the functions $(r(y))^{-j} K_{\Delta_{p}}(t, x, y)$ and $(r(x))^{-j} K_{\tilde{\Delta}_{p}^{\theta}}(t, x, y)$ are square integrable over $X \times X$ i.e. $e^{-t \Delta_{p}} \circ M_{r^{-j}}$ and $M_{r^{-j}} \circ e^{-t \tilde{\Delta}_{p}^{\theta}}$ are Hilbert Schmidt operators. Put $\tau=\frac{t}{2}$. Then one has

$$
\begin{aligned}
e^{-t \Delta_{p}}-e^{-t \tilde{\Delta}_{p}^{\theta}} & =\left(e^{-\tau \Delta_{p}} \circ M_{r^{-j}}\right) \circ\left(M_{r^{j}} \circ\left(e^{-\tau \Delta_{p}}-e^{-\tau \tilde{\Delta}_{p}^{\theta}}\right)\right) \\
& +\left(\left(e^{-\tau \Delta_{p}}-e^{-\tau \tilde{\Delta}_{p}^{\theta}}\right) \circ M_{r^{j}}\right) \circ\left(M_{r^{-j}} e^{-\tau \tilde{\Delta}_{p}^{\theta}}\right)
\end{aligned}
$$

and thus $e^{-t \Delta_{p}}-e^{-t \tilde{\Delta}_{p}^{\theta}}$ is trace class and by [Wa, Theorem 4.1] one has

$$
\operatorname{Tr}\left(e^{-t \Delta_{p}}-e^{-t \tilde{\Delta}_{p}^{\theta}}\right)=\int_{X}\left(\operatorname{Tr} K_{\Delta_{p}}(t, x, x)-\operatorname{Tr} K_{\tilde{\Delta}_{p}^{\theta}}(t, x, x)\right) d x .
$$

Applying Proposition 10.7, (11) follows. By (13.14) and the fact that $X$ has finite volume, for the short time asymptotic expansion of the relative trace we can replace the kernel $K_{\tilde{\Delta}_{p}^{\theta}}$ 
by its parametrix $P$, i.e. we have

$$
\begin{aligned}
\operatorname{Tr}_{\mathrm{rel}, u}\left(e^{-t \tilde{\Delta}_{p}^{\theta}}\right) & =\int_{X} \operatorname{Tr}\left(P(t, x, x, \theta)-K_{T_{p, u}}(t, x, x)\right) d x+O\left(t^{\infty}\right) \\
& =\int_{X} \Psi_{1}(x) \operatorname{Tr} E_{t}^{p, \theta}(t, x, x) d x+\int_{X}\left(\Psi_{2}(x)-1\right) \operatorname{Tr} K_{\Delta_{p}}(t, x, x) d x \\
& +\int_{X}\left(\operatorname{Tr} K_{\Delta_{p}}(t, x, x)-\operatorname{Tr} K_{T_{p, u}}(t, x, x)\right)+O\left(t^{\infty}\right)
\end{aligned}
$$

as $t \rightarrow+0$. The first two integrals in the last equation range over compact subsets of $X$ only. Moreover, using (12.8), (13.1) and the pointwise short time asymptotic expansion of $E_{t}^{p}$ [Gi], it follows that the first integral has the required short time asymptotic expansion (without logarithmic terms) and that the coefficients in this expansion depend smoothly on $\theta$. Also, by the pointwise heat asymptotics of $\operatorname{Tr} K_{\Delta_{p}}(t, x, x)$ [Gi], the second summand has the required asymptotics without logarithmic terms. The third summand has the required asymptotic expansion by Proposition 10.9. This proves (21). (3) follows from the trace class property of $e^{-t \Delta_{p}}-e^{-t \tilde{\Delta}_{p}^{\theta}}$, Proposition 10.10 and the invaraince of the essential spectrum under compact perturbations. (4) follows in exactly the same way as the corresponding statement in Proposition 10.10. Since $\Phi_{\theta}$ commutes with the operator $T_{p, u}$, (5) follows.

Remark 13.2. For $\theta \in\left(0, \frac{\pi}{2}\right)$ one has in fact $\operatorname{dim} \operatorname{Ker}\left(\tilde{\Delta}_{p}^{\theta}\right)=0$ as we will later show using Lesch's argument. However, for $\theta=0$ this is not the case by (12.4) and the results of section 8. The possibility of such a jump in the dimension of the cohomology has been predicted by Lesch [Le, section 5.2.3].

The previous proposition and standard results on Mellin transforms [Gi] imply in particular that we can define the relative analytic torsion

$$
T_{\mathrm{rel}, u}^{\theta}\left(X, g_{1}, h_{1} ; E_{\rho}\right)
$$

by using the relative traces $\operatorname{Tr}_{\mathrm{rel}, u}\left(e^{-t \Delta_{p}^{\theta}}\right)$ and the corresponding relative zeta functions in the same way as at the end of section 10. We have put $g_{1}$ and $h_{1}$ in the notation here in order to indicate that we still work with the perturbed metrics $g_{1}$ and $h_{1}$.

Now for $Y>Y_{1}+1$ we consider the manifold $M(Y)$ from equation (11.2). This manifold contains $X^{+}$and also the collar $W$. Also, by construction, there exists a metric on $M(Y)$ which coincides with the metric $g_{1}$ on $X(Y)$ and there exists a Hermitian flat vector bundle $F$ over $M(Y)$ whose restriction to $X(Y) \subset M(Y)$ is isometric to the bundle $F$ with the metric $h_{1}$. Therefore, if we let $M(Y)^{+}:=X^{+}, M(Y)^{-}:=M(Y)-M(Y)^{+}$, then we are in a special case of a situation which is considered by Lesch. We shall denote the corresponding operators on $M(Y)$ over the flat bundle $F$ by $\Delta_{p}^{\theta}[M(Y)] \operatorname{resp.} \tilde{\Delta}_{p}^{\theta}[M(Y)]$. Let $e^{-t \tilde{\Delta}_{p}^{\theta}}$ and $e^{-\tilde{\Delta}_{p}^{\theta}[M(Y)]}$ denote the heat semigroups of $\tilde{\Delta}_{p}^{\theta}$ and $\tilde{\Delta}_{p}^{\theta}[M(Y)]$. We now show that the kernel of $e^{-t \tilde{\Delta}_{p}^{\theta}}$ can be approximated by the kernel of $e^{-t \tilde{\Delta}_{p}^{\theta}[M(Y)]}$ on the fixed collar $W$ if $Y \rightarrow \infty$ in the same way as in Proposition 11.2, which we can again not apply directly since $T$ and thus the operators $\tilde{\Delta}_{p}^{\theta}$ are not differential operators. 
Proposition 13.3. Let $D$ be a differential operator acting on the smooth F-valued p-forms. Then one has uniformly in $x_{0}, y_{0} \in X\left(Y_{1}\right)$ :

$$
\frac{d^{i}}{(d t)^{i}} D_{x} K_{\tilde{\Delta}_{p}^{\theta}}\left(t, x_{0}, y_{0}\right)=\lim _{Y \rightarrow \infty} \frac{d^{i}}{(d t)^{i}} D_{x} K_{\tilde{\Delta}_{p}^{\theta}[M(Y)]}\left(t, x_{0}, y_{0}\right)
$$

where $D_{x}$ indicates that $D$ is applied to the first variable and where $i \in \mathbb{N}$.

Proof. Let $K_{\Delta_{p}[M(Y)]}$ be the integral kernel of $e^{-t \Delta_{p}[M(Y)]}$ and let $E_{t}^{p, \theta}$ be as above. Then one constructs the kernel $K_{\tilde{\Delta}_{p}^{\theta}[M(Y)]}$ as above. More precisely, let $\Phi_{1}$ and $\Psi_{1}$ be the same functions as above. Then $\Phi_{1}$ and $\Psi_{1}$ can also be regarded as functions on $M(Y)$. Also, $\Phi_{2}$ and $\Psi_{2}$ are equal to 1 in a neighbourhood of $\partial X(Y)$ in $X$. Therefore, they may be extended by 1 to functions on $M(Y)$ and denoting these functions by the same symbol, it follows as before that

$$
P^{Y}(t, x, y ; \theta):=\Phi_{1}(x) \tilde{E}_{t}^{p, \theta} \Psi_{1}(y)+\Phi_{2}(x) K_{\Delta_{p}[M(Y)]} \Psi_{2}(y)
$$

is a parametrix for the operator $\tilde{\Delta}_{p}^{\theta}[M(Y)]$, where $\tilde{E}_{t}^{p, \theta}$ is as in (13.2). As before let

$$
Q_{1}^{Y}(t, x, y ; \theta):=\left(\frac{d}{d t}+\tilde{\Delta}_{p}^{\theta}[M(Y)]\right) P^{Y}(t, x, y ; \theta)
$$

where $\tilde{\Delta}_{p}^{\theta}[M(Y)]$ is applied to the $x$-variable and define inductively

$$
Q_{k+1}^{Y}(t, x, y ; \theta):=\int_{0}^{t} \int_{M(Y)} Q_{1}^{Y}(t-s, x, w ; \theta) \circ Q_{k}^{Y}(s, w, y ; \theta) d w d s
$$

and then let

$$
Q^{Y}(t, x, y ; \theta):=\sum_{k=1}^{\infty}(-1)^{k} Q_{k}^{Y}(t, x, y ; \theta)
$$

Then

$$
K_{\tilde{\Delta}_{p}^{\theta}[M(Y)]}(t, x, y):=P^{Y}(t, x, y ; \theta)+\int_{0}^{t} \int_{M(Y)} P^{Y}(t-s, x, w ; \theta) \circ Q^{Y}(s, w, y ; \theta) d w d s
$$

is the integral kernel of $\tilde{\Delta}_{p}^{\theta}[M(Y)]$. For $x, y \in X(Y)$ one has

$$
P(t, x, y ; \theta)-P^{Y}(t, x, y ; \theta)=\Phi_{2}(x)\left(K_{\Delta_{p}}(t, x, y)-K_{\Delta_{p}[M(Y)]}(t, x, y)\right) \Psi_{2}(y) .
$$

Moreover, by construction one has $\tilde{\Delta}_{p}[M(Y)]=\Delta_{p}[M(Y)]=\Delta_{p}$ on $\operatorname{supp}\left(\Phi_{2}\right) \cap X(Y)$. Thus by Proposition 11.2, for $x_{0}, y_{0} \in X\left(Y_{1}+1\right)$ one can estimate

$$
\begin{aligned}
& \max \left\{\left\|P\left(t, x_{0}, y_{0} ; \theta\right)-P^{Y}\left(x_{0}, y_{0} ; \theta\right)\right\|,\left\|Q_{1}\left(t, x_{0}, y_{0} ; \theta\right)-Q_{1}^{Y}\left(x_{0}, y_{0} ; \theta\right)\right\|\right\} \\
& \quad \leq C_{1} e^{-C_{2} \frac{\operatorname{dist}^{2}\left(X\left(Y_{1}+1\right), \partial X(Y)\right)}{t}},
\end{aligned}
$$


where $P\left(t, x_{0}, y_{0} ; \theta\right)$ and $Q_{1}\left(t, x_{0}, y_{0}, \theta\right)$ are as in (13.4) resp. (13.5). The inner integrals in (13.8) and (13.17) range over the same compact set $\mathcal{V} \subset X\left(Y_{1}+1\right)$ and by induction it follows that one has

$$
\left\|Q_{k+1}\left(t, x_{0}, y_{0} ; \theta\right)-Q_{k+1}^{Y}\left(t, x_{0}, y_{0} ; \theta\right)\right\| \leq C_{1} e^{-C_{2} \frac{\operatorname{dist}^{2}\left(X\left(Y_{1}+1\right), \partial X(Y)\right)}{t}} \frac{t^{k}}{k !},
$$

for all $x_{0}, y_{0} \in X\left(Y_{1}+1\right)$, where $Q_{k+1}\left(s, x_{0}, y_{0} ; \theta\right)$ is as in (13.8). Thus one has

$$
\left\|Q\left(t, x_{0}, y_{0} ; \theta\right)-Q^{Y}\left(t, x_{0}, y_{0} ; \theta\right)\right\| \leq C_{1} e^{-C_{2} \frac{\operatorname{dist}^{2}\left(X\left(Y_{1}+1\right), \partial X(Y)\right)}{t}},
$$

where $Q\left(s, x_{0}, y_{0} ; \theta\right)$ is as in (13.10). Writing

$$
\begin{aligned}
& K_{\tilde{\Delta}_{p}^{\theta}}(t, x, y)-K_{\tilde{\Delta}_{p}^{\theta}[M(Y)]}(t, x, y)=P(t, x, y ; \theta)-P^{Y}(t, x, y ; \theta) \\
+ & \int_{0}^{t} \int_{\mathcal{V}}\left(P(t-s, x, w ; \theta)-P^{Y}(t-s, x, w ; \theta)\right) Q(s, w, y) d w d s \\
+ & \int_{0}^{t} \int_{\mathcal{V}} P^{Y}(t-s, x, w ; \theta)\left(Q(s, w, y ; \theta)-Q^{Y}(s, w, y ; \theta)\right) d w d s
\end{aligned}
$$

and employing the previous estimates, the Proposition is proved if $D$ is the identity. For general $D$ resp. for the $t$-derivatives, the proof is word for word the same since the results of Proposition 11.2 hold for general $D$ resp. the $t$-derivatives.

\section{The FIRST VERSION OF THE GLUING FORMULA}

In this section we prove the gluing formula under the additional assumption that we work with the metrics $g_{1}$ and $h_{1}$ which are compact perturbations of our original metric but which are of product structure on the part of the manifold where the variation of the De Rham complex takes place. We keep all the notations of the preceding section and we remark that by (5) in Proposition 13.1 also in our case we may interchange $\tilde{\Delta}_{p}^{\theta}$ with $\Delta_{p}^{\theta}$ if we consider relative traces. We start with the following proposition.

Proposition 14.1. The kernel $K_{\tilde{\Delta}_{p}^{\theta}}(t, x, y)$ is differentiable in $\theta$ and if $\frac{d}{d \theta} e^{-t \tilde{\Delta}_{p}^{\theta}}$ denotes the integral operator with kernel $\frac{d}{d \theta} K_{\tilde{\Delta}_{p}^{\theta}}(t, x, y)$, then $\frac{d}{d \theta} e^{-t \tilde{\Delta}_{p}^{\theta}}$ is trace class. Also $\operatorname{Tr}_{\mathrm{rel}, u}\left(e^{-t \tilde{\Delta}_{p}^{\theta}}\right)$ is differentiable in $\theta$. One has

$$
\operatorname{Tr}\left(\frac{d}{d \theta} e^{-t \tilde{\Delta}_{p}^{\theta}}\right)=\int_{X} \operatorname{Tr}\left(\frac{d}{d \theta} K_{\tilde{\Delta}_{p}^{\theta}}(t, x, x)\right) d x=\frac{d}{d \theta} \operatorname{Tr}_{\mathrm{rel}, u}\left(e^{-t \tilde{\Delta}_{p}^{\theta}}\right),
$$

Moreover, one has

$$
\operatorname{Tr}\left(\frac{d}{d \theta} e^{-t \tilde{\Delta}_{p}^{\theta}}\right)=-t \operatorname{Tr}\left(\left(\frac{d}{d \theta} \tilde{\Delta}_{p}^{\theta}\right) e^{-t \tilde{\Delta}_{p}^{\theta}}\right)=-t \int_{X} \operatorname{Tr}\left(\left(\frac{d}{d \theta} \tilde{\Delta}_{p}^{\theta}\right) K_{\tilde{\Delta}_{p}^{\theta}}(t, x, x)\right) d x
$$

where $\frac{d}{d \theta} \tilde{\Delta}_{p}^{\theta}$ is applied to the first variable of $K_{\tilde{\Delta}_{p}^{\theta}}(t, x, x)$. 
Proof. Let $\tilde{E}_{t}^{p, \theta}(x, y)$ be as in (13.2) and let $P(t, x, y ; \theta)$ be as in (13.4). Then (12.8), (12.9), (13.1) and and the Gaussian bounds for $E_{t}^{p}$ immediately show that $P(t, x, y ; \theta)$ is differentiable in $x, y, t$ and $\theta$ and that for each $T>0$ there exist constants $C_{1}, C_{2}>0$ such that for all $t \in(0, T]$, all $\theta$ and all $x, y \in X$ one has

$$
\left\|\frac{d}{d \theta} P(t, x, y ; \theta)\right\|=\left\|\Phi_{1}(x) \frac{d}{d \theta} \tilde{E}_{t}^{p, \theta}(x, y) \Psi_{1}(y)\right\| \leq C_{1} t^{-\frac{d}{2}} e^{-C_{2} \frac{d^{2}(x, y)}{t}} .
$$

and

$$
\begin{aligned}
& \left\|\left(\frac{d}{d t}+\tilde{\Delta}_{p}^{\theta}\right) \frac{d}{d \theta} P(t, x, y ; \theta)\right\|=\left\|\left(\frac{d}{d t}+\tilde{\Delta}_{p}^{\theta}\right) \Phi_{1}(x) \frac{d}{d \theta} \tilde{E}_{t}^{p, \theta}(x, y) \Psi_{1}(y)\right\| \\
& \leq C_{1} t^{-\frac{d}{2}-1} e^{-C_{2} \frac{d^{2}(x, y)}{t}} .
\end{aligned}
$$

Moreover, one has $\frac{d}{d \theta} \tilde{\Delta}_{p}^{\theta}=0$ on $\operatorname{supp}\left(\Phi_{2}\right)$. Therefore, one has

$$
\left\|\left(\frac{d}{d \theta} \tilde{\Delta}_{p}^{\theta}\right) P(t, x, y ; \theta)\right\|=\left\|\left(\frac{d}{d \theta} \tilde{\Delta}_{p}^{\theta}\right) \Phi_{1}(x) \tilde{E}_{t}^{p, \theta}(x, y) \Psi_{1}(y)\right\| \leq C_{1} t^{-\frac{d}{2}-1} e^{-C_{2} \frac{d^{2}(x, y)}{t}} .
$$

This implies that $Q_{1}(t, x, y ; \theta)$, defined as in (13.5), is differentiable in $\theta$. By (13.6) one has $\frac{d}{d \theta} Q_{1}(t, x, y ; \theta)=0$ if $d(x, y)<\epsilon$. Thus the previous two estimates imply that for each $l \in \mathbb{N}$ and $T>0$ there exist constants $C_{1}, C_{2}>0$ such that for all $t \in(0, T]$, all $\theta \in \mathbb{R}$ and all $x, y \in X$ one has

$$
\begin{aligned}
\left\|\frac{d}{d \theta} Q_{1}(t, x, y ; \theta)\right\|= & \left\|\left(\frac{d}{d \theta} \tilde{\Delta}_{p}^{\theta}\right) P(t, x, y ; \theta)+\left(\frac{d}{d t}+\tilde{\Delta}_{p}^{\theta}\right)\left(\frac{d}{d \theta} P(t, x, y ; \theta)\right)\right\| \\
& \leq C_{1} t^{l} e^{-C_{2} \frac{d^{2}(x, y)}{t}} .
\end{aligned}
$$

Thus one shows by induction that each $Q_{k}(t, x, y ; \theta)$, defined as in (13.8), is differentiable in $\theta$ with

$$
\left\|\frac{d}{d \theta} Q_{k}(t, x, y ; \theta)\right\|=\leq C_{1} t^{l} \frac{t^{k}}{k !} e^{-C_{2} \frac{d^{2}(x, y)}{t}},
$$

where the constants $C_{1}, C_{2}$ are independent of $\theta$. This implies that $Q(t, x, y ; \theta)$, defined as in (13.10) is differentiable in $\theta$ and that

$$
\left\|\frac{d}{d \theta} Q(t, x, y ; \theta)\right\|=\leq C_{1} t^{l} e^{-C_{2} \frac{d^{2}(x, y)}{t}}
$$

for all $\theta \in \mathbb{R}$, all $x, y \in X$. Arguing as in the previous section before (13.14) and applying (14.1) it follows that for each $l$ there exists a constant $C$ such that

$$
\left\|\frac{d}{d \theta} P * Q(t, x, y ; \theta)\right\| \leq C t^{l}
$$


for all $\theta \in \mathbb{R}$, all $x, y \in X$. Thus together with (13.15) and (14.1), it follows that the kernel $\frac{d}{d \theta} K_{\tilde{\Delta}_{p}^{\theta}}(t, x, y)$ is differentiable in $\theta$ and that that

$$
\left\|\frac{d}{d \theta} K_{\tilde{\Delta}_{p}^{\theta}}(t, x, y)\right\| \leq C_{1} t^{-\frac{d}{2}} e^{-C_{2} \frac{d^{2}(x, y)}{t}}
$$

for all $\theta \in \mathbb{R}, x, y \in X, t \in(0, T]$. By construction, $\frac{d}{d \theta} K_{\tilde{\Delta}_{p}^{\theta}}(t, x, y)$ vanishes if $\Phi_{2}(x)=0$, in particular, it has uniform compact $x$-support. Thus if $r$ is the function introduced in section 10, then together with (2.17) and the estimate analogous to (13.13) it follows that $r(x)^{j} r(y)^{j} \frac{d}{d \theta} K_{\tilde{\Delta}_{p}^{\theta}}(t, x, y)$ is square-integrable over $X \times X$ for each $j \in \mathbb{N}$, i.e. the operators $\frac{d}{d \theta} K_{\tilde{\Delta}_{p}^{\theta}}(t, x, y) \circ M_{r^{j}}$ and $M_{r^{j}} \circ \frac{d}{d \theta} K_{\tilde{\Delta}_{p}^{\theta}}(t, x, y)$ are Hilbert Schmidt operators, where $M_{r^{j}}$ is again the operator induced by multiplication with the function $r^{j}$. As in the proof of Proposition 13.1, for $j \in \mathbb{N}$ sufficiently large the operators $M_{r^{-j}} \circ e^{-t \tilde{\Delta}_{p}^{\theta}}$ and $e^{-t \tilde{\Delta}_{p}^{\theta}} \circ M_{r^{-j}}$ are Hilbert Schmidt operators and thus putting $\tau:=t / 2$ and writing

$$
\frac{d}{d \theta} e^{-t \tilde{\Delta}_{p}^{\theta}}=\left(\frac{d}{d \theta} e^{-\tau \tilde{\Delta}_{p}^{\theta}} \circ M_{r^{j}}\right) \circ\left(M_{r^{-j}} e^{-\tau \tilde{\Delta}_{p}^{\theta}}\right)+\left(e^{-\tau \tilde{\Delta}_{p}^{\theta}} \circ M_{r^{-j}}\right) \circ\left(M_{r^{j}} \circ \frac{d}{d \theta} e^{-\tau \tilde{\Delta}_{p}^{\theta}}\right),
$$

it follows that $\frac{d}{d \theta} e^{-t \tilde{\Delta}_{p}^{\theta}}$ is trace class. Applying Proposition 10.7 and [Wa, Theorem 4.1], the first equation now follows easily.

To prove the second equation, we remark that as in [MuS, (6.11)], for $\theta, \theta_{1} \in \mathbb{R}$ we have

$$
e^{-t \tilde{\Delta}_{p}^{\theta_{1}}}-e^{-t \tilde{\Delta}_{p}^{\theta}}=-\int_{0}^{t} \frac{d}{d s}\left(e^{-(t-s) \tilde{\Delta}_{p}^{\theta_{1}}} \circ e^{-s \tilde{\Delta}_{p}^{\theta}}\right) d s=-\int_{0}^{t} e^{-(t-s) \tilde{\Delta}_{p}^{\theta_{1}}}\left(\tilde{\Delta}_{p}^{\theta_{1}}-\tilde{\Delta}_{p}^{\theta}\right) e^{-s \tilde{\Delta}_{p}^{\theta}}
$$

in the strong operator topology and therefore by (12.10), one has

$$
\frac{d}{d \theta} e^{-t \tilde{\Delta}_{p}^{\theta}}=-\int_{0}^{t} e^{-(t-s) \tilde{\Delta}_{p}^{\theta}} \circ\left(\frac{d}{d \theta} \tilde{\Delta}_{p}^{\theta}\right) \circ e^{-s \tilde{\Delta}_{p}^{\theta}} d s
$$

in the strong operator topology. Since the operator $\left(\frac{d}{d \theta} \tilde{\Delta}_{p}^{\theta}\right)$ is compactly supported, the previous construction of $K_{\tilde{\Delta}_{p}^{\theta}}$ shows that proceeding analogously to the proof of Proposition 11.1 one can establish that the operators $\left(\frac{d}{d \theta} \tilde{\Delta}_{p}^{\theta}\right) \circ e^{-t \tilde{\Delta}_{p}^{\theta}}$ and $e^{-t \tilde{\Delta}_{p}^{\theta}} \circ\left(\frac{d}{d \theta} \tilde{\Delta}_{p}^{\theta}\right)$ are trace class and that their trace norm is uniformly bounded for $t$ in compat subsets of $(0, \infty)$. Therefore, splitting the integral in (14.3) in the integral from $(0, t / 2)$ and $(t / 2, t)$, we obtain

$$
\begin{aligned}
\operatorname{Tr}\left(\frac{d}{d \theta} e^{-t \tilde{\Delta}_{p}^{\theta}}\right) & =-\int_{0}^{t} \operatorname{Tr}\left(e^{-(t-s) \tilde{\Delta}_{p}^{\theta}} \circ\left(\frac{d}{d \theta} \tilde{\Delta}_{p}^{\theta}\right) \circ e^{-s \tilde{\Delta}_{p}^{\theta}}\right) d s \\
& =-\int_{0}^{t} \operatorname{Tr}\left(\left(\frac{d}{d \theta} \tilde{\Delta}_{p}^{\theta}\right) \circ e^{-s \tilde{\Delta}_{p}^{\theta}} \circ e^{-(t-s) \tilde{\Delta}_{p}^{\theta}}\right) d s=-t \operatorname{Tr}\left(\left(\frac{d}{d \theta} \tilde{\Delta}_{p}^{\theta}\right) e^{-t \tilde{\Delta}_{p}^{\theta}}\right) .
\end{aligned}
$$

which is the first equality of the second equation in the proposition. The second equality of this equation follows again from [Wa, Theorem 4.1]. 
Remark 14.2. Let us remark that without the trace the second equation of the proposition would not hold necessarily, since the operators under the integral in (14.3) do not commute in general.

Let $\beta_{\theta}$ be as in (12.6). Using (13.16) and proceeding exactly as in the proof of Proposition 11.1, one can show that $\beta_{\theta}\left(e^{-t \Delta_{p}^{\theta}}\right)$ is trace class. We can now prove the analog of [Le, Theorem 5.3]. We point out that in our understanding Lesch's assumption of discrete dimension spectrum is essential in his proof of that theorem, in particular in the computations yielding [Le, (5.17), (5.18)]. Therefore, we do not generalize Lesch's proof, but we reduce our statement to his statement by approximating the variation by variations on closed manifolds. Let us furthermore recall that in our situation the Euler-characteristics of $X^{+}$resp. of $\partial X^{+}$vanish.

Proposition 14.3. For $0<\theta<\frac{\pi}{2}$ the function $\theta \mapsto \operatorname{Tr}_{\mathrm{rel}, u}\left(e^{-t \Delta_{p}^{\theta}}\right)$ is differentiable in $\theta$ and one has

$$
\frac{d}{d \theta}\left(\sum_{p}(-1)^{p} p \operatorname{Tr}_{\mathrm{rel}, u}\left(e^{-t \Delta_{p}^{\theta}}\right)\right)=-t \frac{d}{d t} \frac{4}{\sin 2 \theta}\left(\sum_{p}(-1)^{p} \operatorname{Tr}\left(\beta_{\theta} e^{-t \Delta_{p}^{\theta}}\right)\right) .
$$

Furthermore, one has

$$
\sum_{p}(-1)^{p} \operatorname{Tr}\left(\beta_{\theta} e^{-t \Delta_{p}^{\theta}}\right)=O\left(t^{\infty}\right)
$$

as $t \rightarrow 0+$.

Proof. For $Y>Y_{1}+1$ we let $M(Y)$ be the same manifold as in (11.2). As in the previous section in the paragraph before Proposition 13.3, we can perform the corresponding variation of the De Rham complex on $M(Y)$ an consider the operators $\Delta_{p}^{\theta}[M(Y)] \operatorname{resp.} \tilde{\Delta}_{p}^{\theta}[M(Y)]$ as well as their heat kernels. By construction, the operators $\frac{d}{d \theta} \tilde{\Delta}_{p}^{\theta}$ and $\frac{d}{d \theta} \tilde{\Delta}_{p}^{\theta}[M(Y)]$ agree on their support which lies in $X\left(Y_{1}+1\right)$. Thus by Proposition 13.3, one has

$$
\int_{X} \operatorname{Tr}\left(\left(\frac{d}{d \theta} \tilde{\Delta}_{p}^{\theta}\right) K_{\tilde{\Delta}_{p}^{\theta}}(t, x, x)\right) d x=\lim _{Y \rightarrow \infty} \int_{X} \operatorname{Tr}\left(\left(\frac{d}{d \theta} \tilde{\Delta}_{p}^{\theta}[M(Y)]\right) K_{\tilde{\Delta}_{p}^{\theta}[M(Y)]}(t, x, x)\right) d x
$$

where the operators are applied to the fist variable. We remark that (12.9) immediately shows that we can apply Proposition 13.3, although $T$, which occurs in $(d / d \theta) \tilde{\Delta}_{p}^{\theta}$, is not a differential operator. By (5) in Proposition 13.1, by Proposition 14.1 and by the analogous statement for the closed manifold $M[Y]$, the last equality is equivalent to the equality

$$
\frac{d}{d \theta} \operatorname{Tr}_{\mathrm{rel}, u}\left(e^{-t \Delta_{p}^{\theta}}\right)=\lim _{Y \rightarrow \infty} \frac{d}{d \theta} \operatorname{Tr}\left(e^{-t \Delta_{p}^{\theta}[M(Y)]}\right)
$$


Now we apply [Le, Theorem 5.3, (5.9)] to the closed manifold $M(Y)$. This gives:

$$
\begin{aligned}
\frac{d}{d \theta}\left(\sum_{p}(-1)^{p} p \operatorname{Tr}_{\mathrm{rel}, u}\left(e^{-t \Delta_{p}^{\theta}}\right)\right) & =\lim _{Y \rightarrow \infty} \frac{d}{d \theta}\left(\sum_{p}(-1)^{p} p \operatorname{Tr}\left(e^{-t \Delta_{p}^{\theta}[M(Y)]}\right)\right) \\
& =\lim _{Y \rightarrow \infty}-t \frac{d}{d t} \frac{4}{\sin 2 \theta}\left(\sum_{p}(-1)^{p} \operatorname{Tr}\left(\beta_{\theta} e^{-t \Delta_{p}^{\theta}[M(Y)]}\right)\right) \\
& =\lim _{Y \rightarrow \infty}-t \frac{d}{d t} \frac{4}{\sin 2 \theta} \sum_{p}(-1)^{p} \int_{X^{+}} \operatorname{Tr} K_{\Delta_{p}^{\theta}[M(Y)]}(t, x, x) d x
\end{aligned}
$$

By Proposition 13.3, which by (12.8) clearly also holds if we replace $\tilde{\Delta}_{p}^{\theta}$ by $\Delta_{p}^{\theta}$, one has

$$
\begin{aligned}
& \lim _{Y \rightarrow \infty}-t \frac{d}{d t} \frac{4}{\sin 2 \theta} \sum_{p}(-1)^{p} \int_{X^{+}} \operatorname{Tr} K_{\Delta_{p}^{\theta}[M(Y)]}(t, x, x) d x \\
= & -t \frac{d}{d t} \frac{4}{\sin 2 \theta} \sum_{p}(-1)^{p} \int_{X^{+}} \operatorname{Tr} K_{\Delta_{p}^{\theta}}(t, x, x) d x \\
= & -t \frac{d}{d t} \frac{4}{\sin 2 \theta}\left(\sum_{p}(-1)^{p} \operatorname{Tr}\left(\beta_{\theta} e^{-t \Delta_{p}^{\theta}}\right)\right) .
\end{aligned}
$$

This proves the first equation of the proposition. To prove the second one, one can proceed exactly as Lesch in the proof of [Le, equation 5.10] on page 27-28: By (13.4), (13.14) and (13.15) one has

$$
\int_{X^{+}} \operatorname{Tr}\left(K_{\Delta_{p}}(t, x, x)\right) d x=\int_{X^{+}} \operatorname{Tr} E_{t}^{p, \theta}(x, x) d x+O\left(t^{\infty}\right),
$$

as $t \rightarrow 0+$. Using the computations of Lesch just cited, the second equation follows, since $X^{+}$resp. $\partial X^{+}$have vanishing Euler characteristic.

Now we consider the contribution of the cohomology. We recall that our bundle $F$ was the bundle $E_{\rho}, \rho \in \operatorname{Rep}(G), \rho \neq \rho_{\theta}$ from section 3. By Proposition 12.2 resp. Proposition 13.1, 3, the sequence (12.6) is a short exact sequence of Fredholm complexes. Thus by Proposition 12.1 the cohomology spaces of each complex in (12.6) are finite-dimensional and are canonically isomorphic to the kernels of the corresponding Laplacians. We equip the cohomology spaces with the inner products which are induced by this isomorphism and the $L^{2}$-inner products on the harmonic forms corresponding to the metrics $g_{1}$ and $h_{1}$. We let $\tau\left(\mathcal{H}_{\theta}\left(\left(X^{-}, Z\right), X, X^{+} ; g_{1}, h_{1}, E_{\rho}\right)\right)$ be the torsion of the long exact cohomology sequence of (12.6), where the torsion of a long exact sequence of finite-dimensional Hilbert spaces is defined as in [Le, section 2.2]. We have put $g_{1}$ and $h_{1}$ in the notation in order to emphasize that we still work with these metrics which are compactly supported perturbations of our original metrics. 
Proposition 14.4. For $0<\theta \leq \frac{\pi}{2}$, the cohomology groups $H_{\theta}^{*}\left(X ; E_{\rho}\right)$ of the complex $\mathcal{D}^{*}\left(X ; E_{\rho}\right)$ vanish. Moreover, the map

$$
\theta \mapsto \log \tau\left(\mathcal{H}_{\theta}\left(\left(X^{-}, Z\right), X, X^{+} ; g_{1}, h_{1}, E_{\rho}\right)\right)
$$

is differentiable and one has

$$
\frac{d}{d \theta} \log \tau\left(\mathcal{H}_{\theta}\left(\left(X^{-}, Z\right), X, X^{+} ; E_{\rho}\right)\right)=-\frac{2}{\sin (2 \theta)}\left[\sum_{j \geq 0}(-1)^{j} \operatorname{Tr}\left(\beta_{\theta} \mid H_{\theta}^{j}\left(X ; E_{\rho}\right)\right]=0\right.
$$

Proof. Since all complexes involved are Freholm complexes, one can just insert Lesch's arguments [Le, section 5.2.2] without any change. Thus for $0<\theta, \theta^{\prime}<\frac{\pi}{2}$ there is a chain isomorphism $\Phi_{\theta, \theta^{\prime}}$ between the short exact sequences in (12.6) corresponding to $\theta$ and $\theta^{\prime}$, [Le, equation (5.26)] . Hence the first statement follows from the isomorphism (12.5) and Proposition 12.2. Also the second statement can be proved using exactly the arguments of [Le, section 5.2.2]. We recall again that $\chi\left(X^{+}\right)=0$ resp. $\chi\left(\partial X^{+}\right)=0$.

Corollary 14.5. For $0<\theta<\frac{\pi}{2}$ the map $\theta \mapsto \log T_{\mathrm{rel} ; u}^{\theta}\left(X, g_{1}, h_{1} ; E_{\rho}\right)$ is differentiable and one has

$$
\frac{d}{d \theta} \log T_{\mathrm{rel} ; u}^{\theta}\left(X, g_{1}, h_{1} ; E_{\rho}\right)=\frac{2}{\sin 2 \theta}\left[-\sum_{j \geq 0}(-1)^{j} \operatorname{Tr}\left(\beta_{\theta} \mid H_{j}^{\theta}\left(X ; E_{\rho}\right)\right]=0 .\right.
$$

Proof. Proposition 13.1 and the first statement of the previous Proposition 14.4 imply that all the assumptions are verified to carry over equation (2.20) in [Le, Proposition 2.4] to the present case and the corollary follows from Proposition 14.3.

Proposition 14.6. The map

$$
\theta \mapsto \log \tau\left(\mathcal{H}_{\theta}\left(\left(X^{-}, Z\right), X, X^{+} ; E_{\rho}\right)\right)-\log T_{\text {rel } ; u}^{\theta}\left(X, g_{1}, h_{1} ; E_{\rho}\right)
$$

is differentiable for $0 \leq \theta \leq \frac{\pi}{2}$.

Proof. We recall that by Proposition 13.1 all operators $\Delta_{p}^{\theta}$ have pure point spectrum in $[0,1 / 4)$ and that by Propopsition 12.1 the kernels $\operatorname{Ker}\left(\Delta_{p}^{\theta}\right)$ are isomorphic to the cohomology groups of the complex $\mathcal{D}_{\theta}^{*}(X ; F)$. An inspection of Lesch's proof in [Le, section 5.2.3] of the differentiability at 0 of the expression [Le, (4.13)] shows that these two properties suffice to carry over his proof to the present situation.

We can now derive the first version of the gluing formula. We recall our notation (12.1). We let $\operatorname{det}\left(\delta_{p}^{Y_{1}}\left(g_{1}, h_{1} ; \rho\right)\right)$ denote the determinant of the matrix which represents the homomorphism $\delta_{p}: H^{p}\left(X\left(Y_{1}\right) ; E_{\rho}\right) \rightarrow H_{\min }^{p+1}\left(F_{X}\left(Y_{1}\right), \partial F_{X}\left(Y_{1}\right) ; E_{\rho}\right)$ from (12.3) with respect to $L^{2}$-orthonormal bases of harmonic forms. Here the Laplacians respectively the $L^{2}$-inner products are taken with respect to the metrics $g_{1}$ and $h_{1}$.

Theorem 14.7. Let $X$ be a hyperbolic manifold with cusps as in section 2.7. Let $\rho \in$ $\operatorname{Rep}(G), \rho \neq \rho_{\theta}$ and let $E_{\rho}$ be the corresponding flat vector bundle over $X$. Let $Y_{0}$ be as 
in section 2.7 and fix $Y_{1}>Y_{0}$. Let $g_{1}$ and $h_{1}$ be the same metrics on $X$ and $E_{\rho}$ as above. Then for $Y_{1}>Y_{0}$ and $u>Y_{1}+2$ one has

$$
\begin{aligned}
\log T_{\mathrm{rel}, u}\left(X, g_{1}, h_{1} ; E_{\rho}\right)= & \log T\left(X\left(Y_{1}\right), g_{1}, h_{1} ; E_{\rho}\right)+\log T_{\mathrm{rel}, u}\left(F_{X}\left(Y_{1}\right), \partial F_{X}\left(Y_{1}\right), g_{1}, h_{1} ; E_{\rho}\right) \\
& +\sum_{p}(-1)^{p} \log \left|\operatorname{det}\left(\delta_{p}^{Y_{1}}, g_{1}, h_{1} ; \rho\right)\right| .
\end{aligned}
$$

Proof. We proceed identically to [Le, section 6.1.1]: One has $\mathcal{D}_{\theta=\frac{\pi}{4}}\left(X ; E_{\rho}\right) \cong \mathcal{D}\left(X ; E_{\rho}\right)$ and by Proposition 12.2 , in the present case one has

$$
\left.\log \tau\left(\mathcal{H}_{\theta}\left(\left(F_{X}\left(Y_{1}\right), \partial F_{X}\left(Y_{1}\right)\right), X, X\left(Y_{1}\right) ; E_{\rho}\right)\right)\right|_{\theta=\frac{\pi}{4}}=\sum_{p}(-1)^{p} \log \left|\operatorname{det}\left(\delta_{p}^{Y_{1}}, g_{1}, h_{1} ; \rho\right)\right| .
$$

Next one has $\mathcal{D}_{\theta=0}\left(X ; E_{\rho}\right)=\mathcal{D}\left(F_{X}\left(Y_{1}\right), \partial F_{X}\left(Y_{1}\right) ; E_{\rho}\right) \oplus \mathcal{D}\left(X\left(Y_{1}\right) ; E_{\rho}\right)$. As in [Le], this implies that

$$
\left.\tau\left(\mathcal{H}_{\theta}\left(\left(F_{X}\left(Y_{1}\right), \partial F_{X}\left(Y_{1}\right)\right), X, X\left(Y_{1}\right) ; E_{\rho}\right)\right)\right|_{\theta=0}=0
$$

Thus by Proposition 14.6 one can write

$$
\begin{aligned}
& \left(\log T_{\mathrm{rel}, u}\left(X, g_{1}, h_{1} ; E_{\rho}\right)-\sum_{p}(-1)^{p} \log \left|\operatorname{det}\left(\delta_{p}^{Y_{1}}, g_{1}, h_{1} ; \rho\right)\right|\right) \\
- & \left(\log T\left(X\left(Y_{1}\right), g_{1}, h_{1} ; E_{\rho}\right)+\log T_{\mathrm{rel}, u}\left(F_{X}\left(Y_{1}\right), \partial F_{X}\left(Y_{1}\right), g_{1}, h_{1} ; E_{\rho}\right)\right) \\
= & \int_{0}^{\frac{\pi}{4}} \frac{d}{d \theta}\left(\log T_{\mathrm{rel} ; u}^{\theta}\left(X, g_{1}, h_{1} ; E_{\rho}\right)-\log \tau\left(\mathcal{H}_{\theta}\left(\left(F_{X}\left(Y_{1}\right), \partial F_{X}\left(Y_{1}\right)\right), X, X\left(Y_{1}\right) ; E_{\rho}\right)\right)\right) d \theta,
\end{aligned}
$$

and this expression is 0 by Proposition 14.4 and Corollary 14.5.

\section{THE SECOND VERSION OF THE GLUING FORMULA}

In this section we will replace the auxilliary metrics $g_{1}$ and $h_{1}$ in the gluing formula by the originial metrics $g$ and $h$. In this way the anomaly term of Brüning and Ma will appear.

We firstly prove the invariance of the relative analytic torsion under compacct pertrubations of the metric $g$ on $X$ and $h$ on $E_{\rho}$ if $\rho$ satisfies $\rho \neq \rho_{\theta}$. We recall that by the condition $\rho \neq \rho_{\theta}$ the $L^{2}$-cohomology of $X$ with coefficients in $E_{\rho}$ vanishes and that $X$ is odd-dimensional. Thus, if $X$ was a closed hyperbolic manifold, this invariance would be already known [M̈̈4, Corollary 2.7].

Proposition 15.1. Let $g_{1}$ and $h_{1}$ be compact perturbations of the metrics $g$ and $h$. Then one has

$$
T_{\mathrm{rel}, u}\left(X, g, h ; E_{\rho}\right)=T_{\mathrm{rel}, u}\left(X, g_{1}, h_{1} ; E_{\rho}\right)
$$

Proof. Let $g_{v}, v \in[0,1]$, be a smooth path of metrics on $X$ such that $g_{0}=g$, the hyperbolic metric, and assume that there exists a fixed compact subset of $X$ such that all metrics $g_{v}$ coincide outside this set. For each $p$ and $v \in[0,1]$ let $\Delta_{p}(v)$ be the corresponding flat Hodge 
Laplacian on $E_{\rho^{-v a l u e d}} p$-forms over $X$. Let $*_{v}$ be the corresponding Hodge operators, regarded as operators from $\Lambda^{p} E_{\rho}$ to $\Lambda^{d-p} E_{\rho}$, see [M̈̈4], let $\alpha(v):=\left(*_{v}^{-1}\right) \circ(d / d v) *_{v}$ and

$$
(d / d v) \Delta_{p}(v)=-\alpha(v) \delta_{p}(v) d_{p}+\delta_{p}(v) \alpha(v) d_{p}-d_{p-1} \alpha(v) \delta_{p-1}(v)+d_{p-1} \delta_{p-1}(v) \alpha(v) .
$$

The operator $(d / d v) \Delta_{p}(v)$ is a compactly supported differential operator. Therefore, $\left((d / d v) \Delta_{p}(v)\right) e^{-t \Delta_{p}(v)}$ is trace class by Proposition 11.1. Next we claim that the function $\operatorname{Tr}_{\mathrm{rel}, u}\left(e^{-t \Delta_{p}(v)}\right)$ is differentiable in $v$ and that one has

$$
(d / d v) \operatorname{Tr}_{\mathrm{rel}, u}\left(e^{-t \Delta_{p}(v)}\right)=-t \operatorname{Tr}\left(\left((d / d v) \Delta_{q}(v)\right) e^{-\Delta_{p}(v)}\right) .
$$

To prove this, one can proceed in the same way as in the proof of Proposition 14.1 and therefore we shall only indicate the main idea. Let $Y>Y_{0}+1$ such that all metrics $g_{v}$ coincide on $F_{X}(Y-1)$. Then consider the closed manifold $M(Y)$ from (11.2) which contains the set $X(Y)$. For each $v$ one can extend the metrics $g_{v}$ to metrics on $M(Y)$ and one can extend $E_{\rho}$ with its fibre metric to a flat bundle over $M(Y)$. Let $\Delta_{p}^{v}[M(Y)]$ be the corresponding flat Hodge-Laplacians and let $K_{\Delta_{p}^{v}[M(Y)]}$ be their integral kernels. Then the kernel $K_{\Delta_{p}(v)}$ can be obtained by the parametrix method gluing together the restriction of the kernel $K_{\Delta_{p}^{v}[M(Y)]}$ to $X(Y)$ and the heat kernel $K_{\Delta_{p}}$ of $X$ with respect to the fixed metric $g$ in exactly the same way as in section 13. The kernels $K_{\Delta_{p}^{v}[M(Y)]}$ depend smoothly on $v$, see [RS, Proposition 6.1] and [BGV, chapter 2.7], which applies to the present case, and thus, using exactly the same arguments as in the proof of Proposition [14.1 one can conclude (15.1).

Next, $(d / d v) \operatorname{Tr}_{\mathrm{rel}, u}\left(e^{-t \Delta_{p}(v)}\right)$ has a short time asymptotic expansion which arises by integrating the pointwise asymptotic expansion of $\operatorname{Tr}\left(\left((d / d v) \Delta_{p}(v)\right) K_{\Delta_{p}(v)}(t, x, x)\right)$ over $X$ resp. the support of $(d / d v) \Delta_{p}(v)$, which is compact. Thus, since $X$ is odd-dimensional, in the short time asymptotic expansion of $\operatorname{Tr}\left(\left((d / d v) \Delta_{p}(v)\right) K_{\Delta_{p}(v)}(t, x, x)\right)$ no constant term appears Gi]. As above, since $X$ with the metric $g_{v}$ is complete the operators $\Delta_{p}(v)$ are essentially selfadjoint [Ch] and thus the De Rham complex of $E_{\rho}$ valued differential forms has a unique ideal boundary condition whose cohomology equals the corresponding $L^{2}$-cohomology of $X$ with coefficients in $E_{\rho}$, [BL, Lemma 3.3, Theorem 3.5]. Since all $g_{v}$ are quasi isometric to $g$, this cohomology is zero by Proposition 8.1. Therefore, one has $\operatorname{Ker}\left(\Delta_{p}(v)\right)=0$ for all $v$ by the strong Hodge decomposition, see Proposition 12.1. Thus by Proposition 10.10, $\operatorname{Tr}_{\mathrm{rel}, u}\left(e^{-t \Delta_{p}(v)}\right)$ is exponentially decaying as $t \rightarrow \infty$ for each $v$.

Now one can establish the invariance of the relative analytic torsion under compact pertrubations of $g$ by inserting the arguments of the proof of [RS, Theorem 2.1]. For a compactly supported variation $h_{v}$ of the fibre metric $h$ on $E_{\rho}$ one proceeds analogously, taking the derivative of the corresponding operator $\#_{v}$, which varies with the fibre-metric, see [M̈̈4, section 2].

We will also need a gluing formula for the regularized analytic torsion on the manifolds $F_{X}(Y), Y>0$. Thus let $Y_{2}>Y$ and consider the manifold $F_{X}\left(Y_{2}\right) \subset F_{X}(Y)$. For

$$
Z_{X}\left[Y, Y_{2}\right]:=F_{X}(Y) \backslash F_{X}\left(Y_{2}\right)
$$


one has $F_{X}(Y)=Z_{X}\left[Y, Y_{2}\right] \sqcup_{\partial F_{X}\left(Y_{2}\right)} F_{X}\left(Y_{2}\right)$. The boundary of $Z_{X}\left[Y, Y_{2}\right]$ is the disjoint

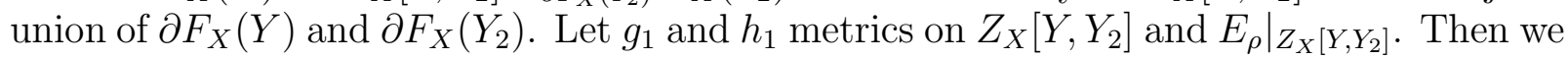
denote by $T\left(Z_{X}\left[Y, Y_{2}\right], \partial F_{X}(Y), g_{1}, h_{1} ; E_{\rho}\right)$ the analytic torsion of $Z_{X}\left[Y, Y_{2}\right]$ with coefficients in $E_{\rho}$ with relative boundary conditions at $\partial F_{X}(Y)$ and with absolute boundary conditions at $\partial F_{X}\left(Y_{2}\right)$ with respect to the metrics $g_{1}$ and $h_{1}$. If $g_{1}$ and $h_{1}$ are metrics on $F_{X}(Y)$ resp. $\left.E_{\rho}\right|_{F_{X}(Y)}$ which are compact perturbations of $g$ and $h$ and which are of product structure in a neighbourhood of $\partial F_{X}\left(Y_{2}\right)$, then as in (12.2) one has a short exact sequence of Hilbert complexes

$$
0 \rightarrow \mathcal{D}\left(F_{X}\left(Y_{2}\right), \partial F_{X}\left(Y_{2}\right) ; E_{\rho}\right) \rightarrow \mathcal{D}\left(F_{X}(Y), \partial F_{X}(Y) ; E_{\rho}\right) \rightarrow \mathcal{D}\left(Z\left[Y, Y_{2}\right], \partial F_{X}(Y) ; E_{\rho}\right) \rightarrow 0 .
$$

Since the cohomology groups $H^{*}\left(Z_{X}\left[Y, Y_{2}\right], \partial F(Y) ; E_{\rho}\right)$ are trivial, one obtains isomorphisms

$$
e_{p}\left(Y, Y_{2} ; E_{\rho}\right): H_{\min }^{p}\left(F_{X}\left(Y_{2}\right), \partial F_{X}\left(Y_{2}\right) ; E_{\rho}\right) \rightarrow H_{\min }^{p}\left(F_{X}(Y), \partial F_{X}(Y) ; E_{\rho}\right),
$$

which are defined by extension by zero. We recall that the cohomology groups in (15.2) are finite-dimensional and are isomorphic to the kernels of the corresponding Laplacians. Moreover, by the definition of the minimal extension, these cohomology groups depend only on the quasi-isometry class of $g_{1}$ and $h_{1}$ and are in particular invariant under compact perturbations of the metrics. Let $E_{p}\left(Y, Y_{2}, g_{1}, h_{1} ; E_{\rho}\right)$ be the matrix representing $e_{p}\left(Y, Y_{2} ; E_{\rho}\right)$ with respect to bases in the cohomology coming from $L^{2}$-orthonormal bases of harmonic forms associated to the metrics $g_{1}$ and $h_{1}$. Then the following gluing formula holds.

Proposition 15.2. Let $Y>0, Y_{2}>Y$. Let $g_{1}$ and $h_{1}$ be metrics on $F_{X}(Y)$ resp. $\left.E_{\rho}\right|_{F_{X}(Y)}$ which are compact perturbations of the metrics $g$ and $h$ and which are of product structure in a neighbourhood of $\partial F_{X}\left(Y_{2}\right)$ in $F_{X}(Y)$. Then one has

$$
\begin{aligned}
& \log T_{\mathrm{rel}, u}\left(F_{X}(Y), \partial F_{X}(Y), g_{1}, h_{1} ; E_{\rho}\right)=\log T_{\mathrm{rel}, u}\left(F_{X}\left(Y_{2}\right), \partial F_{X}\left(Y_{2}\right), g_{1}, h_{1} ; E_{\rho}\right) \\
+ & \log T\left(Z_{X}\left[Y, Y_{2}\right], \partial F(Y), g_{1}, h_{1} ; E_{\rho}\right)+\sum_{p}(-1)^{p} \log \left|\operatorname{det} E_{p}\left(Y, Y_{2}, g_{1}, h_{1} ; E_{\rho}\right)\right| .
\end{aligned}
$$

Proof. For the proof one can proceed exactly in the the same way as in the proof of Theorem 14.7.

Now we want to pass from the metrics $g_{1}$ on $F_{X}\left(Y_{1}\right)$ and $h_{1}$ on $\left.E_{\rho}\right|_{F_{X}\left(Y_{1}\right)}$ in Theorem 14.7 to the original metrics $g$ and $h$. For this purpose, we will apply the anomaly formula of Brüning an Ma [BM1], BM2. In the unimodular case, this formula is local on the boundary, and therefore, using the gluing formula from Proposition 15.2, we will be able to apply it also in the present non-compact stetting. We firstly recall the formula of Brüning and $\mathrm{Ma}$ for the very special case in which we will need it. For a metric $g_{1}$ on $Z_{X}\left[Y, Y_{2}\right]$ we let $B\left(\nabla_{g_{1}}^{T Z_{X}\left[Y, Y_{2}\right]}\right)$ be the $d-1$-form on $\partial Z_{X}\left[Y, Y_{2}\right]$ defined by Brüning and Ma in [BM1, (1.19)]. The form of [BM1, (1.19)] depends only on the Riemannian structure of an arbitrary neighbourhood of the boundary. Therefore, for a metric $g_{1}$ on $F_{X}(Y)$, one can also define a $d-1$-form $B\left(\nabla_{g_{1}}^{T F_{X}(Y)}\right)$ on $\partial F_{X}(Y)$ as in [BM1, (1.19)], although $F_{X}(Y)$ is not compact. Our flat bundle is unimodular and the boundaries we work with are always flat tori. Therefore, as already remarked in section 9, only the term involving the form 
[BM1, (1.19)] of the Brüning Ma anomaly formulas [BM1, Theorem 0.1], [BM2, Theorem $0.1]$ is present in our situations and the next proposition can thus be seen as its analog for the relative analytic torsion of the cusp.

Proposition 15.3. Let $Y>0$. Let $g_{1}$ and $h_{1}$ be metrics on $F_{X}(Y)$ and $\left.E_{\rho}\right|_{F_{X}(Y)}$ which are compact perturbations of the metrics $g$ and $h$ and which are of product structure in $a$ neighbourhood of $\partial F_{X}(Y)$. Let $B_{p}\left(Y ; g_{1}, h_{1}\right)$ be the matrix which represents the base change on $H_{\min }^{p}\left(F_{X}(Y), \partial F_{X}(Y)\right)$ from an orthonormal basis of harmonic forms with respect to the metrics $g$ and $h$ to an orthonormal basis of harmonic forms with respect to the metrics $g_{1}$ and $h_{1}$. Then one has

$$
\begin{aligned}
& \log T_{\mathrm{rel}, u}\left(F_{X}(Y), \partial F_{X}(Y), g_{1}, h_{1} ; E_{\rho}\right)=\log T_{\mathrm{rel}, u}\left(F_{X}(Y), \partial F_{X}(Y), g, h ; E_{\rho}\right) \\
- & \frac{1}{2} \operatorname{rk}\left(E_{\rho}\right) \int_{\partial F_{X}(Y)} B\left(\nabla_{g}^{T F_{X}(Y)}\right)+\sum_{p}(-1)^{p} \log \left|\operatorname{det} B_{p}\left(Y ; g_{1}, h_{1} ; E_{\rho}\right)\right| .
\end{aligned}
$$

Proof. We let $Y_{2}>Y$ and consider the manifold $F_{X}\left(Y_{2}\right) \subset F_{X}(Y)$. Assume that the metrics $g_{1}$ and $h_{1}$ are of product structure in a neighbourhood of $\partial F_{X}\left(Y_{2}\right)$ in $F_{X}(Y)$. Now we let $g_{2}$ and $h_{2}$ be metrics on $F_{X}(Y)$ resp. $\left.E_{\rho}\right|_{F_{X}(Y)}$ which coincide with the metrics $g$ and $h$ in a neighbourhood of $\partial F_{X}(Y)$ and which coincide with the metrics $g_{1}$ and $h_{1}$ in a neighbourhood of $F_{X}\left(Y_{2}\right)$ in $F_{X}(Y)$. If we apply Proposition 15.2 first for the metrics $g_{1}$ and $h_{1}$ and then for the metrics $g_{2}$ and $h_{2}$ and then subtract the first obtained equality from the second one and use that $g_{1}=g_{2}, h_{1}=h_{2}$ on $F_{X}\left(Y_{2}\right)$, we obtain:

$$
\begin{aligned}
& \log T_{\mathrm{rel}, u}\left(F_{X}(Y), \partial F_{X}(Y), g_{2}, h_{2} ; E_{\rho}\right)-\log T_{\mathrm{rel}, u}\left(F_{X}(Y), \partial F_{X}(Y), g_{1}, h_{1} ; E_{\rho}\right) \\
= & \log T\left(Z_{X}\left[Y, Y_{2}\right], \partial F_{X}(Y), g_{2}, h_{2} ; E_{\rho}\right)-\log T\left(Z_{X}\left[Y, Y_{2}\right], \partial F_{X}(Y), g_{1}, h_{1} ; E_{\rho}\right) \\
& +\sum_{p}(-1)^{p} \log \left|\operatorname{det} E_{p}\left(Y, Y_{2}, g_{2}, h_{2} ; E_{\rho}\right)\right|-\sum_{p}(-1)^{p} \log \left|\operatorname{det} E_{p}\left(Y, Y_{2}, g_{1}, h_{1} ; E_{\rho}\right)\right| .
\end{aligned}
$$

The metric $g_{1}$ is of product structure in a neighbourhood of $\partial Z_{X}\left[Y, Y_{2}\right]$ and the metric $g_{2}$ is of product structure in a neighbourhood of the component $\partial F_{X}\left(Y_{2}\right)$ of $\partial Z_{X}\left[Y, Y_{2}\right]$ and it equals the hyperbolic metric $g$ in a neighbourhoud of the component $\partial F_{X}(Y)$ of $\partial Z_{X}\left[Y, Y_{2}\right]$. Thus, since the cohomology $H^{*}\left(Z_{X}\left[Y, Y_{2}\right], \partial F_{X}(Y) ; E_{\rho}\right)$ is trivial and since the boundary $\partial Z\left[Y, Y_{2}\right]$ with its induced metric is a disjoint union of flat tori, it follows from [BM2, Theorem 3.4, (3.26)] and the local definition of the form in the second line of [BM2, (3.7)] that

$$
\begin{aligned}
& \log T\left(Z_{X}\left[Y, Y_{2}\right], \partial F_{X}(Y), g_{1}, h_{1} ; E_{\rho}\right) \\
= & \log T\left(Z_{X}\left[Y, Y_{2}\right], \partial F_{X}(Y), g_{2}, h_{2} ; E_{\rho}\right)-\frac{1}{2} \operatorname{rk}\left(E_{\rho}\right) \int_{\partial F_{X}(Y)} B\left(\nabla_{g}^{T F_{X}(Y)}\right) .
\end{aligned}
$$

We let $B_{p}\left(Y, g_{2}, h_{2} ; E_{\rho}\right)$ be the matrix which represents the change of bases on the space $H_{\min }^{p}\left(F_{X}(Y), \partial F_{X}(Y) ; E_{\rho}\right)$ from the basis of harmonic forms with respect to the metrics $g$ and $h$ to the basis of harmonic forms with respect to $g_{2}$ and $h_{2}$. Then we claim that, since 
$g_{2}$ and $h_{2}$ coincide with $g$ and $h$ in a neighbourhood of $\partial F_{X}(Y)$, one has

$$
\begin{aligned}
\log T_{\mathrm{rel}, u}\left(F_{X}(Y), \partial F_{X}(Y), g_{2}, h_{2} ; E_{\rho}\right)= & \log T_{\text {rel }, u}\left(F_{X}(Y), \partial F_{X}(Y), g, h ; E_{\rho}\right) \\
& +\sum_{p}(-1)^{p} \log \left|\operatorname{det} B_{p}\left(Y, g_{2}, h_{2} ; E_{\rho}\right)\right| .
\end{aligned}
$$

This equality woud again be well known for the situation of compact odd-dimensional manifolds with boundary, unitary resp. unimodular flat bundles and interior variations of the metric, [C1], BM2]. To extend it to the present case, one can proceed exactly as in the proof of Proposition 15.1 and we shall outline the main steps only: Firstly, one joins $g$ and $g_{2}, h$ and $h_{2}$ by a smooth path of metrics $g_{v}, h_{v} v \in[0,1]$ such that all metrics $g_{v}$ resp. $h_{v}$ coincide with $g$ and $h$ in a neighbourhood of the boundary and outside a fixed compact subset of $F_{X}(Y)$. Then, since the variation is compactly supported in the interior of $F_{X}(Y)$ and since $F_{X}(Y)$ is odd-dimensional, the short-time asymptotic expansion of $\operatorname{Tr}\left(\left((d / d v) \Delta_{q}^{v}\right) e^{-t \Delta_{q}^{v}}\right)$ has no constant term [Gi]. Therefore one can proceed exactly as in the closed case, [RS, Theorem 2.1, Proposition 6.1], [Mü1, Theorem 7.6] [Mü5, Theorem 2.6] and as in the proof of Proposition 15.1 to obtain

$$
\begin{aligned}
& \frac{d}{d v} \log T_{\mathrm{rel}, u}\left(F_{X}(Y), \partial F_{X}(Y), g_{v}, h_{v} ; E_{\rho}\right) \\
& =\frac{1}{2} \sum_{p}(-1)^{p} \operatorname{Tr}\left(\left(*_{v}^{-1} \frac{d}{d v} *_{v}+\#_{v}^{-1} \frac{d}{d v} \#_{v}\right) H_{v}^{p}\right),
\end{aligned}
$$

where $H_{v}^{p}$ is the orthogonal projection onto the space of harmonic forms with respect to the metrics $g_{v}$ and $h_{v}$. The argument of Brüning an Ma given in the last two paragraphs of the proof of [BM1, Theorem 4.5] carries over to the present situation of a Fredholm complex and it follows that the right hand side of (15.6) is the $v$-derivative of the corresponding change of the volume element on the determinant line of the cohomology, see also [RS, Proposition 6.4 and page 206]. This establishes (15.5).

Combining (15.3), (15.4) and (15.5) we obtain

$$
\begin{aligned}
& \log T_{\mathrm{rel}, u}\left(F_{X}(Y), \partial F_{X}(Y), g, h ; E_{\rho}\right)=\log T_{\text {rel }, u}\left(F_{X}(Y), \partial F_{X}(Y), g_{1}, h_{1} ; E_{\rho}\right) \\
& +\sum_{p}(-1)^{p}\left(\log \left|\operatorname{det} E_{p}\left(Y, Y_{2}, g_{2}, h_{2} ; E_{\rho}\right)\right|-\log \left|\operatorname{det} E_{p}\left(Y, Y_{2}, g_{1}, h_{1} ; E_{\rho}\right)\right|\right. \\
& \left.-\log \left|\operatorname{det} B_{p}\left(Y, g_{2}, h_{2} ; E_{\rho}\right)\right|\right)+\frac{1}{2} \operatorname{rk}\left(E_{\rho}\right) \int_{\partial F_{X}(Y)} B\left(\nabla_{g}^{T F_{X}(Y)}\right) .
\end{aligned}
$$

Using that $g_{1}=g_{2}, h_{1}=h_{2}$ on $F_{X}\left(Y_{2}\right)$, it follows directly from the definitions that

$$
B_{p}\left(Y, g_{1}, h_{1} ; E_{\rho}\right)=E_{p}\left(Y, Y_{2}, g_{1}, h_{1} ; E_{\rho}\right) \circ\left(E_{p}\left(Y, Y_{2}, g_{2}, h_{2} ; E_{\rho}\right)\right)^{-1} \circ B_{p}\left(Y, g_{2}, h_{2} ; E_{\rho}\right)
$$

and the Proposition is proved.

The previous proposition implies the gluing formula for the cusps for the original metrics $g$ and $h$ which now contains the anomaly. We use the notation from the beginning of this section. 
Corollary 15.4. Let $Y>0, Y_{2}>Y$. Then one has

$$
\begin{aligned}
& \log T_{\mathrm{rel}, u}\left(F_{X}(Y), \partial F_{X}(Y), g, h ; E_{\rho}\right)-\operatorname{rk}\left(E_{\rho}\right) \int_{\partial F_{X}(Y)} B\left(\nabla_{g}^{T F_{X}(Y)}\right) \\
= & \log T_{\mathrm{rel}, u}\left(F_{X}\left(Y_{2}\right), \partial F_{X}\left(Y_{2}\right), g, h ; E_{\rho}\right)-\operatorname{rk}\left(E_{\rho}\right) \int_{\partial F_{X}\left(Y_{2}\right)} B\left(\nabla_{g}^{T F_{X}\left(Y_{2}\right)}\right) \\
& +\sum_{p}(-1)^{p} \log \left|\operatorname{det} E_{p}\left(Y, Y_{2}, g, h ; E_{\rho}\right)\right| .
\end{aligned}
$$

Proof. We firstly replace the metrics $g$ on $F_{X}(Y)$ and $h$ on $\left.E_{\rho}\right|_{F_{X}(Y)}$ by metrics $g_{1}$ and $h_{1}$ which are compact perturbations of $g$ and $h$ and which are of product structure in a neighbourhood of $\partial F_{X}\left(Y_{2}\right)$ in $F_{X}(Y)$ and which coincide with $g$ and $h$ in a neighbourhood of $\partial F_{X}(Y)$. The torsion on $F_{X}(Y)$ changes as in (15.5). Then we apply Proposition 15.2 for the metrics $g_{1}$ and $h_{1}$. Finally, we apply Proposition 15.3 for the manifold $F_{X}\left(Y_{2}\right)$ to replace the metrics $g_{1}$ and $h_{1}$ on $F_{X}\left(Y_{2}\right)$ by $g$ and $h$. We obtain

$$
\begin{aligned}
& \log T_{\mathrm{rel}, u}\left(F_{X}(Y), \partial F_{X}(Y), g, h ; E_{\rho}\right)=\log T_{\mathrm{rel}, u}\left(F_{X}\left(Y_{2}\right), \partial F_{X}\left(Y_{2}\right), g, h ; E_{\rho}\right) \\
& -\frac{1}{2} \operatorname{rk}\left(E_{\rho}\right) \int_{\partial F_{X}\left(Y_{2}\right)} B\left(\nabla_{g}^{T F_{X}\left(Y_{2}\right)}\right)+\sum_{p}(-1)^{p} \log \left|\operatorname{det} E_{p}\left(Y, Y_{2}, g, h ; E_{\rho}\right)\right| \\
& +\log T\left(Z_{X}\left[Y, Y_{2}\right], g_{1}, h_{1} ; E_{\rho}\right),
\end{aligned}
$$

where $E_{p}\left(Y, Y_{2}, g, h ; E_{\rho}\right)$ is the matrix representing the map (15.2) with respect to $L^{2}$ orthonormal bases of harmonic forms associated to the metrics $g$ and $h$. For the relative Reidemeister torsion of the zylinder one has $\log \tau\left(Z_{X}\left[Y, Y_{2}\right], \partial F_{X}(Y) ; E_{\rho}\right)=0$. For the case of orthogonal representations this was proved by Milnor [Mil, Lemma 7.5] and the proof carries over to the unimodular case. Alternatively, one can use the combinatorial gluing formula [Mil], [Lu], [BM2], [Le]: $\log \tau\left(Z_{X}\left[Y, Y_{2}\right], \partial F_{X}(Y) ; E_{\rho}\right)=\log \tau\left(Z_{X}[Y, Y+\right.$ $\left.\epsilon], \partial F_{X}(Y) ; E_{\rho}\right)+\log \tau\left(Z_{X}\left[Y+\epsilon, Y_{2}\right], \partial F_{X}(Y+\epsilon) ; E_{\rho}\right)=2 \log \tau\left(Z_{X}\left[Y, Y_{2}\right], \partial F_{X}(Y) ; E_{\rho}\right)$. Thus by [BM2, Theorem 0.1, Remark 1.8 (ii); Theorem 3.4, (3.26)] and the definition of $g_{1}$ one has

$$
\log T\left(Z_{X}\left[Y, Y_{2}\right], \partial F_{X}(Y), g_{1}, h_{1} ; E_{\rho}\right)=\frac{1}{2} \operatorname{rk}\left(E_{\rho}\right) \int_{\partial F_{X}(Y)} B\left(\nabla_{g}^{T F_{X}(Y)}\right) .
$$

and the corollary follows.

We remark that in the compact case gluing formulas of the previous type were established by Brüning and Ma [BM2, Theorem 0.3, Theorem 0.4].

Now we can study the formula in Theorem 14.7 further. The metrics $g_{1}$ and $h_{1}$ used in this theorem are of product structure in a neighbourhood of $\partial X\left(Y_{1}\right)$. Therefore, since $\chi\left(\partial X_{1}\right)=0$, it follows from the Cheeger-Müller Theorem for manifolds with boundary, which in the present situation is due to Brüning and Ma BM2, Theorem 0.1, Remark 1.8 (ii)], that the analytic torsion $T\left(X\left(Y_{1}\right), g_{1}, h_{1} ; E_{\rho}\right)$ equals the corresponding Reidemeister 
torsion $\tau\left(X\left(Y_{1}\right), g_{1}, h_{1} ; E_{\rho}\right)$, i.e. that

$$
T\left(X\left(Y_{1}\right), g_{1}, h_{1} ; E_{\rho}\right)=\tau\left(X\left(Y_{1}\right), g_{1}, h_{1} ; E_{\rho}\right)
$$

where we use from now on the notation of section 9, For the case of unitary representations of the fundamental group, the corresponding result would be due to Lück [Lu] and Vishik Vi.

From now on, we shall drop the metrics $g$ and $h$ from the notation since these are the metrics we finally want to work with. Thus we shall write for example $T\left(F_{X}\left(Y_{1}\right), \partial F_{X}\left(Y_{1}\right) ; E_{\rho}\right)$ for $T\left(F_{X}\left(Y_{1}\right), \partial F_{X}\left(Y_{1}\right), g, h ; E_{\rho}\right)$. Let $D_{p}\left(Y_{1} ; E_{\rho}\right)$ be the matrix which represents the connecting homomorphism $\delta_{p}^{Y_{1}}$ from (12.3) with respect to the basis of $H^{p}\left(X\left(Y_{1}\right) ; E_{\rho}\right)$ consisting of restrictions of Eisenstein series to $X\left(Y_{1}\right)$ as in the end of section 9 and to the orthonormal basis of harmonic forms in $H_{\min }^{p+1}\left(F_{X}\left(Y_{1}\right), \partial F_{X}\left(Y_{1}\right) ; E_{\rho}\right)$ with respect to the original metrics $g$ and $h$. Then combining (9.1), Theorem 14.7, Proposition 15.1, Proposition 15.3 and (15.7), we obtain:

$$
\begin{aligned}
\log T_{\mathrm{rel}, u}\left(X ; E_{\rho}\right) & =\log \tau_{E i s}\left(\bar{X} ; E_{\rho}\right)+\log T_{\mathrm{rel}, u}\left(F_{X}\left(Y_{1}\right), \partial F_{X}\left(Y_{1}\right) ; E_{\rho}\right) \\
& +\sum_{p}(-1)^{p} \log \left|\operatorname{det} D_{p}\left(Y_{1} ; E_{\rho}\right)\right|-\frac{1}{2} \operatorname{rk}\left(E_{\rho}\right) \int_{\partial F_{X}\left(Y_{1}\right)} B\left(\nabla_{g}^{T F_{X}(Y)}\right) .
\end{aligned}
$$

We next replace $F_{X}\left(Y_{1}\right)$ by $F_{X}$ using the gluing formula for the cusp from Corollary 15.4 , Here $F_{X}$ is $F_{X}(1)$ as in section 2.7. We can assume that $Y_{1} \geq 1$ in (15.8). Let $E_{p}\left(Y_{1} ; E_{\rho}\right)$ be the matrix which represents the extension by zero from $H_{\min }^{p}\left(F_{X}\left(Y_{1}\right), \partial F_{X}\left(Y_{1}\right) ; E_{\rho}\right)$ to $H_{\text {min }}^{p}\left(F_{X}, \partial F_{X} ; E_{\rho}\right)$ from (15.2) with respect to the inner products on the cohomologies induced by the harmonic forms associated to the metrics $g$ and $h$ on $F_{X}\left(Y_{1}\right),\left.E_{\rho}\right|_{F_{X}\left(Y_{1}\right)}$ resp. $F_{X},\left.E_{\rho}\right|_{F_{X}}$. Then from (15.8) and Corollary 15.4 we obtain

$$
\begin{aligned}
\log T_{\mathrm{rel}, u}\left(X ; E_{\rho}\right) & =\log \tau_{\text {Eis }}\left(\bar{X} ; E_{\rho}\right)+\log T_{\mathrm{rel}, u}\left(F_{X}, \partial F_{X} ; E_{\rho}\right)-\frac{1}{2} \operatorname{rk}\left(E_{\rho}\right) \int_{\partial F_{X}} B\left(\nabla_{g}^{T F_{X}}\right) \\
& +\sum_{p}(-1)^{p}\left(\log \left|\operatorname{det} D_{p}\left(Y_{1} ; E_{\rho}\right)\right|-\log \left|\operatorname{det} E_{p}\left(Y_{1} ; E_{\rho}\right)\right|\right) .
\end{aligned}
$$

Now we use again results of Brüning an Ma who for the present case computed the anomaly explicitly in BM1, section 4.5]. We realize a neighbourhood of $\partial F_{X}$ in $F_{X}$ as $\partial F_{X} \times[0, \epsilon)$; the hyperbolic metric $g$ is then given by $g\left(w, x_{d}\right)=\left(1+x_{d}\right)^{-2}\left(d x_{d}^{2}+g_{\partial F_{X}}\right)$, $w \in \partial F_{X}, x_{d} \in[0, \epsilon)$ and where $g_{\partial F_{X}}$ denotes the restriction of $g$ to $\partial F_{X}$. We may assume that the restrictions of the metrics $g$ and $g_{1}$ to $\partial F_{X}$ coincide. According to [BM1, (4.38)], for $f(v):=(1+v)^{2}$ we let $g_{s}\left(v, x_{d}\right):=f\left(s x_{d}\right) g$, which is is a smooth path between $g$ and the metric $g_{1}$ on $\partial F_{X} \times[0, \epsilon)$ for $\epsilon$ sufficiently small. Since $\partial F_{X}$ with the hyperbolic metric is a union of finitely many disjoint flat tori, by [BM1, (4.42)], [BM1, Theorem 0.1, Theorem $4.5,(4.7)]$ for

$$
c(n):=\frac{(-1)^{n}(2 n-1) !}{2^{2 n+1} \pi^{n} n !}
$$


one has

$$
\frac{1}{2} \int_{\partial F_{X}} B\left(\nabla_{g}^{T F_{X}}\right)=\frac{(-1)^{n}(2 n) !}{2^{2 n+1} \pi^{n} n !} \operatorname{vol}\left(\partial F_{X}\right) \int_{0}^{1} s^{2 n-1} d s=c(n) \operatorname{vol}\left(\partial F_{X}\right) .
$$

Here, as above we may replace $F_{X}$ by the manifold $Z_{X}[1,2]$ with suitable metrics in order to apply [BM1, Theorem 4.5, (4.7)].

We remark that the left hand side in (15.9) does not depend on $Y_{1}$ anymore and we will see below that the second line in (15.9) doesn't depend on $Y_{1}$ either.

\section{The COntribution of The COHOMOlogy to The Gluing FORMula}

We keep the notations of the previous section. In order to complete the proof of our main result, it remains to compute the contribution of the cohomology, i.e. the determinants in the second line of (15.9).

To begin with, we recall that if $\omega$ is a smooth $E_{\rho}$-valued $p$-form on $F_{X}(Y)$ which is squareintegrable, for which also $d \omega$ is square-integrable and which restricts to zero on $\partial F_{X}(Y)$, then $\omega$ belongs to $\mathcal{D}_{\min }^{*}\left(F_{X}(Y), \partial F_{X}(Y) ; E_{\rho}\right)$. This can be proved proceeding exactly as in the proof of the corresponding statement by Brüning in Lesch [BL, Theorem 4.1] for compact manifolds with boundary. Here we remark that to deal with the non-compact end of $F_{X}(Y)$ one just takes a sequence $\phi_{n}$ of smooth compactly supported functions which are idenitcally 1 on $F_{X}(Y+n)$, which are bounded and whose derivative is bounded. Such a sequence obviously exists in the present case. Thus, using the methods of section 6, one can now construct explicitly an orthonormal basis as follows. We equip the spaces $\mathcal{H}^{k}\left(\mathfrak{n}_{P_{j}} ; V_{\rho}\right)$ with the inner product defined in the last paragraph of section 9. Then we have the following proposition.

Proposition 16.1. Let $Y>0$. Let $\rho \in \hat{G}, \rho \neq \rho_{\theta}$. Let $k>n$. For each $P_{j} \in \mathfrak{P}_{\Gamma}$ let $\Phi_{i, j}^{k}$, $i=1, \ldots, \operatorname{dim} \mathcal{H}^{k}\left(\mathfrak{n}_{P_{j}} ; V_{\rho}\right)$, be an orthonormal basis of $\mathcal{H}^{k}\left(\mathfrak{n}_{P_{j}} ; V_{\rho}\right)$. Then an orthonormal basis of $H_{\min }^{k+1}\left(F_{X}(Y), \partial F_{X}(Y) ; E_{\rho}\right)$ is given by the cohomology classes of the sections

$$
\sqrt{2\left|\lambda_{\rho, k}\right|} \cdot Y^{-\lambda_{\rho, k}} \cdot\left(\widetilde{\Phi_{i, j}^{k}}\right)_{\lambda_{\rho, k}}
$$

where $\left(\widetilde{\Phi_{i, j}^{k}}\right)_{\lambda_{, k}}$ is as in ([6.9). Let $\Phi_{i, j}^{n}, i=1, \ldots, \operatorname{dim} \mathcal{H}^{n}\left(\mathfrak{n}_{P_{j}} ; V_{\rho}\right)_{-}$, be an orthonormal basis of $\mathcal{H}^{n}\left(\mathfrak{n}_{P_{j}} ; V_{\rho}\right)_{-}$. Then an orthonormal basis of $H_{\min }^{n+1}\left(F_{X}(Y), \partial F_{X}(Y) ; E_{\rho}\right)$ is given by the cohomology classes of the sections

$$
\sqrt{2\left|\lambda_{\rho, n}^{-}\right|} \cdot Y^{-\lambda_{\rho, n}^{-}} \cdot\left(\widetilde{\Phi_{i, j}^{n}}\right)_{\lambda_{\rho, n}^{-}}
$$

Proof. By Lemma 6.3, the forms in the proposition are harmonic and restrict to zero on $\partial F_{X}(Y)$. By the assumption on $\rho$, one has $\lambda_{\rho, n}^{-}<0$ and $\lambda_{\rho, k}<0$ for $k>n$. Thus by (6.10) they form an orthonormal system in $L^{2}\left(F_{X}(Y) ; \Lambda^{k+1} E_{\rho}\right)$. Using Proposition 8.5, Proposition 8.7 Proposition 12.2 and the preceding remarks it follows that the corresponding cohomology classes form a basis of $H_{\min }^{k+1}\left(F_{X}(Y), \partial F_{X}(Y) ; E_{\rho}\right)$. 
Using the preceding proposition, the isomorphism (8.1) and the fact that $\operatorname{dim} \mathcal{H}^{n}\left(\mathfrak{n}_{P_{j}} ; V_{\rho}\right)=$ $2 \operatorname{dim} \mathcal{H}^{n}\left(\mathfrak{n}_{P_{j}} ; V_{\rho}\right)_{-}$, we can immediately compute the term $\operatorname{det} E_{p}\left(Y_{1} ; E_{\rho}\right)$ which appears in the second line of (15.9):

Corollary 16.2. Let $Y_{1}>1$. Then for $k>n$ one has

$$
\operatorname{det} E_{k+1}\left(Y_{1} ; E_{\rho}\right)=Y_{1}^{\lambda_{\rho, k} \operatorname{dim} H^{k}\left(\partial \bar{X} ; E_{\rho}\right)} \text {. }
$$

For $k=n$ one has

$$
\operatorname{det} E_{k+1}\left(Y_{1} ; E_{\rho}\right)=Y_{1}^{\lambda_{\rho, k} \operatorname{dim} H^{n}\left(\partial \bar{X} ; E_{\rho}\right) / 2} .
$$

Proof. This follows immediately from Proposition 16.1 and (6.10).

We now compute the determinant of $D_{p}\left(Y_{1} ; E_{\rho}\right)$, which represents the matrix $\delta_{p}\left(Y_{1}\right)$ in the same way as in the previous section. By construction, the map $\delta_{p}\left(Y_{1}\right)$ is defined as follows. Let $[f] \in H^{p}\left(X\left(Y_{1}\right) ; E_{\rho}\right)$ be a cohomology class, where $f$ is a smooth section of $E_{\rho}$ which is closed. Let $\tilde{f}$ be an extension of $f$ to a smooth, square-integrable section of $E_{\rho}$ on $X$ such that also $d \tilde{f}$ is square-integrable. Then one has $\delta_{p}\left(Y_{1}\right)([f])=\left[d\left(\left.\tilde{f}\right|_{F_{X}\left(Y_{1}\right)}\right)\right]$. If $h$ is any smooth section of $E_{\rho}$ over $F_{X}\left(Y_{1}\right)$ which is square-integrable and for which $d h$ is square-integrable and which coincides with $f$ on $\partial F_{X}\left(Y_{1}\right)$, then by the above remarks $h-\tilde{f} \in \mathcal{D}^{p}\left(F_{X}\left(Y_{1}\right), \partial F_{X}\left(Y_{1}\right) ; E_{\rho}\right)$. Thus one also has $\delta_{p}\left(Y_{1}\right)([f])=[d h]$.

Proposition 16.3. For each $k=n+1, \ldots, 2 n$ one has

$$
\operatorname{det}\left(D_{k}\left(Y_{1} ; E_{\rho}\right)=\left(\sqrt{2\left|\lambda_{\rho, k}\right|} \cdot Y_{1}^{-\lambda_{\rho, k}}\right)^{\operatorname{dim} H^{k}\left(\partial \bar{X} ; E_{\rho}\right)} .\right.
$$

For $k=n$ one has

$$
\operatorname{det}\left(D_{n}\left(Y_{1}: E_{\rho}\right)\right)=\left(\sqrt{2\left|\lambda_{\rho, n}^{-}\right|} \cdot Y_{1}^{-\lambda_{\rho, n}^{-}}\right)^{\operatorname{dim} H^{n}\left(\partial \bar{X} ; E_{\rho}\right) / 2} .
$$

Proof. First assume that $k>n$. Fix $\Phi \in \mathcal{H}^{k}\left(\mathfrak{n}_{P_{j}} ; V_{\rho}\right)$. Since $\lambda_{\rho, k}<0$, the forms $\Phi_{\lambda_{\rho, k}}$ and $\tilde{\Phi}_{\lambda_{\rho, k}}$ belong to $L^{2}\left(F_{X}\left(Y_{1}\right), \nu_{p}(\rho)\right) \cong L^{2}\left(F_{X}\left(Y_{1}\right), \Lambda^{p} E_{\rho}\right)$ resp. to $L^{2}\left(F_{X}\left(Y_{1}\right), \nu_{p+1}(\rho)\right) \cong$ $L^{2}\left(F_{X}\left(Y_{1}\right), \Lambda^{p+1} E_{\rho}\right)$. By Lemma 5.1 and equation (6.4) we have

$$
d\left(\Phi_{\lambda_{\rho, k}}\right)=2 \lambda_{\rho, k} \tilde{\Phi}_{\lambda_{\rho, k}} .
$$

By Lemma 5.2, equation (6.4) and Proposition 8.5, the restrictions of $Y_{1}^{-2 \lambda_{\rho, k}} \cdot \Phi_{\lambda_{\rho, k}}$ and $E\left(\Phi:-\lambda_{\rho, k}\right)$ to $\partial X\left(Y_{1}\right)$ are cohomologous. Thus by the above remarks one has

$$
\delta_{k}\left(Y_{1}\right)\left(E\left(\Phi:-\lambda_{\rho, k}\right)\right)=d\left(Y_{1}^{-2 \lambda_{\rho, k}} \Phi_{\lambda_{\rho, k}}\right) .
$$

Applying (16.1) and Proposition 16.1, the Proposition follows for $k>n$.

It remains to consider the case $k=n$. Fix $\Phi \in \mathcal{H}^{n}\left(\mathfrak{n}_{P_{j}} ; V_{\rho}\right)$. By Proposition 8.7, for $P_{l} \in \mathfrak{P}_{\Gamma}$ the restriction of $E\left(\Phi:-\lambda_{\rho, n}^{-}\right)$to the boundary component $\partial X\left(Y_{1}\right)_{P_{l}}$ of $X\left(Y_{1}\right)$ associated to $P_{l}$ is cohomologous to

$$
\delta_{j, l} \Phi+\left[\underline{C}_{P_{j} \mid P_{l}}\left(\sigma_{\rho, n}^{-}:-\lambda_{\rho, n}^{-}\right) \Phi\right]_{+} .
$$


Here one has $\left[\underline{C}_{P_{j} \mid P_{l}}\left(\sigma_{\rho, n}:-\lambda_{\rho, n}^{-}\right) \Phi\right]_{+} \in \mathcal{H}^{n}\left(\mathfrak{n}_{P_{l}}, V_{\rho}\right)_{+}$. Thus, since $\lambda_{\rho, n}^{+}=-\lambda_{\rho, n}^{-}$, by Lemma 5.2 and equation (6.4) the form

$$
\left(\left[\underline{C}_{P_{j} \mid P_{l}}\left(\sigma_{\rho, n}:-\lambda_{\rho, n}^{-}\right) \Phi\right]_{+}\right)_{\lambda_{\rho, n}^{-}},
$$

defined as in (5.2), restricts to $\left[\underline{C}_{P_{j} \mid P_{l}}\left(\sigma_{\rho, n}:-\lambda_{\rho, n}^{-}\right) \Phi\right]_{+}$on $\partial F_{P_{l}}\left(Y_{1}\right)$. However, since $\lambda_{\rho, n}^{-}<0$, the form in (16.2) is square integrable on $F_{P_{l}}\left(Y_{1}\right)$ and by Lemma 5.1 it is closed. Therefore, exactly the same argument as before gives

$$
\delta_{n}\left(Y_{1}\right)\left(E\left(\Phi:-\lambda_{\rho, n}^{-}\right)\right)=d\left(Y_{1}^{-2 \lambda_{\rho, n}^{-}} \Phi_{\lambda_{\rho, n}^{-}}\right)
$$

and applying (16.1) and Proposition 16.1, the Proposition follows also for $k=n$.

Now we remark that by the Proposition 8.6 and by Proposition 12.2 the last line in (15.9) can be written as

$$
\sum_{k=n}^{2 n}(-1)^{k}\left(\log \left|\operatorname{det} D_{k}\left(Y_{1} ; E_{\rho}\right)\right|+\log \left|\operatorname{det} E_{k+1}\left(Y_{1} ; E_{\rho}\right)\right|\right) .
$$

Thus it equals

$$
\frac{(-1)^{n}}{4} \log \left(2\left|\lambda_{\rho, n}^{-}\right|\right) \cdot \operatorname{dim} H^{n}\left(\partial \bar{X} ; E_{\rho}\right)+\sum_{k=n+1}^{2 n} \frac{(-1)^{k}}{2} \log \left(2\left|\lambda_{\rho, k}\right|\right) \cdot \operatorname{dim} H^{k}\left(\partial \bar{X} ; E_{\rho}\right)
$$

Now we use that $\chi(\partial \bar{X})=0$. Then we use that that $\operatorname{dim} H^{k}\left(\partial \bar{X} ; E_{\rho}\right)=\operatorname{dim} H^{2 n-k}\left(\partial \bar{X} ; E_{\rho}\right)$ by the computations of section 6 and by (8.1), although $E_{\rho}$ may not be self-dual, and that $\lambda_{\rho, k}=-\lambda_{\rho, 2 n-k}$ for $k \neq n$ and that $\left|\lambda_{\rho, n}^{-}\right|=\left|\lambda_{\rho, n}\right|$. Then (16.3) becomes

$$
\frac{1}{4} \sum_{k=0}^{2 n}(-1)^{k} \log \left(\left|\lambda_{\rho, k}\right|\right) \cdot \operatorname{dim} H^{k}\left(\partial \bar{X} ; E_{\rho}\right)
$$

and Theorem 1.1 is established. Here we remark that by the remarks from the end of section 10, we may pass from the relative to the regularized traces in (15.9).

\section{REFERENCES}

[BV] N. Bergeron, A. Venkatesh, The asymptotic growth of torsion homology for arithmetic groups, J. Inst. Math. Jussieu 12 (2013), no. 2, 391-447

[BGV] N. Berline, E. Getzler, M. Vergne, Heat kernels and Dirac operators, Corrected reprint of the 1992 original. Grundlehren Text Editions. Springer-Verlag, Berlin, 2004

[BGM] M. Berger, P. Gauduchon, E. Mazet, Le spectre d'une varieté Riemannienne, Lecture Notes in Mathematics 194. Berlin, Heidelberg, New York: Springer 1971

[BZ] J. Bismut, W. Zhang, An extension of a theorem by Cheeger and Müller. With an appendix by François Laudenbach, Astérisque No. 205 (1992)

[BMZ] J.M. Bismut, X. Ma, W. Zhang, Asymptotic torsion and Toeplitz operators, Preprint, 2011, available at http://www.math.jussieu.fr/ ma/mypubli/BismutMaZhangglob.pdf

[Bo1] A. Borel, Introduction aux groupes arithmétiques, Publications de l'Institut de Mathématique de l'Université de Strasbourg, XV. Actualités Scientifiques et Industrielles, No. 1341 Hermann, Paris 1969. 
[Bo2] A. Borel, Regularization theorems in Lie algebra cohomology. Applications, Duke Math. J. 50 (1983), no. 3, 605-623

[BC] A. Borel, W. Casselman, L2-cohomology of locally symmetric manifolds of finite volume, Duke Math. J. 50 (1983), no. 3, 625-647

[BG] A.Borel, H, Garland, Laplacian and the discrete spectrum of an arithmetic group, Amer. J. Math. 105 (1983), no. 2, 309-335.

[BW] A. Borel, N. Wallach, Continuous cohomology, discrete subgroups, and representations of reductive groups, Princeton University Press, Princeton, 1980

[BL] J. Brüning, M. Lesch, Hilbert complexes, J. Funct. Anal. 108 (1992), no. 1, 88-132.

[BM1] J. Brüning, X. Ma, An anomaly formula for Ray-Singer metrics on manifolds with boundary, Geom. Funct. Anal. 16 (2006), no. 4, 767-837.

[BM2] J. Brüning, X. Ma, On the gluing formula for the analytic torsion, Math. Z. 273 (2013), no. 3-4, 1085-1117

[CV] F. Calegari, A. Venkatesh, A torsion Jacquet-Langlands correspondence, Preprint 2012, arXiv:1212.3847

[C1] J. Cheeger, Analytic torsion and the heat equation, Ann. of Math. (2) 109 (1979), no. 2, 259-322

[C2] J. Cheeger, Spectral geometry of singular Riemannian spaces, J. Differential Geom. 18 (1983), no. 4, 575-657

[Ch] P. Chernoff, Essential self-adjointness of powers of generators of hyperbolic equations, J. Funct. An. 12 (1973), 401-414

[CJ] H. Carslaw, J. Jaeger, Conduction of heat in solids, Reprint of the second edition, Oxford Science Publications, The Clarendon Press, Oxford University Press, New York, 1988

[Do1] H. Donnelly, Spectral geometry for certain noncompact Riemannian manifolds, Math. Z. 169 (1979), no. 1, 63-76

[Do2] H. Donnelly, Asymptotic expansions for the compact quotients of properly discontinuous group actions, Illinois J. Math. 23 (1979), no. 3, 485-496.

[vE] W. van Est, A generalization of the Cartan-Leray spectral sequence. I, II, Nederl. Akad. Wetensch. Proc. Ser. A 61 = Indag. Math. 20 1958, 399-413

[Gi] P. Gilkey, Invariance theory, the heat equation, and the Atiyah-Singer index theorem, Second edition, Studies in Advanced Mathematics, CRC Press, Boca Raton, FL, 1995.

[GW] R. Goodman, N. Wallach, Representations and invariants of the classical groups, Encyclopedia of Mathematics and its Applications, 68. Cambridge University Press, Cambridge, 1998

[Gr] P. Greiner, An asymptotic expansion for the heat equation, Arch. Rational Mech. Anal. 41 (1971), 163-218.

[Ha] G. Harder, On the cohomology of discrete arithmetically defined groups, In: Discrete subgroups of Lie groups and applications to moduli (Internat. Colloq., Bombay, 1973), pp. 129-160. Oxford Univ. Press, Bombay, 1975

[Has] A. Hassell, Analytic surgery and analytic torsion, Comm. Anal. Geom. 6 (1998), no. 2, 255-289.

[HC] Harish-Chandra, Automorphic forms on semisimple Lie gropus, Springer-Verlag Lecture Notes in Mathematics 62 (1968)

[Kn1] A. Knapp, Representation theory of semisimple groups, Princeton University Press, Princeton, 2001.

[Kn2] A. Knapp, Lie Groups Beyond an introduction, Second Edition, Birkhäuser, Boston, 2002.

[Ko] B. Kostant, Lie algebra cohomology and the generalized Borel-Weil theorem, Annals of Math. (2) 74 (1961), 329-378

[KS] A. Knapp, E. Stein, Intertwining operators for semisimple Lie groups, Annals of Math (2) 93 (1971), 489-578

[La] R. Langlands, On the functional equations satisfied by Eisenstein series, Springer-Verlag Lecture Notes in Mathematics 544 (1976) 
[Le] M. Lesch, A gluing formula for the analytic torsion on singular spaces, Anal. PDE 6 (2013), no. 1, 221-256.

[LR] J. Lott, M. Rothenberg, Analytic torsion for group actions, J. Differential Geom. 34 (1991), no. $2,431-481$

[Lu] W. Lück, Analytic and topological torsion for manifolds with boundary and symmetry, J. Differential Geom. 37 (1993), no. 2, 263-322

[LS] W. Lück, T. Schick, L2-torsion of hyperbolic manifolds of finite volume, Geom. Funct. Anal. 9 (1999), no. 3, 518-567

[MM] S. Marshall, W. Müller, On the torsion in the cohomology of arithmetic hyperbolic 3-manifolds, Duke Math. J. 162 (2013), no. 5, 863-888.

[MaMu] Matsushima, Murakami, On vector bundle valued harmonic forms and automorphic forms on symmetric riemannian manifolds, Ann. of Math. (2) 78 (1963), 365-416

[Me] R.B. Melrose, The Atiyah-Patodi-Singer index theorem, Research Notes in Mathematics, 4. A K Peters, Ltd., Wellesley, MA, 1993.

[MePo] P. Menal-Ferrer, J. Porti, Higher dimensional Reidemeister torsion invariants for cusped hyperbolic 3-manifolds, to appear in Journal of Topology, DOI: 10.1112/jtopol/jtt024

[Mi1] R. J. Miatello, The Minakshisundaram-Pleijel coefficients for the vector-valued heat kernel on compact locally symmetric spaces of negative curvature. Trans. Amer. Math. Soc. 260 (1980), no. $1,1-33$.

[Mil] J. Milnor, Whitehead torsion, Bull. Amer. Math. Soc. $72(1966$,$) 358-426.$

[MS] H. Moscovici, R. Stanton, R-torsion and zeta functions for locally symmetric manifolds, Inv. Math. 105 (1991), 185-216,

[Mü1] W. Müller, Analytic torsion and R-torsion of Riemannian manifolds, Adv. in Math. 28 (1978), no. $3,233-305$

[Mü2] W. Müller, Spectral theory for Riemannian manifolds with cusps and a related trace formula, Math. Nachr. 111 (1983), 197-288.

[Mü3] W. Müller, Manifolds with cusps of rank one. Spectral theory and $L^{2}$-index theorem, SpringerVerlag Lecture Notes in Mathematics 1244 (1987)

[Mü4] W. Müller, Analytic torsion and R-torsion for unimodular representations, J. Amer. Math. Soc. 6 (1993), 721-753.

[Mü5] W. Müller, Relative zeta functions, relative determinants and scattering theory, Comm. Math. Phys. 192 (1998), no. 2, 309-347

[MP1] W. Müller, J. Pfaff, On the asymptotics of the Ray-Singer analytic torsion for compact hyperbolic manifolds, Intern. Math. Research Notices 2013, no. 13, 2945-2984.

[MP2] W. Müller, J. Pfaff, Analytic torsion of complete hyperbolic manifolds of finite volume, J. Funct. Anal. 263 (2012), no. 9, 2615-2675

[MP3] W. Müller, J. Pfaff, Analytic torsion and L2-torsion of compact locally symmetric manifolds, J. Differential Geom. 95 (2013), no. 1, 71-119.

[MP4] W. Müller, J. Pfaff, On the growth of torsion in the cohomology of arithmetic groups, Preprint 2013, arXiv:1302.0742

[MP5] W. Müller, J. Pfaff, The analytic torsion and its asymptotic behaviour for sequences of hyperbolic manifolds, Preprint 2013, arXiv:1307.4914

[MuS] W. Müller, G. Salomonsen, Scattering theory for the Laplacian on manifolds with bounded curvature, J. Funct. Anal. 253 (2007), no. 1, 158-206.

[Par] J. Park, Analytic torsion and Ruelle zeta functions for hyperbolic manifolds with cusps, J. Funct. An. 257 (2009), no. 6, 1713-1758

[Paq] L. Paquet, Problemes mixtes pour le systme de Maxwell, Ann. Fac. Sci. Toulouse Math. (5) 4 (1982), no. 2, 103-141

[Pf1] J. Pfaff, Selberg zeta functions on odd-dimensional hyperbolic manifolds of finite volume, to appear in J. Reine und Angewandte Mathematik, DOI: 10.1515/crelle-2013-0047 
[Pf2] J. Pfaff, Analytic torsion versus Reidemeister torsion on hyperbolic 3-manifolds with cusps, to appear in Mathematische Zeitschrift, arXiv:1206.0228

[Ra] J. Raimbault, Asymptotics of analytic torsion for hyperbolic three-manifolds, Preprint 2012, arXiv:1212.3161

[RS] D. Ray, I.M Singer, R-torsion and the Laplacian on Riemannian manifolds. Advances in Math 7, 145-210. (1971).

[Sch] J. Schwermer, Kohomologie arithmetisch definierter Gruppen und Eisensteinreihen, Lecture Notes in Mathematics 988, Springer-Verlag, Berlin, 1983

[Se] A. Selberg, Harmonic analysis, in "Collected Papers", Vol. I, Springer-Verlag, Berlin-HeidelbergNew York (1989), 626-674.

[Ta] M. Taylor, Pseudo-differential operators, Princeton University Press, Princeton, NJ, 1981.

[Ve] B. Vertman, Analytic torsion of a bounded generalized cone, Comm. Math. Phys. 290 (2009), no. 3, 813-860

[Vi] S. Vishik, Generalized Ray-Singer conjecture. I. A manifold with a smooth boundary, Comm. Math. Phys. 167 (1995), no. 1, 1-102

[Wa] G. Warner, Selberg's trace formula for nonuniform lattices: the R-rank one case, Studies in algebra and number theory, Adv. in Math. Suppl. Stud. 6, Academic Press, New York-London, 1979.

[Wa2] G. Warner, Harmonic analysis on semi-simple Lie groups, I, Die Grundlehren der mathematischen Wissenschaften, Band 188, Springer-Verlag, New York-Heidelberg, 1972

[Wh] G. W. Whitehead, Elements of homotopy theory, Graduate Texts in Mathematics, 61. SpringerVerlag, New York-Berlin, 1978.

Universität Bonn, Mathematisches Institut, Endenicher Alle 60, D - 53115 Bonn, GerMANY

E-mail address: pfaff@math.uni-bonn.de 